\title{
WestVirginiaUniversity
}

THE RESEARCH REPOSITORY @ WVU

Graduate Theses, Dissertations, and Problem Reports

2012

\section{Three Essays on Financial and Trade Integration}

\author{
Elena Bondarenko \\ West Virginia University
}

Follow this and additional works at: https://researchrepository.wvu.edu/etd

\section{Recommended Citation}

Bondarenko, Elena, "Three Essays on Financial and Trade Integration" (2012). Graduate Theses,

Dissertations, and Problem Reports. 150.

https://researchrepository.wvu.edu/etd/150

This Dissertation is protected by copyright and/or related rights. It has been brought to you by the The Research Repository @ WVU with permission from the rights-holder(s). You are free to use this Dissertation in any way that is permitted by the copyright and related rights legislation that applies to your use. For other uses you must obtain permission from the rights-holder(s) directly, unless additional rights are indicated by a Creative Commons license in the record and/ or on the work itself. This Dissertation has been accepted for inclusion in WVU Graduate Theses, Dissertations, and Problem Reports collection by an authorized administrator of The Research Repository @ WVU.

For more information, please contact researchrepository@mail.wvu.edu. 


\title{
Three Essays on Financial and Trade Integration
}

\author{
Elena Bondarenko \\ Dissertation submitted to the \\ College of Business and Economics \\ at West Virginia University \\ in partial fulfillment of the requirements \\ for the degree of \\ Doctor of Philosophy \\ in \\ Economics
Shuichiro Nishioka, Ph.D., Chair
Ronald Balvers, Ph.D.
Arabinda Basistha, Ph.D.
Stratford Douglas, Ph.D.
Alexander Kurov, Ph.D.
Department of Economics
Morgantown, West Virginia
2012

Keywords: Financial Capital Flows; Trade Openness; Capital Controls; Capital Content of Trade; Physical Capital; Marginal Product of Capital; Convergence; Economic Growth 


\section{Abstract \\ Three Essays on Financial and Trade Integration \\ Elena Bondarenko}

This dissertation consists of three essays on financial and trade integration. Financial and trade integration are the processes though which a country's financial and commodities markets become more integrated with those in other countries. The first essay addresses the determinants of financial integration, the second essay examines the contribution of financial and trade integration to the convergence in marginal products of capital, the third essay accesses the effect of international trade in physical capital on economic growth.

The first essay addresses the empirical question of whether international financial flows are responsive to capital account restrictions or liberalization policies. The effect of capital controls on financial flows differs across countries and types of financial flows (FDI, portfolio equity, and debt). Capital controls are found to be effective for all types of international capital flows in developed countries. However, short-term volatile flows are not responsive to capital controls in developing countries. Capital controls can be an effective policy tool in developed countries with liberalized international trade and adequate reserves. Policies in developing countries should facilitate FDI flows and restrict non-productive short-term equity or debt flows in order to maintain macroeconomic stability and lower the probability of a crisis.

The second essay examines the determinants of convergence in the marginal product of capital. The essays derives an empirical model from Solow's growth model and augment it to include global factors of financial flows and capital embodied in commodity trade. The marginal products of capital converges, however, this convergence is conditional upon country- specific variables such as reproducible capital share. Saving rates, foreign direct investment, and international trade are essential determinants of this conditional convergence. There is no evidence that debt financial flows reduce the global difference in the marginal product of capital. International trade also contributes to this convergence by equalizing international prices of investment and consumption goods.

The final essay estimates the effects of inflows of foreign physical capital on the output per capita growth. The essay uses an open economy extension of a neoclassical growth model to include the share of foreign physical capital in domestic investment. Inflows of machinery and capital equipment reinforce a positive growth in output per worker. Non-industrial (non-OECD) countries that rely on foreign high-quality capital grow faster. The findings suggest that policies should facilitate trade liberalization in developing countries and strengthen the domestic ability to absorb technological benefits from abroad. 


\section{Acknowledgments}

I would like to express my gratitude to my dissertation advisor and chair, Professor Shuichiro Nishioka, for taking me on as an advisee, for the guidance, time and constant support during my doctoral studies. This dissertation would not have been possible without his efforts in coauthoring the second essay.

I thank Ronald Balvers, Arabinda Basistha, Stratford Douglas and Alexander Kurov for being my dissertation committee and contributing with their in-depth discussion and valuable comments. Special thanks to Professor Alexei Egorov for numerous programming lessons and discussions leading into the development of my first chapter. I also would like to thank Camelia Minoiu, the economist at the International Monetary Fund, for reviewing my first chapter and providing excellent suggestions and comments.

I am grateful to the Economics Department of West Virginia University for the financial support, for providing me a place and resources to conduct my dissertation research, and for the opportunity to develop my teaching skills.

I thank my colleagues and friends, Julie Lohi, Eugene Bempong, Shadrack Mwilaria, Jorida Papakroni, Sheida Teimouri and Oleg Kucher for motivating and productive discussions, helpful comments and encouragement. I enjoyed working with them and I will truly miss them.

I would like to express gratitude to my dear friends, Natalia Pisareva, Elena Romanenko, Oxana Myachina, Alena Demidova, Pavel Zakovorotnyy and Fritz Campo for always being there for me and for sharing their knowledge and wisdom with me.

I would like to give my special thanks to my husband, Francisco Posada Sanchez, for his love and optimism. He has stood by my side from the earliest days of my Ph.D. studies and contributed to my personal and professional growth. His continuous kind 
support enable me to complete the dissertation.

I am deeply thankful to my parents, Nadezda and Georgy Bondarenko, for their love and encouragement. Their support is invaluable and my Ph.D. studies would not have been happen without it. 


\section{Contents}

Acknowledgments

List of Figures vii

List of Tables viii

1 Do Capital Controls Matter for Financial Integration? 1

1.1 Introduction . . . . . . . . . . . . . . . . 1

1.2 Data Sources. Description and Statistics . . . . . . . . . . . . 4

1.3 Empirical Analvsis $\ldots \ldots \ldots \ldots \ldots$

1.3 .1 Empirical Specification . . . . . . . . . . . . . . . 8

1.3.2 Estimation Results . . . . . . . . . . . . . . . . . . . . . 9

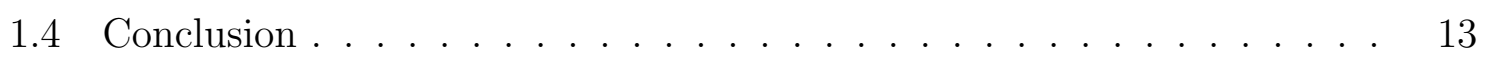

1.5 Tables and Figures $\ldots \ldots \ldots \ldots \ldots \ldots$

2 Determinants of Convergence in the Marginal Product of Capital 24

2.1 Introduction . . . . . . . . . . . . . . . . . . 24

2.2 Marginal Product of Capital $\ldots \ldots \ldots \ldots \ldots \ldots$

2.2 .1 Measures of MPK . . . . . . . . . . . . . . . . . . . 26

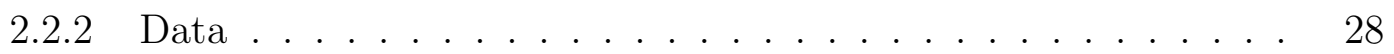

2.2 .3 Overview of MPK Convergence . . . . . . . . . . . . . . . . 29

2.3 Theorv for MPK Convergence . . . . . . . . . . . . . . . . . . . 30

2.3 .1 Domestic Factors . . . . . . . . . . . . . . . . . 30

2.3 .2 International Flows of Financial Assets . . . . . . . . . . . . 33

2.3 .3 International Trade . . . . . . . . . . . . . . . . . . 34

2.4 Empirical Evidence . . . . . . . . . . . . . . . . . . . . . . . . . . . 37

2.4 .1 Baseline Results . . . . . . . . . . . . . . . . . . . . . . 37

2.4 .2 Robustness Check . . . . . . . . . . . . . . . . . . . 41

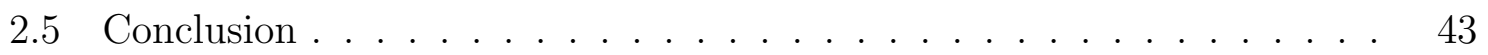

2.6 Tables and Figures $\ldots \ldots \ldots \ldots \ldots$ 
3 Capital Goods Inflows and Economic Growth 52

3.1 Introduction . . . . . . . . . . . . . . . . . . . . . 52

3.2 Neoclassical Growth Model . . . . . . . . . . . . . . . . . . . . 54

3.2.1 Growth Regression in a Closed Economy . . . . . . . . . . . 56

3.2.2 Augmenting the Solow Model with Inflows of Physical Capital . 57

3.3 Data . . . . . . . . . . . . . . . . . . . . . . . . . . . . . . . . . . . 58

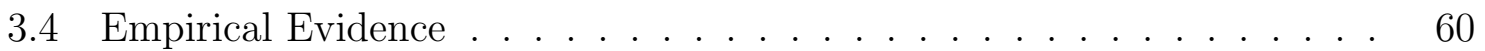

3.5 Conclusion . . . . . . . . . . . . . . . . . . . . . . . 64

3.6 Tables and Figures . . . . . . . . . . . . . . . . . . 65 


\section{List of Figures}

$1.1 \quad$ Evolution of Financial Integration: 1970-2004 . . . . . . . . . . . . 23

2.1 Sigma convergence of the marginal product of capital . . . . . . . . . 49

2.2 Beta convergence of the marginal product of capital . . . . . . . . . 49

2.3 Standard deviations of the saving rates across 52 countries $\ldots \ldots . \quad 50$

2.4 Standard deviations of the relative prices across 52 countries $\ldots \ldots .50$

$2.5 \mathrm{MPK}_{i+}$ and $\mathrm{KCT}_{i+} / \mathrm{K}_{i+}$ in $1970 \ldots \ldots \ldots \ldots \ldots \ldots \ldots$

$2.6 \quad \mathrm{MPK}_{i+}$ and $\mathrm{KCT}_{i+} / \mathrm{K}_{i+}$ in $2005 \ldots \ldots \ldots \ldots \ldots \ldots$

3.1 Real GDP per worker and Capital Goods Imports in 1975 . . . . . . 67

3.2 Real GDP per worker and Capital Goods Imports in 2005 . . . . . 67

3.3 Share of Capital Goods Export in Total Capital Exports. G6 countries 68 


\section{List of Tables}

1.1 Countrv List by Income Group . . . . . . . . . . . . . . . . . . . . . 15

1.2 Summarv Statistics . . . . . . . . . . . . . . . . . . . . 17

1.3 Correlation Matrix ...................... . . 18

1.4 Correlation between De-iure and De-facto Financial Openness . . . . . 19

1.5 De-Facto Conditional to De-iure and Domestic Factors. OLS (T=35) $\quad 20$

1.6 De-Facto Conditional to De-jure and Domestic Factors. LSDV (T=35) 21

1.7 De-Facto Conditional to De-jure and Domestic Factors, LSDV ( $\mathrm{T}=7)$. 22

2.1 Summarv Statistics . . . . . . . . . . . . . . . . . 45

2.2 MPK Convergence Conditional to Domestic and International Factors . 46

2.3 Financial Openness and Components of Financial Flows . . . . . . . . 47

2.4 Various measures of trade and trade openness . . . . . . . . . . . . . 48

3.1 Summarv Statistics . . . . . . . . . . . . . . . . 65

3.2 Economic Growth and Physical Capital Inflows: OLS and LSDV . . . . 65

3.3 Economic Growth and Physical Capital Inflows: GMM . . . . . . . 66 


\section{Chapter 1}

\section{Do Capital Controls Matter for Financial Integration?}

\subsection{Introduction}

The term 'financial integration' originated three decades ago, when several OECD countries and some developing nations started moving from financial repression towards financial liberalization by relaxing controls on national capital accounts. 11 Financial repression was characterized by fixed exchange rate regime, high reserve requirements, interest rate ceilings, and controls on capital flows in an effort to maintain financial stability. Such economies did not allow for efficient capital allocation, which higher capital flows flowing towards countries with higher interest rates. As a result, many countries experienced low growth, macroeconomic instability, and high costs to maintain regulations 2 The reasoning behind financial liberalization was to reduce costs related to maintaining fixed exchange rate and promote proper allocation of savings to productive investment, thus decreasing the effect of externalities caused by the regime. Many high-income OECD countries liberalized their financial systems during the 1980s, while

\footnotetext{
${ }^{1}$ See for instance Kose et al. (2009) on the evolution of international financial integration.

${ }^{2}$ See Johnston and Tamirisa (1998); Caprio et al. (2001) for more discussion on the characteristics and effects of financial repression.
} 
developing countries started the liberalization only in early 1990s and some emerging market economies just recently started liberalizing their capital accounts (IMF, 2007; OECD).

The empirical evidence on the effects of capital controls liberalization policy is mixed. A growing body of literature finds efficiency gains from capital account liberalization in developed countries in terms of economic growth, financial development, portfolio diversification, and reduction of the cost of capital. 3 On the contrary, there is an empirical support for emerging market economies that experience increased macroeconomic volatility and current account unbalances due to increased capital mobility 4 Therefore, some emerging countries reverse the trend toward the liberalization and impose controls on capital accounts. While previous studies focus primarily on the effects of financial liberalization and integration, the impact of capital controls on actual financial flows has not been the object of systematic econometric investigation so far. This paper addresses the empirical question of whether international financial flows are responsive to capital account restrictions or liberalization policies across countries with a different economic development level, and therefore, questions the practicality of capital controls as a policy tool.

I specify the importance of examining various components of total financial flows since factors influencing each type cross-border financial flows may be different. Many studies argue about the importance of studying disaggregated financial flows since they have different macroeconomic effects and benefits of financial globalization (e.g. Dell'Ariccia et al., 2007; Aizenman and Noy, 2009). While FDI flows exhibit stability, portfolio equity, bank loans and debt flows appear to be volatile bearing greater risk

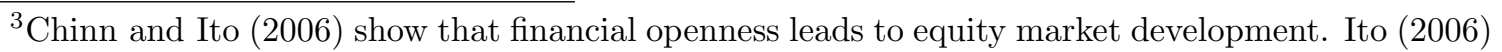
reconfirmed this finding for Asian region. Leahy (2001) documented the benefits of financial openness for OECD countries, noting that capital account liberalization leads to the development of financial markets.

${ }^{4}$ See for instance Dell'Ariccia et al. (2007). Stiglitz (2002) argues that capital market liberalization is not followed by growth, but lead to greater economic volatility and risks in developing countries. Edwards (2009) concludes that relaxing capital controls increase the probability of a sudden stop.
} 
for economic activity (Lane and Milesi-Ferretti, 2007). FDI is determined by long-term fundamental economic characteristics and thus more stable relative to other components of capital account, portfolio and short-term debt flows, called in the literature 'mobile capital' or 'hot money' (Rajan, 2002). FDI intensifies the productive ability of the host country and creates the necessary revenue to cover future capital outflows. That is why emerging countries that are more vulnerable to sudden stops and currency crisis tend to have higher share of short-term external debt and smaller share of FDI in aggregated capital flows (Frankel and Rose, 1996). The disaggregation of financial flows thus provides more detailed information about possible channels of financial integration and potential risks associated with them.

The paper involves three disaggregated measures of financial openness: FDI, portfolio equity and debt flows. I examine whether an increase in FDI and portfolio equity flows is more likely to occur in liberalized financial systems, while debt flows are more likely to prevail in financially closed economies, after controlling for domestic factors that play a significant role in the financial liberalization process. The literature suggests to take into account persistent country characteristics like openness to trade, level of economic development, and balance of payments environment when policy decisions are made regarding financial openness strategies (Aizenman and Noy (2009), Chinn and Ito (2006), Dell'Ariccia et al. (2007), Johnston and Tamirisa (1998), Leblang (1997)). I consider four country groups according to their economic development level: high-income (OECD and non-OECD), middle income, low income countries and a full sample. The analysis allows to differentiate the responsiveness of financial flows to capital controls policies among country groups with similar financial market characteristics. I employ trade openness, real GDP per capita and total reserves in months of imports as fundamental factors contributing to international capital mobility. I develop a data set of 129 countries for the period from 1970 to 2004.

The empirical evidence suggests that impact of capital account restrictions differs across country groups and types of financial flows. While capital controls are more 
likely to be effective towards all types of international capital flows in high-income countries, they have an insignificant impact for FDI and equity flows in low-income countries. The results are consistent with the prominent studies that investigate the effectiveness of capital controls in selected countries 5 Low-income countries can be financially integrated in the presence of capital controls or, vice-versa, countries with liberalized capital accounts are not necessarily financially integrated. The important issue to understand is why some economies attain higher level of financial integration while maintaining high levels of controls on capital account transactions. This paper suggest that the effectiveness of capital controls can explain the discrepancy between the policy and actual financial flows. Understanding the accompanying determinants of financial openness is critical in creating an appropriate direction for the capital controls policies so that their effectiveness and benefits can be maximized.

\subsection{Data Sources, Description and Statistics}

The panel data is based on annual observation for 129 countries over the period 19702004, splitting the sample in three income groups. Countries are listed in the Table 1.1. Income groups are classified according to World Bank's World Development Indicators (WDI) country classifications. Actual cross-border financial flows are defined in the literature as de-facto financial openness. I obtain the data on the de-facto financial openness from Lane and Milesi-Ferretti (2007), who constructed the dataset on gross liabilities and assets positions for 145 nations over the 1970-2004 period. The gross defacto financial openness is the sum of assets and liabilities of portfolio equity, FDI, debt, financial derivatives and total reserves minus gold, which represent both, international

\footnotetext{
5 Vlades-Prieto and Soto (1998) Cardenas and Barrera (1997), and Bush and Hanschel (2000) conduct country studies for Chile and Colombia, and Slovenia, correspondingly, and find that find controls are ineffective in achieving their objectives. Aizenman and Noy (2009) investigate the effect of capital account restrictions on aggregate financial flows across developing countries and argue that capital controls have only a weak impact on the level of cross-border financial flows.
} 
transactions and holdings between a country's residents and nonresidents 6 I use the sum of assets and liabilities as a percentage to GDP to capture total and individual cross-border financial flows. Gross flows, in general, argued to provide a less volatile and more sensible picture of financial openness, and do not tend to have measurement error when used as a ratio to GDP (Kose et al., 2009).

To account for capital controls, I use the index, KAOPEN, developed by Chinn and Ito (2008) that measures the openness or restrictions to capital account transactions.7 The index is constructed based on the IMF's Annual Report on Exchange Arrangements and Exchange Restrictions (AREAER) and capture the intensity of administrative restrictions on cross-border capital flows. KAOPEN represents the first principal component of four IMF binary variables: existence of multiple exchange rates, restrictions on current account transaction, restriction on capital account transactions, and requirements of the surrender of export proceeds, therefore referring to the existence of different types of restrictions. The index ranges from -2.5 to 2.5 , from full control to complete liberalization correspondingly. Measure of legal restrictions on cross-border capital flows are referred in the literature as de-jure financial openness.

While the de-jure financial openness reflects a country's decision to open its economy to international financial capital flows, the de-facto measure shows the degree of the stock market integration and capitalization as a result of interaction between the implementation of capital account restrictions and market forces. Figure 1.1 depicts

\footnotetext{
${ }^{6}$ See Lane and Milesi-Ferretti (2007). Thus, portfolio inflows refer to foreign investors' purchases of domestically issued equity in a company. They include the sum of country funds, depository receipts, and direct purchases of shares by foreign investors. Portfolio equity holdings measure ownership of shares of companies and mutual funds that are below 10\% threshold level. FDI refers to direct investment in a domestic company, including controlling stakes in addition to $10 \%$ Greenfield investment (minority stakes). Debt category includes debt securities, bank loans and deposits, and other debt instruments. Thus, debt inflow includes foreign investors' purchases of debt issued by corporate or the government, and also foreign borrowing undertaken by domestic banks. Financial derivatives refer to the market value of the outstanding derivatives contracts.

[Chinn and Ito (2008) by constructing the capital account openness index, provided the comparative illustration of financial openness of most countries around the world. They note that the majority of the developed industrial countries have high level of financial liberalization; developing and emerging markets have less capital account liberalization implying that countries experiencing significant restrictions on financial flows.
} 
the evolution of financial openness and offers insights into how capital account liberalization and financial integration differ across countries. The general trend across countries suggests increasing financial integration over the period 1970 to 2004, however figures generally do not point to causality from de-jure restrictions to de-facto financial flows 8 High-income countries have been experiencing significant growth in both de-jure and de-facto openness measures, becoming more financially liberalized and integrated over time. Middle-income countries have been removing their restrictions on capital account transaction, having de-jure measure raised substantially, however have not experienced much growth in actual financial flows. In low-income countries, de-jure financial openness has remained almost the same, indicating the presence of strong capital controls policies, while actual flows experienced moderate growth, by a level greater than in middle-income countries in absolute terms 9 This evidence is consistent with Kose et al. (2009) who note that Latin American countries have experienced an increasing average volume of actual cross-border financial flows over the past two decades despite having a high level of capital account restrictions. In contrast, some African countries have few restrictions on capital account transactions, but do not attract significant cross-border capital flows.

I consider three fundamental factors that determine a country's level of financial openness, its ability to reduce capital controls and to benefit from financial integration with minimal risks for its economy: trade openness, economic development level and international reserves. The data is obtained from the World Bank Development Indicators Database. The trade openness index is constructed as the sum of imports and exports (percentage of GDP); gross international reserves in months of imports are given as a ratio of gross international reserves to the current U.S. dollar value of imports of goods and services, multiplied by 12. Gross international reserves consists

\footnotetext{
${ }^{8}$ Trend analysis determine that the global movements of financial flows exhibit increasing trend over time, with the highest trend in high-income countries.

${ }^{9}$ Kose et al. (2009) describe similar pattern of financial openness, using IMF binary 0-1 capital account restrictiveness classification, where 1 represent countries that have open capital accounts.
} 
of monetary gold, special drawing rights (SDRs), the reserve position of member with the IMF, and holdings of foreign exchange under the control of monetary authorities. Per capita GDP in constant 2000 US dollars defines the economic development level.

The data set is an unbalanced panel consisting of about 4515 observations. Table 1.2 reports summary statistics for the variables of interest in 1970 and 2004. International financial and trade flows are included as shares of GDP. In 1970, the lowest and the highest values of gross de-facto financial flows are in Iran (0.03) and Malta (4.90), correspondingly. In 2005, Bahrain (19.16) obtains the highest gross de-facto financial flows, while Belarus (0.39) maintain the lowest financial flows across all countries. A number of low-income countries report zero financial flows in 1970, therefore the minimum level of disaggregated financial flows (FDI, portfolio equity and debt) in 1970 are assumed to be zero. The highest levels of FDI, portfolio equity and debt are reported in Panama (1.15), Netherlands (0.27) and United Arab Emirates (4.81), correspondingly. The United States, Japan and a number of high-income countries maintain the highest degree of financial liberation (2.53) in 1970 and 2005. The highest restrictions on capital accounts are found in Colombia (-1.81) in 1970 and in Zimbabwe (-1.81) and other low-income countries. Overall, the summary statistics suggest that high-income countries are financially liberalized and integrated. Low-income and middle-income countries maintain conservative financial openness.

Table 1.3 reports correlations among variables of interest. All coefficients have the expected signs. Correlations between capital controls and international financial flows are positive and imply that the less domestic financial markets are restricted, the more they are integrated into international financial market. Positive correlations between trade openness and de-facto financial openness suggest that increase in international commodity markets promote higher cross-border financial flows. Total reserves in months of imports tend to be higher when domestic capital controls are lower, that is when de-jure financial openness index is higher, and when GDP per capita is higher. Typically, the GDP level is higher in financially open economies: GDP per capita cor- 
relates positively with de-jure and de-facto financial openness. FDI and equity flows are influenced by de-jure restrictions more compared to debt flows. Table 1.4 reports the correlations between de-jure and de-facto financial openness in different country groups. The relationship between legal restrictions on capital accounts and actual financial flows are strong, positive and significant in high-income and middle-income countries across the majority of financial flows. However, the relationship is weaker and insignificant in low-income countries. This evidence signals that financial flows may be not responsive to capital controls in low-income countries.

\subsection{Empirical Analysis}

\subsubsection{Empirical Specification}

I aim to empirically examine whether legal restrictions on the capital account have an effect on cross-border financial flows. The following log-linear specification is considered:

$$
\begin{aligned}
\ln \left(\text { DeFacto }_{i, t}^{X}\right)= & \beta_{1} \ln \left(\text { DeJure }_{i, t}\right)+\beta_{2} \ln (\text { TRADE } \\
& \left.+\beta_{4, t}\right)+\beta_{3} \ln \left(\text { PCGM }_{i, t}\right)+\xi_{t}+\eta_{i}+\epsilon_{i t}
\end{aligned}
$$

where the dependent variable, DeFacto, is a measure of gross financial flows for country $i$ at the time $t$ of the flow type $X$ (total flows, FDI, portfolio equity, and debt as shares of GDP); DeJure is a capital controls index; TRADE is trade openness (a share of GDP), TRMI is total reserves in months of imports; PCGGP is per capital GDP. $\xi_{t}$ represents the time fixed effect, $\eta_{i}$ accounts for the country-fixed effect or unobserved factors, such as institutions and resources. $\epsilon_{i t}$ is a time-varying error term.

By estimating equation 1.1, the paper aims to answer which subcomponent of financial flows is responsive to a greater degree of capital account restrictions. I expect 
a positive relationship between de-facto financial flows and de-jure financial openness. Lower restrictions on capital account should lead to a higher level of cross-border financial flows and visa-versa.

\subsubsection{Estimation Results}

I start estimation of the equation (1.1) by ordinary least squares (OLS) estimator including time dummy variables. The results are reported in Table (1.5). The table contains the estimates for different income group countries. There is positive and statistically significant at the $1 \%$ condifidence level relationship between different types of de-facto financial flows and de-jure financial openness in full sample. The relationship is strong in high-income countries. A 1\% increase in de-jure financial openness is associated with a $0.35 \%$ increase in aggregated de-facto financial openness, a $0.41 \%$ increase in FDI flows, a $0.55 \%$ increase in portfolio equity flows, and a $0.33 \%$ increase in debt flows. Positive and statistically significant coefficient on capital account restrictions for debt flows may imply the willingness and ability of advanced economies to serve as a lender to developing countries. In middle-income countries, the relationship between capital controls and financial flows are weaker and less significant. A 1\% increase in de-jure financial openness leads to a $0.06 \%$ increase in FDI flows and a $0.09 \%$ increase in debt flows. The coefficient on equity flows is negative and insignificant.

In low-income countries, the coefficients on the de-jure financial openness is negative and statistically significant for aggregate flows, portfolio equity and debt flows: a $1 \%$ increase in the capital controls index is associated with a $0.09 \%, 0.66 \%$ and a $0.11 \%$ decrease in aggregate flows, equity and debt flows, correspondingly. It is worth noting that financial liberalization may have different effects on various parts of the capital accounts in different countries. A negative coefficient of de-jure financial openness in equity and debt equations can be explained by countries' preferences to become less dependent on volatile flows including foreign loans as they liberalize their financial mar- 
kets. As countries become more integrated into international financial markets, they reduce the level of foreign loans, or visa-versa, as countries impose more restrictions on capital account transactions, they rely more on foreign loans. FDI flows appear not to be responsive to capital controls: the coefficient on de-jure controls is positive, but insignificant. Overall, the OLS results confirm the hypothesis and suggest that international financial flows are responsive to national capital account policies in developed countries. Financial flows appear to be less responsive to the capital controls in middle- and low-income countries. However, OLS estimates can be biased due to the correlation between the country specific fixed effect and explanatory variables.

Next, I estimate the equation (1.1) by least squared dummy variables (LSDV) estimator including time- and country-specific dummy variables. The estimator is robust given the presence of heteroscedastic and contemporaneously cross-country correlated disturbances. It reduces the measurement error and omitted variables bias. Table (1.6) reports the results. In high-income countries, FDI, equity and debt flows are responsive to capital account policies. A $1 \%$ increase in de-jure financial openness is associated with a $0.18 \%$ increase in aggregated de-facto financial openness, a $0.16 \%$ increase in FDI flows, a $0.22 \%$ increase in equity, and a $0.18 \%$ increase in debt flows. In comparison to corresponding OLS results, the responce of financial flows to regulations on capital and current account are lower. In middle-income countries, the coefficients on de-jure capital controls are positive and statistically significant for FDI and equity flows. A $1 \%$ increase in capital account index implies a $0.05 \%$ increase in FDI flows and a $0.16 \%$ increase in equity flows. However, the coefficients on aggregate financial flows and debt flows are negative. Similar to OLS results, the magnitutes of de-jure coefficients are lower in middle-income countries compared to high-income countries. In low-income countries, while the coefficient on the de-jure financial openness is insignificant for FDI and equity flows, it is negative and statistically significant for debt flows. A $1 \%$ increase in the capital account index leads to a $0.09 \%$ decrease in debt flows. A negative and significant coefficient on de-jure financial openness in the aggregate de-facto openness 
equation may be influenced by the debt and equity flows. It is not a surprising result given that the share of debt flows in gross assets and liabilities remains very high for low-income countries. The coefficients on de-jure controls exhibit similar values to coefficients obtained by OLS in all equations exept for equity equiation.

Further, I employ an alternative data set by transforming the original panel data set into seven non-overlapping five-year averages 10 The transformation filters out business cycle fluctuations, and allows to examine long-run growth effects. I estimate the equation (1.1) by OLS and LSDV. The results are statistically significant and qualitatively similar to the results obtained using the non-transformed data. The results derived by LSDV estimator are reported in Table (1.7). Coefficients on the de-jure financial openness in FDI and equity equations are positive and statistically significant in high-income, middle-income countries and in full sample. However, de-jure coefficients in de-facto and debt equations are negative in middle-income, low-income countries and in full sample. FDI and equity flows appear not to be responsive to capital controls in low-income countries, despite high levels of capital controls. The empirical evidence suggests that capital controls matter for financial integration in high- and middle-income countries.

The OLS and LSDV estimates are robust to alternative econometric specification and sample selection. I perform several robustness checks. First, I test the sensitivity of the de-jure coefficients across all countries and types of financial flows. The coefficients are statistically significant and consistent. Next, I test the hypothesis of joint importance of explanatory variables for the financial integration. I examine interaction terms between de-jure financial openness and international trade, per capita GDP and total reserves in months of imports. The results show that international trade and economic development level reinforce the relationship between de-jure and de-facto financial openness across all countries. Total reserves in months of imports do

\footnotetext{
${ }^{10} \mathrm{It}$ is standard in the literature to transform panel data, especially the one that contains volatile financial flows, into five-year averages. See for instance Aghion et al. (2009).
} 
not have significant influence jointly with de-jure financial openness on financial integration in high and middle-income countries, however have a significant effect in lowincome countries. Further, I consider dynamic model specification by including lagged dependent variable, thus the dependent variable is a function of its own past values. I employ the two-step generalized method of moments (GMM) estimator proposed by Arellano and Bond (1991) for five-year non-overlapping averages data.11 GMM estimators are designed to cope with problems of serial correlation and heteroscedasticity. The GMM estimator eliminates any endogeneity that may be due to the correlation between country specific effects and regressors. Moreover, GMM estimator differences model variables and that removes time-invariant country specific variable. All righthand side variables are treated as endogenous variables and lagged level of endogenous variables are used as instruments. The specification tests reveal that the errors are not serially correlated and the instruments are exogenous. The results obtained by GMM estimator are consistent with the main results and robust to minor model modifications. While capital controls matter for international financial integration in high-and middle-income countries, they appear less relevant in low-income economies.

Across all models, the results show a positive and significant effect of international trade on financial integration across all countries. Trade openness provides greater potential for financial liberalization and promotes productive FDI flows that should help an economy to serve its debt (Kose et al., 2009). The results are consistent with the findings of Blonigen (2001), Aizenman (2008) and Aizenman and Noy (2006) who argue that international trade and foreign direct investment are positively associated. The level of economic development and total reserves in months of imports have a significant and economically meaningful effect on cross-border financial flows. Countries with higher GDP per capita tend to be more financially integrated than those with lower levels of GDP per capita and thus, higher levels of debt flows. The effect of development

\footnotetext{
${ }^{11}$ The Arellano-Bond estimator is suitable for a relatively short time dimension $\mathrm{T}$, and a larger country dimension, N. In all subsamples, the conditions $\mathrm{N}>\mathrm{T}$ is satisfied.
} 
level of a country on international financial integration is generally stronger in advanced economies and weaker in developing countries, with the effect being positive for equity investment. In low-income countries, however, per capital GDP is negatively related to debt flows, suggesting that countries will reduce the level of foreign debt obligations when economic development conditions improves. Furthermore, reserves in months of imports have negative and significant coefficients for most financial flows across all groups of countries implying that the reduction in foreign reserves is associated with the growth in cross-border financial flows.

\subsection{Conclusion}

World financial markets have grown rapidly over the past three decades, engaging more countries-participants with liberalized capital accounts in international financial operations. The results are consistent with the notion that advanced economies become more liberalized and integrated into global financial markets, while emerging market economies continue to maintain conservative capital controls. The analysis presented in this paper suggests that there are some common factors that can explain the pattern of financial integration, but individual country circumstances may vary. Factors like openness to trade, international reserves, and economic development level strengthen a country's ability to manage international capital flows and benefit from financial integration. However, capital account restrictions have mixed effects on financial integration across different groups of countries. The degree of legal enforcement of capital controls matter for the level of financial integration in advanced countries, however controls have an insignificant effect on de-facto financial openness in developing countries. Large capital flows in the presence of restrictions on capital account transactions may imply that agents in some countries attempt to circumvent capital controls, since capital restrictions may deny individuals the opportunity to carry out profitable transactions. 
Many studies stress the necessity to address policy implications when analyzing the importance of capital controls and effects of financial liberalization. While the main objective of the paper is to offer empirical evidence, some general principles emerge from the analysis about how countries can increase benefits from financial openness. A country can benefit by accessing the effectiveness of controls on international financial flows, especially volatile and risky portfolio equity and debt flows. Effective capital account controls should restrict large outflows of capital, prevent nominal currency depreciation, lower nonproductive, short-term capital inflows and generally reduce the probability of a financial crisis. On the contrary, financial flows that are not responsive to capital account restrictions can result in large fluctuations in international reserves and interest rates. Thus, by reconsidering controls on capital transactions, that is promoting FDI and restricting equity or debt flows, a country can be more prepared to manage large international financial flows and avoid negative consequences of financial liberalization. Capital controls can be an effective policy tool in countries with liberalized international trade, a high economic development level and adequate reserves. 


\subsection{Tables and Figures}

Table 1.1: Country List by Income Group

\begin{tabular}{|c|c|c|c|c|c|}
\hline Country & WB Code & IFS Code & Country & WB Code & IFS Code \\
\hline \multicolumn{6}{|c|}{ High Income OECD Countries } \\
\hline Australia & AUS & 193 & Japan & JPN & 158 \\
\hline Austria & AUT & 122 & Korea, Rep. & KOR & 542 \\
\hline Belgium & BEL & 124 & Netherlands & NLD & 138 \\
\hline Canada & CAN & 156 & New Zealand & NZL & 196 \\
\hline Denmark & DNK & 128 & Norway & NOR & 142 \\
\hline Finland & FIN & 172 & Portugal & PRT & 182 \\
\hline France & FRA & 132 & Spain & ESP & 184 \\
\hline Germany & DEU & 134 & Sweden & SWE & 144 \\
\hline Greece & GRC & 174 & Switzerland & $\mathrm{CHE}$ & 146 \\
\hline Iceland & ISL & 176 & United Kingdom & GBR & 112 \\
\hline Ireland & IRL & 178 & United States & USA & 111 \\
\hline Italy & ITA & 136 & & & \\
\hline \multicolumn{6}{|c|}{ High Income non-OECD Countries } \\
\hline Bahrain & BHR & 419 & Qatar & QAT & 453 \\
\hline Cyprus & CYP & 423 & Saudi Arabia & SAU & 456 \\
\hline Hong Kong, China & HKG & 532 & Singapore & SGP & 576 \\
\hline Israel & ISR & 436 & Slovenia & SVN & 961 \\
\hline Kuwait & KWT & 443 & United Arab Emirates & ARE & 466 \\
\hline Malta & MLT & 181 & & & \\
\hline \multicolumn{6}{|c|}{ Upper Middle Income Countries } \\
\hline Argentina & ARG & 213 & Malaysia & MYS & 548 \\
\hline Botswana & BWA & 616 & Mauritius & MUS & 684 \\
\hline Chile & CHL & 228 & Mexico & MEX & 273 \\
\hline Costa Rica & CRI & 238 & Oman & OMN & 449 \\
\hline Croatia & HRV & 960 & Panama & PAN & 283 \\
\hline Czech Republic & CZE & 935 & Poland & POL & 964 \\
\hline Estonia & EST & 939 & Slovak Republic & SVK & 936 \\
\hline Gabon & GAB & 646 & South Africa & $\mathrm{ZAF}$ & 199 \\
\hline Hungary & HUN & 944 & Trinidad and Tobago & TTO & 369 \\
\hline Latvia & LVA & 941 & Turkey & TUR & 186 \\
\hline Lebanon & LBN & 446 & Uruguay & URY & 298 \\
\hline Libya & LBY & 672 & Venezuela, RB & VEN & 299 \\
\hline Lithuania & LTU & 946 & & & \\
\hline \multicolumn{6}{|c|}{ Lower Middle Income Countries } \\
\hline Albania & ALB & 914 & Honduras & HND & 268 \\
\hline
\end{tabular}


Table 1.1: (continued)

\begin{tabular}{|c|c|c|c|c|c|}
\hline Country & WB Code & IFS Code & Country & WB Code & IFS Code \\
\hline Algeria & DZA & 612 & Indonesia & IDN & 536 \\
\hline Angola & AGO & 614 & Iran, Islamic Rep. & IRN & 429 \\
\hline Armenia & $\mathrm{ARM}$ & 911 & Jamaica & JAM & 343 \\
\hline Azerbaijan & AZE & 912 & Jordan & JOR & 439 \\
\hline Belarus & BLR & 913 & Morocco & MAR & 686 \\
\hline Bolivia & $\mathrm{BOL}$ & 218 & Namibia & NAM & 728 \\
\hline Brazil & BRA & 223 & Paraguay & PRY & 288 \\
\hline Bulgaria & BGR & 918 & Peru & PER & 293 \\
\hline China & $\mathrm{CHN}$ & 924 & Philippines & PHL & 566 \\
\hline Colombia & $\mathrm{COL}$ & 233 & Romania & ROM & 968 \\
\hline Dominican Republic & DOM & 243 & Sri Lanka & LKA & 524 \\
\hline Ecuador & $\mathrm{ECU}$ & 248 & Swaziland & SWZ & 734 \\
\hline Egypt, Arab Rep. & EGY & 469 & Syrian Arab Republic & SYR & 463 \\
\hline El Salvador & SLV & 253 & Thailand & THA & 578 \\
\hline Fiji & FJI & 819 & Tunisia & TUN & 744 \\
\hline Guatemala & GTM & 258 & Ukraine & UKR & 926 \\
\hline \multicolumn{6}{|c|}{ Low Income Countries } \\
\hline Bangladesh & BGD & 513 & Mozambique & $\mathrm{MOZ}$ & 688 \\
\hline Benin & BEN & 638 & Myanmar & MMR & 518 \\
\hline Burkina Faso & BFA & 748 & Nepal & NPL & 558 \\
\hline Cote d'Ivoire & CIV & 662 & Nicaragua & NIC & 278 \\
\hline Cameroon & CMR & 622 & Niger & NER & 692 \\
\hline Chad & TCD & 628 & Nigeria & NGA & 694 \\
\hline Congo, Dem. Rep. & ZAR & 636 & Pakistan & PAK & 564 \\
\hline Congo, Rep. & COG & 634 & Papua New Guinea & PNG & 853 \\
\hline Ethiopia & ETH & 644 & Rwanda & RWA & 714 \\
\hline Ghana & GHA & 652 & Senegal & SEN & 722 \\
\hline Guinea & GIN & 656 & Sudan & SDN & 732 \\
\hline Haiti & HTI & 263 & Tanzania & TZA & 738 \\
\hline India & IND & 534 & Togo & TGO & 742 \\
\hline Kenya & KEN & 664 & Uganda & UGA & 746 \\
\hline Madagascar & MDG & 674 & Vietnam & VNM & 582 \\
\hline Malawi & MWI & 676 & Yemen, Rep. & YEM & 474 \\
\hline Mali & MLI & 678 & Zambia & $\mathrm{ZMB}$ & 754 \\
\hline Moldova & MDA & 921 & Zimbabwe & ZWE & 698 \\
\hline
\end{tabular}


Table 1.2: Summary Statistics

\begin{tabular}{lllll}
\hline \hline & Mean & Std. Dev. & Min & Max \\
\hline \hline Year: 1970 & & & & \\
De-Facto Openness (\%, GDP) & 0.63 & 0.66 & 0.03 (Iran) & 4.90 (Malta) \\
FDI (\%, GDP) & 0.13 & 0.18 & - & 1.15 (Panama) \\
Portfolio Equity (\%, GDP) & 0.01 & 0.04 & - & 0.27 (Netherlands) \\
Debt (\%, GDP) & 0.46 & 0.69 & - & 4.81 (United Arab Emirates) \\
De-Jure Openness & -0.39 & 1.28 & -1.81 (Colombia) & 2.53 (United States) \\
Trade (\%, GDP) & 0.51 & 0.30 & 0.05 (China) & 1.79 (Hong Kong) \\
Real GDP per capita (\$US, 2000=100) & 4460.34 & 6446.50 & 121.60 (Malawi) & 39795.04 (Kuwait) \\
Total Reserves in Months of Imports & 2.62 & 1.22 & 0.99 (Dominican Republic) & 4.91 (Austria) \\
& & & & \\
Year:2004 & & & & \\
De-Facto Openness (\%, GDP) & 2.49 & 3.00 & 0.39 (Belarus) & 19.16 (Bahrain) \\
FDI (\%, GDP) & 0.53 & 0.63 & 0.02 (Iran) & 5.20 (Hong Kong) \\
Portfolio Equity (\%, GDP) & 0.25 & 0.59 & - & 17.10 (Ireland) \\
Debt (\%, GDP) & 1.50 & 2.12 & 0.21 (India) & 2.53 (Japan) \\
De-Jure Openness & 0.77 & 1.62 & -1.81 (Zimbabwe) & 4.33 (Singapore) \\
Trade (\%, GDP) & 0.89 & 0.55 & 0.25 (United States) & 39352.70 (Norway) \\
Real GDP per capita (\$US, 2000=100) & 7487.89 & 10150.56 & 88.02 (Congo) & 24.87 (Libya) \\
Total Reserves in Months of Imports & 4.43 & 3.53 & 0.18 (Ireland) & \\
\hline \hline
\end{tabular}


Table 1.3: Correlation Matrix

\begin{tabular}{|c|c|c|c|c|c|c|c|c|}
\hline & De-Facto & FDI & Equity & Debt & De-Jure & Trade & PCGDP & TRMI \\
\hline De-Facto Openness (\%, GDP) & 1 & & & & & & & \\
\hline FDI $(\%$, GDP $)$ & 0.479 & 1 & & & & & & \\
\hline Portfolio Equity (\%, GDP) & 0.439 & 0.544 & 1 & & & & & \\
\hline Debt $(\%$, GDP $)$ & 0.977 & 0.304 & 0.283 & 1 & & & & \\
\hline De-Jure Openness & 0.344 & 0.336 & 0.333 & 0.287 & 1 & & & \\
\hline Trade $(\%$, GDP) & 0.503 & 0.577 & 0.297 & 0.415 & 0.268 & 1 & & \\
\hline Real GDP per capita ( $\$$ US, $2000=100)$ & 0.251 & 0.307 & 0.483 & 0.175 & 0.588 & 0.145 & 1 & \\
\hline Total Reserves in Months of Imports & -0.026 & -0.040 & 0.002 & -0.061 & 0.019 & -0.019 & 0.032 & 1 \\
\hline
\end{tabular}


Table 1.4: Correlation between De-jure and De-facto Financial Openness

\begin{tabular}{lcccc}
\hline \hline & Full sample & Hign Income & Middle Income & Low Income \\
\hline De-Facto Openness (\%, GDP) & 0.3443 & 0.3149 & 0.3179 & 0.013 \\
FDI (\%, GDP) & 0.3362 & 0.3300 & 0.2297 & 0.1095 \\
Portfolio Equity (\%, GDP) & 0.3330 & 0.3339 & 0.0043 & -0.0582 \\
Debt (\%, GDP) & 0.2875 & 0.2658 & 0.2717 & -0.0229 \\
\hline
\end{tabular}


Table 1.5: De-Facto Conditional to De-jure and Domestic Factors, OLS ( $\mathrm{T}=35$ )

\begin{tabular}{|c|c|c|c|c|c|c|c|c|c|}
\hline & & \multicolumn{4}{|c|}{ All Countries } & \multicolumn{4}{|c|}{ High-Income Countries } \\
\hline & & $\ln \left(\right.$ DeFacto $\left._{i, t}\right)$ & $\ln \left(F D I_{i, t}\right)$ & $\ln \left(\right.$ Equity $\left._{i, t}\right)$ & $\ln \left(\right.$ Debt $\left._{i, t}\right)$ & $\ln \left(\right.$ DeFacto $\left._{i, t}\right)$ & $\ln \left(F D I_{i, t}\right)$ & $\ln \left(\right.$ Equity $\left._{i, t}\right)$ & $\ln \left(\right.$ Debt $\left._{i, t}\right)$ \\
\hline $\ln \left(\right.$ DeJure $\left._{i, t}\right)$ & $\begin{array}{c}\beta_{1} \\
\text { (std error) }\end{array}$ & $\begin{array}{c}0.064^{* * *} \\
(0.013)\end{array}$ & $\begin{array}{c}0.104^{* * *} \\
(0.026)\end{array}$ & $\begin{array}{c}0.072 \\
(0.058)\end{array}$ & $\begin{array}{c}0.062^{* * *} \\
(0.014)\end{array}$ & $\begin{array}{c}0.357^{* * *} \\
(0.038)\end{array}$ & $\begin{array}{c}0.415^{* * *} \\
(0.046)\end{array}$ & $\begin{array}{c}0.558^{* * *} \\
(0.094)\end{array}$ & $\begin{array}{c}0.333^{* * *} \\
(0.043)\end{array}$ \\
\hline $\ln \left(\right.$ Trade $\left._{i, t}\right)$ & $\begin{array}{c}\beta_{2} \\
\text { (std error) }\end{array}$ & $\begin{array}{c}0.643^{* * *} \\
(0.022)\end{array}$ & $\begin{array}{c}0.782^{* * *} \\
(0.028)\end{array}$ & $\begin{array}{c}0.553^{* * *} \\
(0.067)\end{array}$ & $\begin{array}{c}0.553^{* * *} \\
(0.026)\end{array}$ & $\begin{array}{c}1.048^{* * *} \\
(0.038)\end{array}$ & $\begin{array}{c}0.635^{* * *} \\
(0.048)\end{array}$ & $\begin{array}{c}0.605^{* * *} \\
(0.066)\end{array}$ & $\begin{array}{c}1.101^{* * *} \\
(0.047)\end{array}$ \\
\hline $\ln \left(P C G D P_{i, t}\right)$ & $\begin{array}{c}\beta_{3} \\
\text { (std error) }\end{array}$ & $\begin{array}{c}0.088^{* * *} \\
(0.006)\end{array}$ & $\begin{array}{c}0.162^{* * *} \\
(0.012)\end{array}$ & $\begin{array}{c}0.888^{* * *} \\
(0.033)\end{array}$ & $\begin{array}{c}0.068^{* * *} \\
(0.007)\end{array}$ & $\begin{array}{c}0.027 \\
(0.050)\end{array}$ & $\begin{array}{c}0.108 \\
(0.083)\end{array}$ & $\begin{array}{c}1.007^{* * *} \\
(0.117)\end{array}$ & $\begin{array}{c}0.028 \\
(0.054)\end{array}$ \\
\hline $\ln \left(T R M I_{i, t}\right)$ & $\begin{array}{c}\beta_{4} \\
\text { (std error) }\end{array}$ & $\begin{array}{c}-0.054^{* * *} \\
(0.012)\end{array}$ & $\begin{array}{c}-0.079 * * * \\
(0.017)\end{array}$ & $\begin{array}{l}-0.063 \\
(0.044)\end{array}$ & $\begin{array}{c}-0.140^{* * *} \\
(0.014)\end{array}$ & $\begin{array}{l}-0.046 \\
(0.028)\end{array}$ & $\begin{array}{c}-0.226^{* * *} \\
(0.047)\end{array}$ & $\begin{array}{c}-0.242^{* * *} \\
(0.078)\end{array}$ & $\begin{array}{c}-0.083^{* * *} \\
(0.032)\end{array}$ \\
\hline Constant & & $\begin{array}{c}1.211^{* * *} \\
(0.156)\end{array}$ & $\begin{array}{c}-1.237^{* * *} \\
(0.275)\end{array}$ & $\begin{array}{c}-8.564^{* * *} \\
(0.634)\end{array}$ & $\begin{array}{c}1.596^{* * *} \\
(0.192)\end{array}$ & $\begin{array}{c}0.000 \\
(0.567)\end{array}$ & $\begin{array}{c}0.175 \\
(1.047)\end{array}$ & $\begin{array}{c}-9.516^{* * *} \\
(1.297)\end{array}$ & $\begin{array}{l}-0.523 \\
(0.613)\end{array}$ \\
\hline \multirow[t]{3}{*}{ Adj. R-squares } & & $\begin{array}{l}2,967 \\
0.532\end{array}$ & $\begin{array}{l}2,903 \\
0.467\end{array}$ & $\begin{array}{l}1,893 \\
0.439\end{array}$ & $\begin{array}{l}2,967 \\
0.389\end{array}$ & $\begin{array}{c}894 \\
0.692\end{array}$ & $\begin{array}{c}864 \\
0.566\end{array}$ & $\begin{array}{c}778 \\
0.552\end{array}$ & $\begin{array}{c}894 \\
0.618\end{array}$ \\
\hline & & \multicolumn{4}{|c|}{ Middle-Income Countries } & \multicolumn{4}{|c|}{ Low-Income Countries } \\
\hline & & $\ln \left(\right.$ DeFacto $\left._{i, t}\right)$ & $\ln \left(F D I_{i, t}\right)$ & $\ln \left(\right.$ Equity $\left._{i, t}\right)$ & $\ln \left(\right.$ Debt $\left._{i, t}\right)$ & $\ln \left(\right.$ DeFacto $\left._{i, t}\right)$ & $\ln \left(F D I_{i, t}\right)$ & $\ln \left(\right.$ Equity $\left._{i, t}\right)$ & $\ln \left(\right.$ Debt $\left._{i, t}\right)$ \\
\hline $\ln \left(\right.$ DeJure $\left._{i, t}\right)$ & $\begin{array}{c}\beta_{1} \\
\text { (std error) }\end{array}$ & $\begin{array}{c}0.060^{* * *} \\
(0.016)\end{array}$ & $\begin{array}{l}0.063^{*} \\
(0.033)\end{array}$ & $\begin{array}{l}-0.105 \\
(0.074)\end{array}$ & $\begin{array}{c}0.094^{* * *} \\
(0.018)\end{array}$ & $\begin{array}{c}-0.091^{* * *} \\
(0.026)\end{array}$ & $\begin{array}{c}0.031 \\
(0.043)\end{array}$ & $\begin{array}{c}-0.663^{* * *} \\
(0.221)\end{array}$ & $\begin{array}{c}-0.114^{* * *} \\
(0.029)\end{array}$ \\
\hline $\ln \left(\right.$ Trade $\left._{i, t}\right)$ & $\begin{array}{c}\beta_{2} \\
\text { (std error) }\end{array}$ & $\begin{array}{c}0.458^{* * *} \\
(0.023)\end{array}$ & $\begin{array}{c}0.721^{* * *} \\
(0.052)\end{array}$ & $\begin{array}{c}0.675^{* * *} \\
(0.116)\end{array}$ & $\begin{array}{c}0.217^{* * *} \\
(0.029)\end{array}$ & $\begin{array}{c}0.805^{* * *} \\
(0.037)\end{array}$ & $\begin{array}{c}1.345^{* * *} \\
(0.054)\end{array}$ & $\begin{array}{c}1.245^{* * *} \\
(0.227)\end{array}$ & $\begin{array}{c}0.717^{* * *} \\
(0.043)\end{array}$ \\
\hline $\ln \left(P C G D P_{i, t}\right)$ & $\begin{array}{c}\beta_{3} \\
\text { (std error) }\end{array}$ & $\begin{array}{c}0.111^{* * *} \\
(0.018)\end{array}$ & $\begin{array}{c}0.312^{* * *} \\
(0.035)\end{array}$ & $\begin{array}{c}0.828^{* * *} \\
(0.088)\end{array}$ & $\begin{array}{c}0.018 \\
(0.023)\end{array}$ & $\begin{array}{l}-0.076^{*} \\
(0.041)\end{array}$ & $\begin{array}{c}0.178^{* * *} \\
(0.058)\end{array}$ & $\begin{array}{l}-0.322 \\
(0.252)\end{array}$ & $\begin{array}{c}-0.107^{* *} \\
(0.048)\end{array}$ \\
\hline $\ln \left(T R M I_{i, t}\right)$ & $\begin{array}{c}\beta_{4} \\
\text { (std error) }\end{array}$ & $\begin{array}{l}-0.010 \\
(0.024)\end{array}$ & $\begin{array}{l}-0.046 \\
(0.037)\end{array}$ & $\begin{array}{c}0.442^{* * *} \\
(0.086)\end{array}$ & $\begin{array}{c}-0.168^{* * *} \\
(0.028)\end{array}$ & $\begin{array}{c}-0.060^{* * *} \\
(0.016)\end{array}$ & $\begin{array}{c}0.021 \\
(0.027)\end{array}$ & $\begin{array}{l}-0.136 \\
(0.099)\end{array}$ & $\begin{array}{c}-0.132^{* * *} \\
(0.018)\end{array}$ \\
\hline Constant & & $\begin{array}{c}1.508^{* * *} \\
(0.199)\end{array}$ & $\begin{array}{c}-1.839^{* * *} \\
(0.496)\end{array}$ & $\begin{array}{c}-8.957^{* * *} \\
(1.051)\end{array}$ & $\begin{array}{c}3.254^{* * *} \\
(0.299)\end{array}$ & $\begin{array}{c}1.443^{* * *} \\
(0.337)\end{array}$ & $\begin{array}{c}-3.490^{* * *} \\
(0.442)\end{array}$ & $\begin{array}{c}-4.840^{* *} \\
(2.025)\end{array}$ & $\begin{array}{c}1.632^{* * *} \\
(0.389)\end{array}$ \\
\hline Obs. & & 1,266 & 1,255 & 780 & 1,266 & 807 & 784 & 335 & 807 \\
\hline Adj. R-squares & & 0.436 & 0.327 & 0.198 & 0.276 & 0.647 & 0.563 & 0.0865 & 0.583 \\
\hline
\end{tabular}


Table 1.6: De-Facto Conditional to De-jure and Domestic Factors, LSDV ( $\mathrm{T}=35)$ (LSDV, time- and country-fixed effects, robust standard errors)

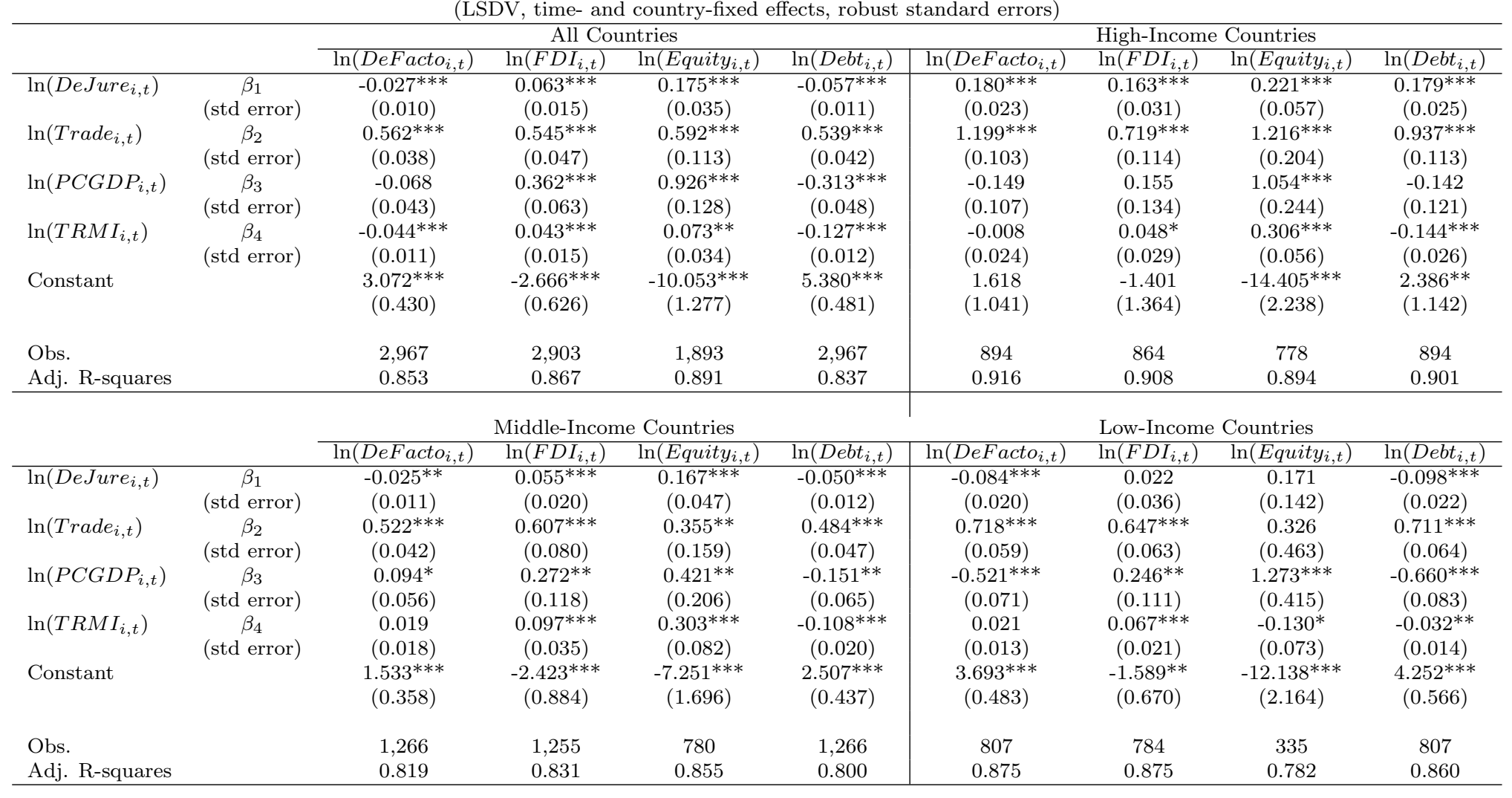


Table 1.7: De-Facto Conditional to De-jure and Domestic Factors, LSDV ( $\mathrm{T}=7$ ) (LSDV, time- and country-fixed effects, robust standard errors)

\begin{tabular}{|c|c|c|c|c|c|c|c|c|c|}
\hline & & \multicolumn{4}{|c|}{ All Countries } & \multicolumn{4}{|c|}{ High-Income Countries } \\
\hline & & $\ln \left(\right.$ DeFacto $\left._{i, t}\right)$ & $\ln \left(F D I_{i, t}\right)$ & $\ln \left(\right.$ Equity $\left._{i, t}\right)$ & $\ln \left(\right.$ Debt $\left._{i, t}\right)$ & $\ln \left(\right.$ DeFacto $\left._{i, t}\right)$ & $\ln \left(F D I_{i, t}\right)$ & $\ln \left(\right.$ Equity $\left._{i, t}\right)$ & $\ln \left(\right.$ Debt $\left._{i, t}\right)$ \\
\hline $\ln \left(\right.$ DeJure $\left._{i, t}\right)$ & $\begin{array}{c}\beta_{1} \\
\text { (std error) }\end{array}$ & $\begin{array}{l}-0.024 \\
(0.026)\end{array}$ & $\begin{array}{c}0.138^{* * *} \\
(0.047)\end{array}$ & $\begin{array}{c}0.324^{* * *} \\
(0.109)\end{array}$ & $\begin{array}{c}-0.069^{* *} \\
(0.029)\end{array}$ & $\begin{array}{c}0.269^{* * *} \\
(0.073)\end{array}$ & $\begin{array}{c}0.258^{* * *} \\
(0.095)\end{array}$ & $\begin{array}{l}0.263^{* *} \\
(0.128)\end{array}$ & $\begin{array}{c}0.277^{* * * *} \\
(0.083)\end{array}$ \\
\hline $\ln \left(\right.$ Trade $\left._{i, t}\right)$ & $\begin{array}{c}\beta_{2} \\
\text { (std error) }\end{array}$ & $\begin{array}{c}0.515^{* * *} \\
(0.079)\end{array}$ & $\begin{array}{c}0.645^{* * *} \\
(0.116)\end{array}$ & $\begin{array}{c}0.345 \\
(0.287)\end{array}$ & $\begin{array}{c}0.502^{* * *} \\
(0.093)\end{array}$ & $\begin{array}{c}1.377^{* * *} \\
(0.258)\end{array}$ & $\begin{array}{c}0.834^{* * *} \\
(0.304)\end{array}$ & $\begin{array}{l}1.135^{* *} \\
(0.567)\end{array}$ & $\begin{array}{c}1.049 * * * \\
(0.300)\end{array}$ \\
\hline $\ln \left(P C G D P_{i, t}\right)$ & $\begin{array}{c}\beta_{3} \\
\text { (std error) }\end{array}$ & $\begin{array}{l}-0.035 \\
(0.100)\end{array}$ & $\begin{array}{c}0.334^{* *} \\
(0.153)\end{array}$ & $\begin{array}{c}0.820^{* * *} \\
(0.311)\end{array}$ & $\begin{array}{c}-0.295^{* * *} \\
(0.112)\end{array}$ & $\begin{array}{l}-0.265 \\
(0.239)\end{array}$ & $\begin{array}{c}0.183 \\
(0.247)\end{array}$ & $\begin{array}{l}1.121^{*} \\
(0.647)\end{array}$ & $\begin{array}{l}-0.260 \\
(0.288)\end{array}$ \\
\hline $\ln \left(T R M I_{i, t}\right)$ & $\begin{array}{c}\beta_{4} \\
\text { (std error) }\end{array}$ & $\begin{array}{c}-0.078^{* * *} \\
(0.027)\end{array}$ & $\begin{array}{c}0.034 \\
(0.038)\end{array}$ & $\begin{array}{c}0.214^{* *} \\
(0.100)\end{array}$ & $\begin{array}{c}-0.164^{* * *} \\
(0.030)\end{array}$ & $\begin{array}{l}-0.005 \\
(0.058)\end{array}$ & $\begin{array}{c}0.062 \\
(0.074)\end{array}$ & $\begin{array}{c}0.431^{* * *} \\
(0.156)\end{array}$ & $\begin{array}{c}-0.158^{* *} \\
(0.064)\end{array}$ \\
\hline Constant & & $\begin{array}{c}2.991^{* * *} \\
(0.963)\end{array}$ & $\begin{array}{c}-2.911^{* *} \\
(1.473)\end{array}$ & $\begin{array}{c}-10.160^{* * *} \\
(3.004)\end{array}$ & $\begin{array}{c}5.485^{* * *} \\
(1.076)\end{array}$ & $\begin{array}{c}2.127 \\
(2.200)\end{array}$ & $\begin{array}{l}-2.405 \\
(2.438)\end{array}$ & $\begin{array}{c}-16.115^{* *} \\
(6.284)\end{array}$ & $\begin{array}{c}3.288 \\
(2.575)\end{array}$ \\
\hline \multirow[t]{3}{*}{$\begin{array}{l}\text { Obs. } \\
\text { Adj. R-squares }\end{array}$} & & $\begin{array}{c}646 \\
0.850 \\
\end{array}$ & $\begin{array}{c}631 \\
0.829 \\
\end{array}$ & $\begin{array}{c}425 \\
0.887 \\
\end{array}$ & $\begin{array}{c}646 \\
0.834 \\
\end{array}$ & $\begin{array}{c}185 \\
0.915\end{array}$ & $\begin{array}{c}179 \\
0.904 \\
\end{array}$ & $\begin{array}{c}163 \\
0.877\end{array}$ & $\begin{array}{c}185 \\
0.895\end{array}$ \\
\hline & & \multicolumn{4}{|c|}{ Middle-Income Countries } & \multicolumn{4}{|c|}{ Low-Income Countries } \\
\hline & & $\ln \left(\right.$ DeFacto $\left._{i, t}\right)$ & $\ln \left(F D I_{i, t}\right)$ & $\ln \left(\right.$ Equity $\left._{i, t}\right)$ & $\ln \left(\right.$ Debt $\left._{i, t}\right)$ & $\ln \left(\right.$ DeFacto $\left._{i, t}\right)$ & $\ln \left(F D I_{i, t}\right)$ & $\ln \left(\right.$ Equity $\left._{i, t}\right)$ & $\ln \left(\right.$ Debt $\left._{i, t}\right)$ \\
\hline $\ln \left(\right.$ DeJure $\left._{i, t}\right)$ & $\begin{array}{c}\beta_{1} \\
\text { (std error) }\end{array}$ & $\begin{array}{l}-0.021 \\
(0.030)\end{array}$ & $\begin{array}{c}0.143^{* *} \\
(0.062)\end{array}$ & $\begin{array}{c}0.412^{* * *} \\
(0.145)\end{array}$ & $\begin{array}{c}-0.066^{*} \\
(0.034)\end{array}$ & $\begin{array}{c}-0.123^{* *} \\
(0.049)\end{array}$ & $\begin{array}{c}0.046 \\
(0.099)\end{array}$ & $\begin{array}{c}0.429 \\
(0.415)\end{array}$ & $\begin{array}{c}-0.146^{* * * *} \\
(0.054)\end{array}$ \\
\hline $\ln \left(\right.$ Trade $\left._{i, t}\right)$ & $\begin{array}{c}\beta_{2} \\
\text { (std error) }\end{array}$ & $\begin{array}{c}0.404^{* * *} \\
(0.089)\end{array}$ & $\begin{array}{c}0.740^{* * *} \\
(0.189)\end{array}$ & $\begin{array}{l}-0.060 \\
(0.406)\end{array}$ & $\begin{array}{c}0.338^{* * *} \\
(0.102)\end{array}$ & $\begin{array}{c}0.736^{* * *} \\
(0.109)\end{array}$ & $\begin{array}{c}0.755^{* * *} \\
(0.153)\end{array}$ & $\begin{array}{c}1.172 \\
(1.065)\end{array}$ & $\begin{array}{c}0.749 * * * \\
(0.127)\end{array}$ \\
\hline $\ln \left(P C G D P_{i, t}\right)$ & $\begin{array}{c}\beta_{3} \\
\text { (std error) }\end{array}$ & $\begin{array}{c}0.269^{* *} \\
(0.108)\end{array}$ & $\begin{array}{c}0.272 \\
(0.296)\end{array}$ & $\begin{array}{c}0.401 \\
(0.391)\end{array}$ & $\begin{array}{c}0.034 \\
(0.123)\end{array}$ & $\begin{array}{c}-0.462^{* * *} \\
(0.158)\end{array}$ & $\begin{array}{c}0.172 \\
(0.278)\end{array}$ & $\begin{array}{c}1.744 \\
(1.442)\end{array}$ & $\begin{array}{c}-0.553^{* * *} \\
(0.183)\end{array}$ \\
\hline $\ln \left(T R M I_{i, t}\right)$ & $\begin{array}{c}\beta_{4} \\
\text { (std error) }\end{array}$ & $\begin{array}{l}-0.022 \\
(0.048)\end{array}$ & $\begin{array}{c}0.082 \\
(0.087)\end{array}$ & $\begin{array}{c}0.818^{* * *} \\
(0.248)\end{array}$ & $\begin{array}{c}-0.175 * * * \\
(0.055)\end{array}$ & $\begin{array}{l}-0.001 \\
(0.032)\end{array}$ & $\begin{array}{c}0.094 \\
(0.057)\end{array}$ & $\begin{array}{l}-0.134 \\
(0.211)\end{array}$ & $\begin{array}{l}-0.054 \\
(0.033)\end{array}$ \\
\hline Constant & & $\begin{array}{l}1.318^{*} \\
(0.795)\end{array}$ & $\begin{array}{l}-1.446 \\
(1.613)\end{array}$ & $\begin{array}{c}-11.207^{* * *} \\
(3.389)\end{array}$ & $\begin{array}{c}4.312^{* * *} \\
(0.969)\end{array}$ & $\begin{array}{c}2.874^{* * * *} \\
(1.005)\end{array}$ & $\begin{array}{l}-3.278^{*} \\
(1.868)\end{array}$ & $\begin{array}{c}-18.896^{* * *} \\
(5.004)\end{array}$ & $\begin{array}{c}3.072^{* * *} \\
(1.157)\end{array}$ \\
\hline Obs. & & 279 & 276 & 178 & 279 & 182 & 176 & 84 & 182 \\
\hline Adj. R-squares & & 0.815 & 0.744 & 0.881 & 0.795 & 0.887 & 0.864 & 0.785 & 0.880 \\
\hline
\end{tabular}




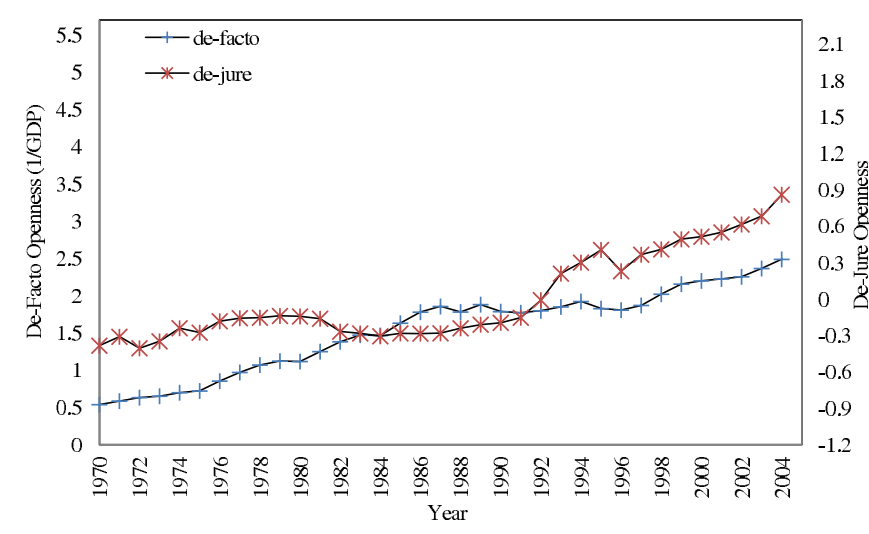

(a) All Countries.

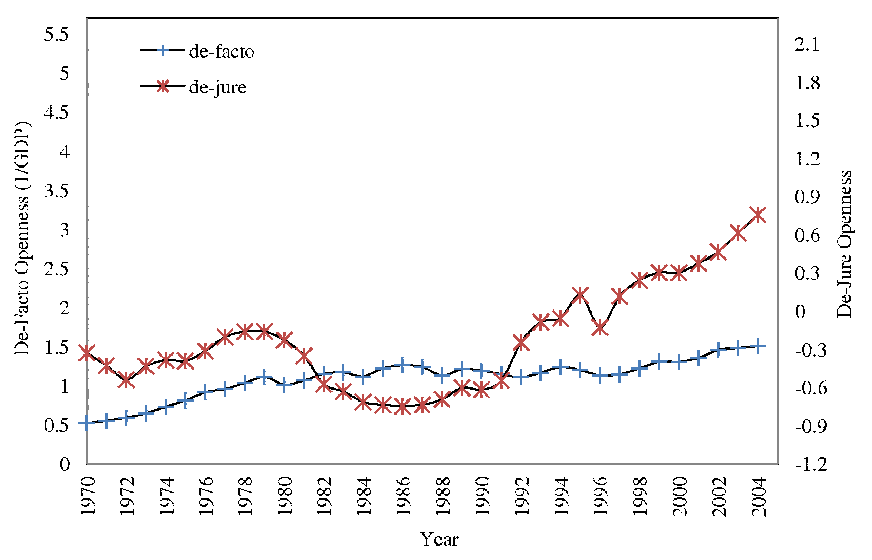

(c) Middle Income Countries.

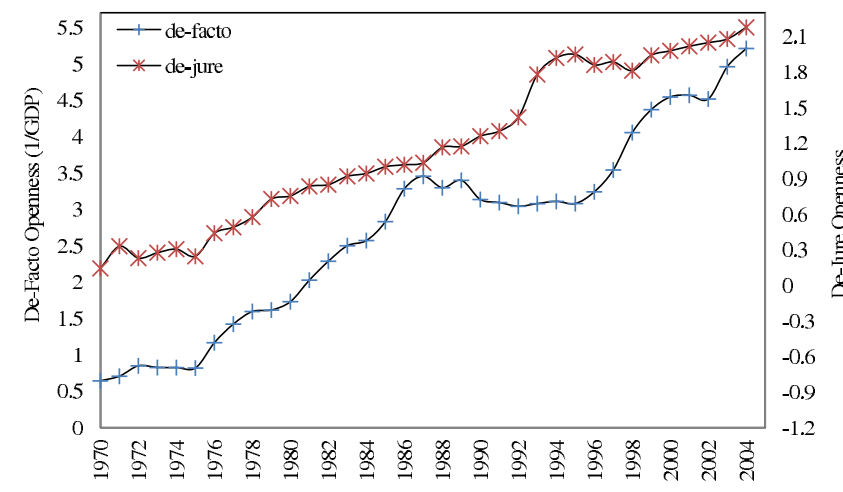

(b) High Income Countries.

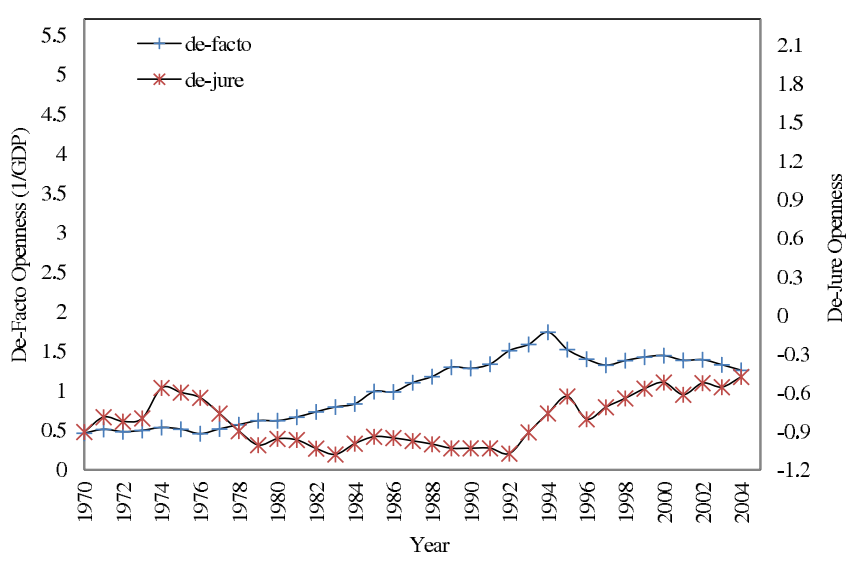

(d) Low-income Countries.

Figure 1.1: Evolution of Financial Integration: 1970-2004 


\section{Chapter 2}

\section{Determinants of Convergence in the Marginal Product of Capital}

\section{$2.1 \quad$ Introduction}

Whether or not the world capital stock is allocated proportionally to developing countries is fundamental to understanding global differences in economic development. One of the basic economic measures used to explore this question is the marginal product of capital (MPK), which is equal to capital return when production technology exhibits constant returns to scale and commodity and factor markets are perfectly competitive. Conventional wisdom suggests that the marginal product of capital converges across countries if the financial market effectively allocates worldwide production capital. Caselli and Feyrer (2007) show that MPKs do indeed converge across countries, but that convergence is conditional upon international differences in shares of reproducible capital and relative prices of final-to-investment goods.

Caselli and Feyrer's evidence regarding conditional convergence does not immediately support the conventional wisdom of a perfect financial market. The Solow growth model (Solow, 1956, 1957), for example, predicts that poor countries will catch up with rich countries in terms of capital returns and per capita gross domestic product (GDP). 
In fact, the long-run convergence process of MPK can be identical to that of economic growth (Mankiw et al., 1992; Caselli et al., 1996) if countries lack access to the global financial market. A country that opens its financial market will receive foreign capital if its capital return is higher than foreign countries' returns. The global flows of financial capital from low- to high-return countries promote MPK convergence if financial capital is transformed to reproducible capital. Although financial flows are likely to be a primary global factor, we cannot ignore the roles played by international trade and production specialization in conditional convergence. As in Stolper and Samuelson (1941) and Samuelson (1949), convergence in product prices due to freer trade causes convergence in factor prices. International trade leads countries to specialize in the production of comparative advantage goods, creating upward pressure on prices of factors used intensively in the production process. Capital returns in capital-scarce developing countries would decline because of trade liberalization.

This paper examines both domestic and international factors as potential determinants of this conditional convergence. We first develop an empirical model from Solow's growth model (Mankiw et al., 1992) and augment it to include international factors of finance and trade. We are particularly interested in comparing the contributions of domestic and international factors for MPK convergence. We use a measure of MPK from Caselli and Feyrer (2007) and develop a data set of 52 countries from 1970 to 2005. The data confirm evidence for MPK convergence over the period we consider (Caselli and Feyrer, 2007; Mello, 2009; Chatterjee and Naknoi, 2010, e.g.). The cross-country standard deviation had declined significantly from 0.048 in year 1970 to 0.028 in year 2005. We use a two-step difference GMM estimator (Arellano and Bond, 1991) and estimate the determinants for MPK convergence. Saving rates, aggregate financial flows, and international trade are found to be essential determinants of conditional convergence. Although domestic factors appear to contribute more than international factors, the results depend on econometric specifications. We also investigate the components of financial flows responsible for the findings in aggregate financial 
flows. Although FDI flows are effectively associated with MPK convergence, debt flows do not strongly reduce the global difference in MPK. One possible explanation is that financial flows without ownership do not effectively convert into recipient countries' reproducible capital stock. Finally, the literature has ignored findings in international trade. However, it is not surprising that international trade plays a role in conditional convergence. We show that international trade contributes to the convergence by equalizing relative final-to-investment prices worldwide. We believe that these results are consistent with the conflicting evidence shown in previous literature. Although financial capital, the majority of which consists of debt flows, appears not to flow strongly from low- to high-return countries (Feldstein and Horioka, 1980; Lucas, 1990), economic factors other than financial flows contribute to the conditional convergence of MPK (Mankiw et al., 1992; Caselli et al., 1996).

The remainder of this paper is organized into four sections. In Section 2, we present an empirical measure of MPK and its convergence trend. In Section 3, we develop an empirical strategy by deriving MPK from Solow's model and augmenting it with international finance and trade factors. Section 4 presents empirical results. We discuss our conclusions in the last section.

\subsection{Marginal Product of Capital}

\subsubsection{Measures of MPK}

We derive empirical measures of the marginal product of capital from the CobbDouglas production function. We assume the following: there is an aggregate production sector, there are two factor inputs, and product and factor markets are perfectly competitive. We consider the following Cobb-Douglas production function, in which output is linked to the two factor inputs of capital and effective labor: 


$$
Y_{i t}=K_{i t}^{\alpha_{i}}\left(A_{i t} L_{i t}\right)^{1-\alpha_{i}}
$$

where $Y_{i t}$ is the output of country $i$ at time $t, K_{i t}$ is the reproducible capital, $L_{i t}$ is labor, and $A_{i t}$ is labor augmenting productivity. The shares of capital and labor in national income are represented by $\alpha_{i}$ and $1-\alpha_{i}$, which are time invariant but differ across countries. Because capital in agricultural and natural-resource sectors is nonreproducible, it should not be a part of a country's physical capital stock. Following Caselli and Feyrer (2007), we use the non-reproducible capital share for $\alpha_{i}$.

To employ the constant return to scale (CRS) production technology, we make two additional assumptions. The first one states that the economy is large enough to increase the output once factor inputs are increased. The second one specifies the relative unimportance of land and other natural resources in production. 1 Under these assumptions and perfectly competitive markets of goods and factors, the compensation for one unit of physical capital $\left(r_{i t}\right)$ is equal to $M P K_{i t}$ :

$$
M P K_{i t}=\alpha_{i} \frac{Y_{i t}}{K_{i t}}
$$

Throughout this paper, we focus on presenting empirical results from this measure of MPK.2

\footnotetext{
${ }^{1}$ If natural resources were important, the production function would not be CRS since the increase in capital and labor would deliver a less than proportional increase in output.

2 Caselli and Fevrer (2007) label equation (2.1) naive, arguing that it is biased because it does not account for the difference in investment prices between rich and poor economies. However, we continue to use the measure of MPK from equation 2.1 since the price adjustment will be canceled when we solve MPK from the Solow model.
} 


\subsubsection{Data}

We compile a benchmark data set containing 52 countries 3 over a period from 1970 to 2007 . The country sample is restricted by the availability of the reproducible capital share in income $\left(\alpha_{i}\right)$. Caselli and Fevrer (2007) build an estimate of $\alpha_{i}$ from Bernanke and Gurkaynak (2002) and the World Bank.4 Since the data are available only for 1996, we assume the data to be constant over time.

To develop $M P K_{i t}$, the data on $Y_{i t}$ is derived from the Penn World Table 7.0 (Heston et al., 2011). $Y_{i t}$ is real GDP in constant 2005 international dollars. The capital stock, $K_{i t}$, is developed from the perpetual inventory method using the data on real aggregate investment:

$$
K_{i t}=(1-\delta) K_{i, t-1}+I_{i t}
$$

where $\delta$ is the depreciation rate of physical capital and $I_{i t}$ is real investment. Following Caselli and Feyrer (2007), we use the depreciation rate of $6 \%$. To obtain the initial value of the real capital stock, we compute:

$$
K_{i, 1961}=\frac{I_{i, 1961}}{\delta+\hat{g}_{i}}
$$

where $\hat{g}_{i}$ is the average growth rate of real investment in country $i$ over the period from 1961 to 1981. Although we use the real investment data starting from 1961, the capital

\footnotetext{
${ }^{3} 52$ countries are Algeria (DZA), Australia (AUS), Austria (AUT), Belgium (BEL), Bolivia (BOL), Botswana (BWA), Burundi (BDI), Canada (CAN), Chile (CHL), Colombia (COL), Congo (COG), Costa Rica (CRI), Cote d'Ivoire (CIV), Denmark (DNK), Ecuador (ECU), Egypt (EGY), El Salvador (SLV), Finland (FIN), France (FRA), Greece (GRC), Ireland (IRL), Israel (ISR), Italy (ITA), Jamaica (JAM), Japan (JPN), Jordan (JOR), Korea (KOR), Malaysia (MYS), Mauritius (MUS), Mexico (MEX), Morocco (MAR), the Netherlands (NLD), New Zealand (NZL), Norway (NOR), Panama (PAN), Paraguay (PRY), Peru (PER), Philippines (PHL), Portugal (PRT), Singapore (SGP), South Africa (ZAF), Spain (ESP), Sri Lanka (LKA), Sweden (SWE), Switzerland (CHE), Trinidad \&Tobago (TTO), Tunisia (TUN), the United Kingdom (GBR), the United States (USA), Uruguay (URY), Venezuela (VEN), and Zambia (ZMB).

${ }^{4}$ The World Bank publication 'Where is The Wealth of Nations' provides estimates of the wealth components, including natural capital.
} 
stock measures used in the empirical analysis are from 1970 to 2007.

We can deconstruct $M P K_{i t}$ from equation (2.1) into the capital share $\alpha_{i}$ and capital productivity $Y_{i t} / K_{i t}$. Because $\alpha_{i}$ is time invariant, the growth rate of MPK, $\Delta \ln \left(M P K_{i t}\right)$, is associated only with the growth rate of capital productivity. The summary statistics of MPK and capital productivity for 1970 and 2005 are reported in Table 1. In 1970, the lowest and highest values of MPK were Ecuador (0.022) and El Salvador (0.240), respectively. The difference between these two countries is roughly tenfold. Most high-income countries are in the middle range: 0.092 for France, 0.112 for the United States, and 0.136 for Japan. The MPK measures from 1970 indicate significant dispersion, deriving mainly from low-income countries. In 2005, the country with the lowest MPK value was Burundi (0.023), with El Savador continuing to hold the highest MPK value (0.164). Although the ratio of the lowest to the highest MPK remains significant (approximately sevenfold), the cross-country standard deviation declines significantly from 0.046 to 0.024 .

\subsubsection{Overview of MPK Convergence}

To understand the convergence process of MPK across countries and years, we study

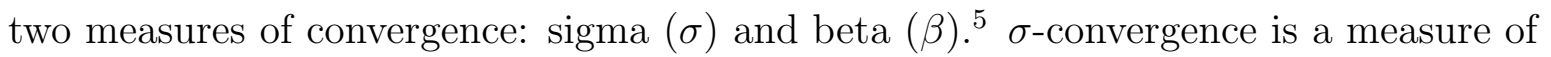
dispersion from the sample mean, which incorporates the standard deviations of $n$ countries' MPK measures in each year. If dispersion from a sample mean declines over time, there is evidence for cross-country $\sigma$-convergence. Figure 1 shows the long-run trend of dispersion in MPK from 1970 to 2007. In 1970, the standard deviation across 52 countries was the largest. There was a sharp drop over the period from 1970 to 1985, followed by a period of relative stability.

$\beta$-convergence examines the relationship between the growth rates over the period $\tau$ against the initial levels across countries. If MPK converges over time across countries,

\footnotetext{
${ }^{5}$ See Barro and Sala-i-Martin (1992) and Sala-i-Martin (1996) for the discussion on $\sigma$ - and $\beta$ convergence in neoclassical growth model.
} 
$\beta$ in the following equation would be negative:

$$
\ln \left(M P K_{i t}\right)-\ln \left(M P K_{i, t-\tau}\right)=\phi+\beta \ln \left(M P K_{i, t-\tau}\right)+\epsilon_{i}
$$

This type of convergence is referred to as catching-up convergence. Figure 2 reports $\beta$-convergence when $t=2005$ and $\tau=35$. The average growth rates in MPK are found to be negatively and statistically significantly associated with the initial values. We find that the $\beta$ coefficient is -0.51 , indicating $\beta$-type convergence: the higher the initial values, the higher the subsequent declining rates. The corresponding speed of convergence is around $2 \%$ per year. It implies that the average time an MPK spends to cover half of the distance between its initial position and its steady-state is about 36 years. Both figures show a tendency for dispersion of MPK to decline over time, suggesting the presence of a long run equalization mechanism in the marginal product of capital.

\subsection{Theory for MPK Convergence}

\subsubsection{Domestic Factors}

We wish to examine the economic factors causing the convergence in MPK. We start by considering domestic factors likely to play critical roles in MPK convergence. Our theoretical basis is built upon the Solow growth model in Mankiw et al. (1992). Here, we use Solow's model as a baseline to associate our discussion with empirical literature on conditional convergence in economic growth. The Solow model assumes that a constant fraction of output, $s_{i, t-\tau}$, is saved and invested to production process. The saving rate is defined as the share of real domestic investment to real GDP. We define $k_{i t}$ as the stock of capital per effective unit of labor: $k_{i t}=K_{i t} /\left(A_{i t} L_{i t}\right)$, and $y_{i t}$ as the level of output per effective unit of labor: $y_{i t}=Y_{i t} /\left(A_{i t} L_{i t}\right)$. The evolution of 
$k_{i t}$ is then characterized by the following equation:

$$
\Delta k_{i t}=s_{i, t-\tau} y_{i t}-\left(n_{i t}+g_{i t}+\delta\right) k_{i t}
$$

where $n_{i t}$ is the average population growth rate between $t-\tau$ to $t$, and $g_{i t}$ is the growth rate of labor productivity from $t-\tau$ to $t 6$

Equation (2.2) implies that $k_{i t}$ converges to a steady-state capital to effective labor ratio:

$$
\frac{K_{i t}}{A_{i t} L_{i t}}=\left[\frac{s_{i, t-\tau}}{n_{i t}+g_{i t}+\delta}\right]^{\frac{1}{1-\alpha_{i}}}
$$

Using equations (2.1) and (2.3) , we can derive the marginal product of capital as the following:

$$
M P K_{i t}=\alpha_{i}\left[\frac{n_{i t}+g_{i t}+\delta}{s_{i, t-\tau}}\right]
$$

Our equation (2.4) corresponds to equation (17) in Mankiw et al. (1992). The equation predicts that while the marginal product of capital would be negatively correlated with the initial saving rate $\left(s_{i, t-\tau}\right)$, it would be positively correlated with the capital share $\left(\alpha_{i}\right)$, population growth rate $\left(n_{i t}\right)$, human capital growth $\left(g_{i t}\right)$, and depreciation rate $(\delta)$. Here, we call $\left(n_{i t}+g_{i t}+\delta\right)$ an effective depreciation rate. This equation

\footnotetext{
${ }^{6}$ To obtain labor-augmenting productivity, we first derive it from Cobb-Douglas production function:

$$
\ln \left(A_{i t}\right)=\frac{\ln \left(Y_{i t}\right)-\alpha_{i} \ln \left(K_{i t}\right)-\left(1-\alpha_{i}\right) \ln \left(L_{i t}\right)}{\left(1-\alpha_{i}\right)} .
$$

We think, however, it is problematic to use $A_{i t}$ derived directly from the function above as a proxy for a measure of labor-augmenting productivity. Thus, we use the fitted values $\left(\bar{A}_{i t}\right)$ from the following equation:

$$
\ln \left(A_{i t}\right)=\gamma_{i}^{1} \ln \left(E d u_{i t}^{1}\right)+\gamma_{i}^{2} \ln \left(E d u_{i t}^{2}\right)+\gamma_{i}^{3} \ln \left(E d u_{i t}^{3}\right)+\gamma_{t}^{4}+\varepsilon_{i t}
$$

where $E d u_{i t}^{1}, E d u_{i t}^{2}$, and $E d u_{i t}^{3}$ are the average years of primary schooling, secondary schooling, and tertiary schooling, respectively, from Barro and Lee (2010). Our empirical measure of $g_{i t}$ is the growth rate of $\bar{A}_{i t}$ from year $t-\tau$ to $t$.
} 
indicates that these domestic factors would be predictors of the MPK. Taking the log to both sides of equation (2.4), we derive the following equation (2.5):

$$
\ln \left(M P K_{i t}\right)=\ln \left(\alpha_{i}\right)-\ln \left(s_{i, t-\tau}\right)+\ln \left(n_{i t}+g_{i t}+\delta\right)
$$

Finally, we rewrite equation (2.5) as a growth model in the level of the marginal product of capital similar to Caselli et al. (1996):

$$
\ln \left(M P K_{i t}\right)=\beta_{1} \ln \left(M P K_{i, t-\tau}\right)+\beta_{2} \ln \left(\alpha_{i}\right)+\beta_{3} \ln \left(s_{i, t-\tau}\right)+\beta_{4} \ln \left(n_{i t}+g_{i t}+\delta\right)+\epsilon_{i t}
$$

In equation (2.6), we expect that $\beta_{1}$ is in the range between 0 and 1 . A value of $\beta_{1}$ closer to zero indicates a greater tendency towards convergence because the average growth rate, $\ln \left(M P K_{i, t}\right)-\ln \left(M P K_{i, t-\tau}\right)$, is inversely related to $\ln \left(M P K_{i, t-\tau}\right)$. Here, $\left(\beta_{1}-1\right)=-\left(1-e^{-\lambda \tau}\right)$ should be negative, implying that a country relatively close to its steady-state MPK value experiences a slowdown in the evolution of MPK. Note that $\lambda$ is the convergence rate and the estimate of the speed at which a country's MPK converges toward its steady state value.

To seek the potential impact on the conditional convergence of a change in the key domestic factor (saving rates), we develop a standard deviation in saving rates across 52 countries for each year. Figure 3 reports $\sigma$-convergence of saving rates over 38 years. Interestingly, saving rates diverge initially and converge significantly after 1982 . The decrease in the cross-country variation is partially due to the increase in saving rates in developing economies 7 An increase in saving rate implies an increase in capital per worker. Following Solow's model and discussion by Mankiw et al. (1992), this should drive capital return down. Convergence in saving rates would result from the reduction

\footnotetext{
'Loayza et al. (2000) examine a large cross-country and time-series data on national saving. They find that, on average, gross national saving rate in industrial countries has declined persistently after 1973. The evidence on developing countries is confounding: saving rates in East and South Asia countries rise, while saving rates in Latin America, the Caribbean, North and Sub-Saharan Africa regions decline.
} 
of barriers to capital mobility. In this case, a change in saving rate in one country would spread across all countries, causing capital returns to equalize across these countries (Feldstein and Horioka, 1980).

\subsubsection{International Flows of Financial Assets}

We now turn to the global factors that may cause convergence in MPK. In particular, international financial flow is believed to be the primary factor that equalizes capital returns across countries. In the absence of barriers to capital mobility, capital owners seek more productive opportunities in countries with a high return of capital until capital returns are equalized worldwide. There could be several reasons that prevent financial flows from flowing into the countries with high returns of capital. While Lucas (1990) notes the presence of credit-frictions, Caselli and Fevrer (2007) argue that the high costs of installing capital in poor countries prevent financial capital from flowing to them.

We add a measure of international financial flows to the main equation (2.6) to examine whether financial flows, potentially transformed into reproducible capital, influence MPK convergence. We construct a ratio of net cross-border financial flows to nominal GDP. Financial flows are the aggregate of portfolio equity, FDI, debt, and other financial flows from Lane and Milesi-Ferretti (2007) 8

Our baseline model is augmented with a measure of net financial flows, $\frac{N e t F l o w_{i, t-5}}{G D P_{i, t-5}}$ :

\footnotetext{
8 Lane and Milesi-Ferretti (2007) provide details on data definitions as well as data development strategy. International portfolio flows refer to investors' purchase of issued equity containing countryfunds, depository receipts, and direct purchases of shares. Portfolio equity holdings include direct purchases of shares in local companies and mutual funds that are below $10 \%$ of ownership level. Foreign direct investment (FDI) includes direct investment in a local company to control stakes (above $10 \%$ of ownership level) as well as green field investment. The debt category includes debt securities, bank loans, deposits, and other debt instruments. Other flows include financial derivatives and total reserves minus gold.
} 


$$
\begin{aligned}
\ln \left(M P K_{i t}\right)= & \beta_{1} \ln \left(M P K_{i, t-5}\right)+\beta_{2} \ln \left(\alpha_{i}\right)+\beta_{3} \ln \left(s_{i, t-5}\right) \\
& +\beta_{4} \ln \left(n_{i t}+g_{i t}+\delta\right)+\beta_{5}\left(\text { NetFlow }_{i, t-5} / G D P_{i, t-5}\right)+\epsilon_{i t}
\end{aligned}
$$

We expect negative relationship between the net financial flows and the MPKs, as more financial capital in a given country (transformed to the reproducible capital) should reduce the MPK.

\subsubsection{International Trade}

While financial flows are likely to be a primary global cause of convergence, we cannot ignore the role of international trade and production specialization. As in Stolper and Samuelson (1941) and Samuelson (1949), convergence in product prices due to freer trade causes convergence in factor prices. This concept of factor price equalization is a well-known theory of international trade.9 International trade causes countries to specialize in producing their comparative advantage goods, creating upward (downward) pressure on the prices of factors that are used heavily (lightly) in production process. It is intuitively convenient to consider international flows of products to be embodied factor input flows. For example, a capital-abundant country (Japan) exports capital-intensive goods (machinery) to a capital-scarce country (Vietnam) and imports labor-intensive goods (textiles) from Vietnam. Because machinery production requires more capital and less labor than textile production, Japanese machinery exports embody more capital and less labor than Japanese textile imports do. In other words, Japan is a net exporter and Vietnam is a net importer of embodied capital.

\footnotetext{
${ }^{9}$ The empirical study of international trade has rejected the FPE theory. See Davis and Weinstein (2001), who argue that the breakdown of the FPE theory is essential to supporting global production and trade data. However, Nishioka (2012) finds that the empirical rejection of the FPE theory in Davis and Weinstein (2001) stems not from capital but from labor. The industry-level measures of capital productivity, a main component of MPK, are similar across countries and industries from the 2000 data.
} 
Thus, production specialization causes capital return in Japan to increase and that in Vietnam to decline, creating the equalization force in capital returns.

International trade arises from global differences in product price. Vietnam has an incentive to purchase machinery from Japan because Japanese machinery is cheaper than its Vietnamese counterpart. The intuition behind international trade as a cause of MPK convergence stems from the declining trend in the log of the relative price: $\ln \left(P_{i t}^{y} / P_{i t}^{k}\right) .10$ Figure 4 provides insight on how international trade can affect MPK convergence. The figure depicts $\sigma$-convergence in the price ratios of final-to-investment goods for each year and provides evidence that the relative prices are equalized across countries.

To examine how physical capital embodied in international trade influences returns to capital across countries, we introduce a measure of capital content of trade according to Leamer (2000). Our measure is consistent with the Heckscher-Ohlin-Vanek (HOV) model Vanek (1968) and is derived from the following equation:11

$$
K C T_{i t}=K C E_{i t}-K C I_{i t}=E X_{i t} \frac{K_{i t}}{G D P_{i t}}-\sum_{j \in C_{52}} I M_{i j t} \frac{K_{j t}}{G D P_{j t}}
$$

where $C_{52}$ is the subset of 52 countries, $E X_{i t}$ is the export value (nominal $\$$ US) of country $i$ to the subset of 52 countries, $G D P_{i t}$ is the gross domestic product (nominal $\$ \mathrm{US})$ of country $i$, and $I M_{i j t}$ is the bilateral import of country $i$ from country $j$.

\footnotetext{
${ }^{10} P_{i t}^{y}$, and $P_{i t}^{k}$ are derived from the Penn World Table 7.0 (Heston et al., 2011). $P_{i t}^{y}$ is the price level of gross domestic product (PPP over GDP), and $P_{i t}^{k}$ is the price level of investment (PPP over investment). See also Hsieh and Klenow (2007) who find that the price of investment goods relative to consumptions goods tends to be higher in richer countries.

${ }^{11}$ Typically, the empirical literature on the HOV model employs data from Input-Output Tables because the primary objective of the HOV model is to examine factor trade arising from industry composition. However, we develop the measure of capital content of trade similarly to the measure of R\&D content of trade proposed by Lichtenberg and v. Pottelsberghe de la Potterie (1998). We use country-specific capital intensity to estimate capital content of trade, omitting capital content of trade arising from industry composition. Because the subject of this paper is the long-run effect of capital content of trade for MPK convergence, and because Input-Output Tables are not available for timeseries data, we use the measure used by Lichtenberg and v. Pottelsberghe de la Potterie (1998). This measure is also similar to Davis and Weinstein (2001) because there is a small variation in industrylevel measures of $K_{i t} / Y_{i t}$ within a country but considerable variation across countries (Nishioka, 2011).
} 
The HOV model predicts that a capital abundant country is an exporter of embodied capital, and a capital scarce country is an importer of embodied capital. In other words, a country that exports capital content more than its imports is a net exporter of capital with a positive measure of $K C T_{i t}$. In our study, the data on bilateral exports and imports are obtained from the United Nations Commodity Trade Statistics Database and the Direction of Trade Statistics (IMF) 12

Our model is augmented to include an additional measure: the capital content of trade divided by the domestic capital stock $\left(K C T_{i, t-5} / K_{i, t-5}\right)$ :

$$
\begin{aligned}
\ln \left(M P K_{i t}\right)= & \beta_{1} \ln \left(M P K_{i, t-5}\right)+\beta_{2} \ln \left(\alpha_{i}\right)+\beta_{3} \ln \left(s_{i, t-5}\right) \\
& +\beta_{4} \ln \left(n_{i t}+g_{i t}+\delta\right)+\beta_{6}\left(K C T_{i, t-5} / K_{i, t-5}\right)+\epsilon_{i t}
\end{aligned}
$$

We expect a negative relationship between the capital content of trade and the MPK. A capital abundant country is an exporter of $K C T_{i t}$, and the return to capital is low in a capital abundant country. Figures 2.5 and 2.6 exhibit the scatter plots of $K C T_{i t} / K_{i t}$ against $M P K_{i t}$ for 1970 and 2005, respectively. In 1970, there is a rough but negative association between these two measures. The measures of MPK for net importers of embodied capital are higher than those for net exporters of embodied capital. However, the negative relationship is weaker in 2005. The returns to capital are clustered together within a narrow range, indicating that both measures had equalized significantly over the period.

\footnotetext{
${ }^{12}$ We use nominal values (\$US) for exports, imports, and GDP to measure capital content of trade. We use previously developed measure of capital stock. Because the bilateral trade values of exports and imports are not available for some years of Botswana (1970-1995) and South Africa (1970-1995), we use the values from the closest year (2000) to interpolate the data. For example, the unavailable bilateral imports of year 1995 for Botswana are estimated from the total imports in year 1995 and bilateral import shares from year 2000 .
} 


\subsection{Empirical Evidence}

\subsubsection{Baseline Results}

To study the convergence mechanism of the marginal product of capital, we use equation (2.6) as a baseline and augment it to include international factors. We are particularly interested in financial capital flows as in equation (2.7) and embodied capital flows as in equation (2.8). The data set is an unbalanced panel consisting of at most 364 observations from 52 countries at 5-year intervals $(\tau=5)$ during the period from 1970 to 2005.

Equation (2.6) represents a dynamic model with a lagged-dependent variable on the right-hand-side, which gives rise to autocorrelation and other econometric problems typical of dynamic time-series panel data. In particular, it is critical to account for endogeneity problems to obtain consistent estimators from equation (2.6). To avoid this problem, Caselli et al. (1996) introduce the difference generalized method of moments (difference GMM, Arellano and Bond (1991)) and estimate a cross-country convergence rate of economic growth. We follow Caselli et al. (1996) and employ difference GMM as our preferred estimator. It is a good fit for our study because we have a short time-span $(T=8)$ and a large cross-section $(N=52) .13$ We use the two-step estimator instead of the one-step because it is asymptotically efficient and robust to panel-specific autocorrelation, different patterns of heteroskedasticity, and cross-country correlation (Bond et al., 2001).

Nevertheless, it is worthwhile to start estimation of the dynamic equation with ordinary least squares (OLS). Although the estimators from OLS are biased, the results provide us with the rough ranges of the true parameters. The problem with applying

\footnotetext{
${ }^{13}$ We prefer difference GMM over system GMM. System GMM for our purpose has limitations. First, only variables that do not correlate with country fixed effects can be included in the model. Second, system GMM assumes the larger number of instruments than difference GMM do. This limits the choices for instruments. Finally, in the case when the number of instruments is greater than the number of countries, the instruments and test for overidentifying restrictions could be weak.
} 
OLS is that the lagged dependent variable is positively correlated with the fixed effects in the error term, which gives rise to dynamic panel bias. The coefficient for the lagged dependent variable $\left(\beta_{1}\right)$ is upward biased because it captures the power that is supposed to belong to country fixed effects (Hsiao, 1986). This bias results in a downward bias in the estimate of the speed of convergence: $\lambda$ (Caselli et al., 1996).

We start our estimation with a simple conditional convergence model that includes only a lagged dependent variable, denoted as Model 1. Then, Model 2 adds domestic factors, as shown in equation (2.6). Next, we estimate equation (2.7) as Model 3 and equation (2.8) as Model 4. Finally, Model 5 augments equation (2.6) by including both financial and embodied capital flows. We include time dummy variables that have the same effect as transforming the variables into deviations from time means. They remove universal time-related shocks from errors, preventing any sort of cross-country and contemporaneous correlations.

Estimation results from OLS are reported in Table $(2.2(\mathrm{a}))$. The coefficient on the initial level of the marginal product of capital $\left(\beta_{1}\right)$ in Model 1 is close to one (0.929), implying that the speed of convergence $(\lambda)$ is slow (1.5\%). In Model $2, \beta_{1}$ declines from 0.929 to 0.679 and the speed of convergence $(\lambda)$ increases from $1.5 \%$ to $7.7 \%$, indicating the importance of domestic factors for conditional convergence. The values of $\beta_{1}$ remain stable in the subsequent models. The coefficient on the initial level of capital share is positively and statistically significantly associated with MPK as the theory suggests, which confirms that MPK convergence is 'conditional' upon countryspecific capital shares (Caselli and Feyrer, 2007). The saving rate $\left(s_{i, t-5}\right)$ and effective depreciation rate $\left(n_{i t}+g_{i t}+\delta\right)$ have opposite effects in signs. While a $1 \%$ increase in the initial saving rate leads to a $0.21 \%$ decrease in MPK, a $1 \%$ increase in the effective depreciation rate leads to a $0.10 \%$ increase in MPK. Models 3, 4, and 5 introduce financial and trade flows. However, only financial flows appear to be correlated with MPK. Overall, the OLS results indicate that the inclusion of domestic factors improves 
the fit of the regression 14 although the results are subject to econometric problems, such as endogeneity.

Next, we estimate the five models with the least squares dummy variables (LSDV), including time- and country-specific dummy variables. Here we need to drop the timeinvariant and country-specific capital share $\ln \left(\alpha_{i}\right)$ from the equations 15 The LSDV estimators do not eliminate dynamic panel bias because the estimators are only consistent when all right-hand side variables are strictly exogenous. The results from LSDV estimations are reported in Table $(2.2(\mathrm{~b}))$. As in Model $1, \beta_{1}$ is 0.619 . In comparison to the corresponding OLS result, the speed of convergence increases significantly from $1.5 \%$ to $9.6 \%$ when we include country-specific fixed effects. MPK convergence is conditional for country-specific factors, which may include time-invariant reproducible capital share. Across all models, the coefficients on saving rates $\left(\beta_{3}\right)$ and effective depreciation rates $\left(\beta_{4}\right)$ are statistically significant with MPK. However, since our empirical measures of $\ln \left(n_{i t}+g_{i t}+\delta\right)$ are relatively stable over time, country-specific dummy variables may capture the cross-country variation in $\ln \left(n_{i t}+g_{i t}+\delta\right)$. The coefficient of capital content of trade in Model 4 is insignificant but exhibits a greater value than the corresponding OLS coefficient. When all domestic and global factors are included as in Model 5 , the coefficient of international trade is negative but statistically insignificant. The financial flow variable is significant in both Models 3 and 5.16

Although the results from OLS and LSDV provide insight regarding the power and sign of domestic and global factors affecting MPK convergence, these results are subject to the econometric problems discussed above. Now, we employ a difference GMM

\footnotetext{
${ }^{14}$ Adjusted $R^{2}$ increases from 0.932 (Model 1) to 0.959 (Model 2). However, they do not improve when we introduce global factors (Models 3, 4, and 5). Akaike Information Criterion (AIC) and Bayesian Information Criterion (BIC) indicate that the model that includes only trade flows represents a better statistical fit (AIC and BIC are not reported).

${ }^{15}$ The country fixed effect captures time-invariant country-specific determinants of MPK, which include capital share $\left(\alpha_{i}\right)$.

${ }^{16}$ Adjusted $R^{2}$ increases from 0.950 (Model 1) to 0.963 (Model 2), however, as in the case of OLS estimates, they do not improve as we add international factors. AIC and BIC (not reported) indicate that Models 3 and 5 have the best statistical fit.
} 
estimator that suits our econometric needs 17 The difference GMM uses equation (2.6) in first-difference by transforming it into:

$$
\Delta \ln \left(M P K_{i t}\right)=\beta_{1} \Delta \ln \left(M P K_{i, t-5}\right)+\beta_{3} \Delta \ln \left(s_{i, t-5}\right)+\beta_{4} \Delta \ln \left(n_{i t}+g_{i t}+\delta\right)+\xi_{t}+\Delta \epsilon_{i t}
$$

where $\xi_{t}$ is a year-specific constant, capturing productivity changes and other factors common to all countries. Note that first-difference transformation removes countryspecific fixed effects.

We treat all right-hand side variables in the equation above as endogenous variables. It is logical to consider that domestic variables are endogenous because we derive MPK from the steady-state condition in equation (2.2). Moreover, international variables are also endogenous because financial and embodied capital flows arise simultaneously from the global difference in returns to capital. See, for example, Mundell (1957) and Antràs and Caballero (2009) for theoretical consideration of this concept. Lagged levels of endogenous regressors are used as instruments, including the first-difference lagged dependent variable. The validity of instruments is determined by the consistency of parameter estimates and specification tests: the Hansen test for overidentifying restrictions, difference-in-Hansen tests for exogeneity of instrument subsets, and the Arellano-Bond (AR) test for first- and second-order serial correlation in differences. These specification tests indicate that the moment conditions are robust p-values of $\mathrm{AR}(2)$. The Hansen and difference-Hansen statistics signal that errors are not serially correlated. $\mathrm{AR}(2)$, the test for second-order correlation in differences, is important because it detects autocorrelation in levels. We find no evidence for serial correlation in levels, suggesting that fluctuations in economic activity do not influence our results.

\footnotetext{
17 Bond et al. (2001) argue that the first-differenced GMM estimator can be a poor estimator of cross-country growth regressions due to a possible weak correlation of the lagged levels of series with the subsequent first-differences. We test the correlations and find a strong relationship between the first-differences and the second lags of the level variables. Therefore, at a minimum, the second lags of endogenous variables can serve as valid instruments for our purpose.
} 
The results of GMM estimation and its specification tests are reported in Table 2.2(c) . The coefficients on the initial level of MPK $\left(\beta_{1}\right)$ in all models are positive and statistically significant at the $1 \%$ confidence level. The speed of convergence ranges from $3.1 \%$ in Model 1 to $8.6 \%$ in Model 3. Averaging all of the models, the speed of convergence is $6.6 \%$, which is in between the average speeds from the OLS and LSDV estimations 18

We observe higher convergence rates when financial variables are included $(\lambda$ is $7.4 \%$ for Model 3 and $8.6 \%$ for Model 5). The effects of international factors are not negligible when considering the convergence process of MPK. The coefficients on saving rates, $\ln \left(S_{i, t-5}\right)$, and effective depreciation rates, $\ln \left(n_{i t}+g_{i t}+\delta\right)$, are opposite in signs with similar magnitude. Models 4 and 5 indicate that the capital content of trade is negatively associated with MPK, and its effect on convergence rates is greater relatively to the effect of financial variable 19

\subsubsection{Robustness Check}

We check for robustness of the above results by introducing alternative measures for financial and trade variables. We continue to employ a two-step difference GMM estimator. Alternative measures of financial variables are financial openness and disaggregated financial flows (FDI and debt flows). We use a measure of financial openness from Chinn and Ito (2008), who developed the country-level index of restrictions on

\footnotetext{
${ }^{18}$ The convergence speed of $6.6 \%$ per year implies that the average time a MPK spends to cover half of the distance between its initial positive and its steady-state is about ten and a half years. MPK half-life convergence time is similar to GDP per capita half-life convergence life obtained by Caselli et al. (1996) which is about seven years. The differences in per-capita income levels across countries can be related to the differences in MPK levels across countries.

${ }^{19}$ We acknowledge an alternative method that allows to obtain the contribution of each explanatory variable as proposed by Blinder (1973) and Oaxaca (1973). According to their method, the mean values of each variable are combined with the coefficients from estimated regressions to produce 'predicted values'. Then, comparing predicted and actual measures would indicate the extent to which differences in international trade or financial flows account for differences in MPK across countries. However, this method would not allows us to conclude about the effect the explanatory variables have on the MPK convergence rate across the sample.
} 
capital mobility 20 Using this measure, we can examine whether a reduction in financial restrictions explains MPK convergence. By introducing net FDI and debt financial flows separately, we can study which components of aggregate financial flow are effectively transformed into physical capital. We intend to compare our results with those in Chatterjee and Naknoi (2010), who study the frictions in the transformation of financial capital into physical capital. The data on FDI and debt flows are from Lane and Milesi-Ferretti (2007). Table 2.3 reports the estimation results for alternative financial variables. Here, we compare them with the results of Model 3 reported in Table $2.2(\mathrm{c})$,

Column 2 in Table 2.3 reports the model with a financial openness variable. The coefficient on the openness measure is negative, implying that the more a financial market is liberalized, the lower its return to capital. However, this coefficient is statistically insignificant. Column 3 introduces FDI flows. The coefficient is negative and statistically significant at the $1 \%$ confidence level, implying that FDI inflows are likely to be transformed into physical capital. The coefficient on debt flows is significant (column 4), however lower is value compared to FDI inflows, suggesting that loans or financial aids have low impact on a country's capital stock. This finding confirms the argument in Caselli and Feyrer (2007), who state that increase in aid flows in the absence of financial repression results in capital outflows. Columns 5 and 6 report the estimations of Model 3 in Table 2.2(c) at different time periods. The coefficient on total net financial flows is higher in the period from 1970 to 1995 than in the period from 1985 to 2005. The convergence exhibits higher rate before 1995. These results suggest that the influence of financial flows in the early periods is stronger than those in later periods.

\footnotetext{
${ }^{20}$ This index is developed from IMF's 'Annual Report on Exchange Arrangements and Exchange Restrictions (AREAER)' and captures the intensity of regulatory restrictions on cross-border capital flows. Specifically, it is based on the principal components of four IMF binary variables: (1) existence of multiple exchange rates, (2) restrictions on current account transactions, (3) restrictions on capital account transactions, and (4) required surrender of export proceeds. This index represents the existence of different types of financial restrictions and ranges from -2.5 to 2.5 to represent a spectrum from full control to complete liberalization. We scaled the index to avoid negative values. Note that developed countries have higher index values than developing and emerging countries.
} 
Similar to the results from the financial measures, the results for trade variable from the period from 1970 to 1995 support stronger relationship between embodied capital flows and MPK measures.

Alternative measures of international trade include the log of capital content of imports $\left(K C I_{i t}\right)$ and the trade openness index. We develop the trade openness index from the sum of a country's total imports and exports divided by its GDP. This index roughly measures the level of trade openness. Table 2.4 reports the results. The coefficients on lagged dependent variables and domestic factors are statistically significant and have expected signs in all models. In column 2, the coefficient on the log of import capital content is negative and statistically significant at $1 \%$ confidence level. Higher imports of capital intensive goods has a downward pressure on domestic MPK. Column 3 reports the results when using the trade openness index. We find a negative and statistically significant coefficient at the $1 \%$ confidence level. A country with greater openness for trade has a lower MPK value.

\subsection{Conclusion}

This paper examined the economic factors that drive convergence in the marginal product of capital. We developed our empirical model from Solow's growth model (Mankiw et al., 1992) and augmented it to include international factors of finance and trade. We used a measure of MPK from Caselli and Feyrer (2007) and developed a data set of 52 countries from 1970 to 2005. Using two-step difference GMM estimators (Arellano and Bond, 1991), we found that saving rates, FDI flows, and trade flows are key determinants of conditional convergence of MPK. We found no strong evidence that debt financial flows reduce the global difference in MPK. One possible explanation of this result is that financial flows without ownership may not be transformed to reproducible capital stock. In fact, international trade, measured by capital content of trade, contributes to the convergence by equalizing international prices of investment 
and consumption goods. 


\subsection{Tables and Figures}

Table 2.1: Summary Statistics

\begin{tabular}{lccclcl}
\hline & Mean & Std. dev & \multicolumn{2}{c}{ Min value } & \multicolumn{2}{c}{ Max value } \\
\hline Year: 1970 & & & & & & \\
MPK & 0.092 & 0.046 & 0.022 & (Ecuador) & 0.240 & (El Salvador) \\
Capital productivity & 0.535 & 0.265 & 0.186 & (Tunisia) & 1.896 & (Burundi) \\
$\quad$ Year: 2005 & & & & & & \\
MPK & 0.073 & 0.024 & 0.023 & (Burundi) & 0.1645 & (El Salvador) \\
Capital productivity & 0.432 & 0.144 & 0.2132 & (Jordan) & 1.031 & (Ivory coast) \\
\hline
\end{tabular}


Table 2.2: MPK Convergence Conditional to Domestic and International Factors (a) OLS (ordinary least squares, time-fixed effects, robust standard errors)

\begin{tabular}{|c|c|c|c|c|c|c|}
\hline & & Model 1 & Model 2 & Model 3 & Model 4 & Model 5 \\
\hline Convergence rate & $\lambda$ & $1.5 \%$ & $7.7 \%$ & $7.1 \%$ & $7.7 \%$ & $7.2 \%$ \\
\hline $\ln \left(M P K_{i, t-5}\right)$ & $\begin{array}{c}\beta_{1} \\
\text { (std error) }\end{array}$ & $\begin{array}{c}0.929^{* * *} \\
(0.017)\end{array}$ & $\begin{array}{c}0.679^{* * *} \\
(0.022)\end{array}$ & $\begin{array}{c}0.700^{* * *} \\
(0.024)\end{array}$ & $\begin{array}{c}0.681^{* * *} \\
(0.026)\end{array}$ & $\begin{array}{c}0.696^{* * *} \\
(0.027)\end{array}$ \\
\hline $\ln \left(\alpha_{i-5}\right)$ & $\begin{array}{c}\beta_{2} \\
\text { (std error) }\end{array}$ & & $\begin{array}{c}0.313^{* * *} \\
(0.027)\end{array}$ & $\begin{array}{c}0.300 * * * \\
(0.032)\end{array}$ & $\begin{array}{c}0.311^{* * *} \\
(0.030)\end{array}$ & $\begin{array}{c}0.305^{* * *} \\
(0.034)\end{array}$ \\
\hline $\ln \left(s_{i, t-5}\right)$ & $\begin{array}{c}\beta_{3} \\
\text { (std error) }\end{array}$ & & $\begin{array}{c}-0.215^{* * *} \\
(0.026)\end{array}$ & $\begin{array}{c}-0.200^{* * *} \\
(0.027)\end{array}$ & $\begin{array}{c}-0.214^{* * *} \\
(0.026)\end{array}$ & $\begin{array}{c}-0.201^{* * * *} \\
(0.027)\end{array}$ \\
\hline $\ln \left(n_{i t}+g_{i t}+\delta\right)$ & $\begin{array}{c}\beta_{4} \\
\text { (std error) }\end{array}$ & & $\begin{array}{c}0.100 * * * \\
(0.023)\end{array}$ & $\begin{array}{c}0.096 * * * \\
(0.024)\end{array}$ & $\begin{array}{c}0.100 * * * \\
(0.023)\end{array}$ & $\begin{array}{c}0.097 * * * \\
(0.024)\end{array}$ \\
\hline NetFlow $_{i, t-5} / G D P_{i, t-5}$ & $\begin{array}{c}\beta_{5} \\
\text { (std error) }\end{array}$ & & & $\begin{array}{c}-0.025^{* *} \\
(0.012)\end{array}$ & & $\begin{array}{c}-0.025^{* *} \\
(0.012)\end{array}$ \\
\hline$K C T_{i, t-5} / K_{i, t-5}$ & $\begin{array}{c}\beta_{6} \\
\text { (std error) }\end{array}$ & & & & $\begin{array}{c}0.008 \\
(0.061)\end{array}$ & $\begin{array}{l}-0.023 \\
(0.063)\end{array}$ \\
\hline Obs. & & 364 & 364 & 355 & 364 & 355 \\
\hline Adjusted R-squares. & & 0.932 & 0.959 & 0.956 & 0.959 & 0.956 \\
\hline
\end{tabular}

(b) LSDV (least squares dummy variables, time- and country-fixed effects, robust standard errors)

\begin{tabular}{|c|c|c|c|c|c|c|}
\hline & & Model 1 & Model 2 & Model 3 & Model 4 & Model 5 \\
\hline Convergence rate & $\lambda$ & $9.6 \%$ & $13.2 \%$ & $12.6 \%$ & $13.0 \%$ & $13.0 \%$ \\
\hline $\ln \left(M P K_{i, t-5}\right)$ & $\begin{array}{c}\beta_{1} \\
\text { (std error) }\end{array}$ & $\begin{array}{c}0.619 * * * \\
(0.043)\end{array}$ & $\begin{array}{c}0.516^{* * *} \\
(0.037)\end{array}$ & $\begin{array}{c}0.533^{* * *} \\
(0.038)\end{array}$ & $\begin{array}{c}0.522^{* * *} \\
(0.040)\end{array}$ & $\begin{array}{c}0.530^{* * *} \\
(0.039)\end{array}$ \\
\hline $\ln \left(s_{i, t-5}\right)$ & $\begin{array}{c}\beta_{3} \\
\text { (std error) }\end{array}$ & & $\begin{array}{c}-0.179 * * * \\
(0.030)\end{array}$ & $\begin{array}{c}-0.152^{* * *} \\
(0.031)\end{array}$ & $\begin{array}{c}-0.176^{* * *} \\
(0.031)\end{array}$ & $\begin{array}{c}-0.154^{* * *} \\
(0.031)\end{array}$ \\
\hline $\ln \left(n_{i t}+g_{i t}+\delta\right)$ & $\begin{array}{c}\beta_{4} \\
\text { (std error) }\end{array}$ & & $\begin{array}{c}0.071^{* * *} \\
(0.027)\end{array}$ & $\begin{array}{r}0.057^{* *} \\
(0.027)\end{array}$ & $\begin{array}{c}0.071^{* * *} \\
(0.027)\end{array}$ & $\begin{array}{l}0.057^{* *} \\
(0.027)\end{array}$ \\
\hline$N_{\text {etFlow }}, t-5 / G D P_{i, t-5}$ & $\begin{array}{c}\beta_{5} \\
\text { (std error) }\end{array}$ & & & $\begin{array}{c}-0.066^{* * *} \\
(0.022)\end{array}$ & & $\begin{array}{c}-0.066^{* * *} \\
(0.023)\end{array}$ \\
\hline$K C T_{i, t-5} / K_{i, t-5}$ & $\begin{array}{c}\beta_{6} \\
\text { (std error) }\end{array}$ & & & & $\begin{array}{c}0.039 \\
(0.088)\end{array}$ & $\begin{array}{l}-0.020 \\
(0.092)\end{array}$ \\
\hline Obs. & & 364 & 364 & 355 & 364 & 355 \\
\hline Adjusted R-squares & & 0.950 & 0.963 & 0.962 & 0.963 & 0.962 \\
\hline
\end{tabular}

(c) Panel GMM (Arellano and Bond, 1991, time-fixed effects, robust standard errors)

\begin{tabular}{|c|c|c|c|c|c|c|}
\hline & & Model 1 & Model 2 & Model 3 & Model 4 & Model 5 \\
\hline Convergence rate & $\lambda$ & $3.1 \%$ & $5.6 \%$ & $7.4 \%$ & $8.3 \%$ & $8.6 \%$ \\
\hline $\ln \left(M P K_{i, t-5}\right)$ & $\begin{array}{c}\beta_{1} \\
\text { (std error) }\end{array}$ & $\begin{array}{c}0.858^{* * *} \\
(0.045)\end{array}$ & $\begin{array}{c}0.757^{* * *} \\
(0.072)\end{array}$ & $\begin{array}{c}0.690^{* * *} \\
(0.040)\end{array}$ & $\begin{array}{c}0.660^{* * *} \\
(0.045)\end{array}$ & $\begin{array}{c}0.652^{* * *} \\
(0.027)\end{array}$ \\
\hline $\ln \left(s_{i, t-5}\right)$ & $\begin{array}{c}\beta_{3} \\
\text { (std error) }\end{array}$ & & $\begin{array}{c}-0.091^{* * *} \\
(0.027)\end{array}$ & $\begin{array}{c}-0.117^{* * * *} \\
(0.018)\end{array}$ & $\begin{array}{c}-0.129^{* * *} \\
(0.021)\end{array}$ & $\begin{array}{c}-0.104^{* * *} \\
(0.012)\end{array}$ \\
\hline $\ln \left(n_{i t}+g_{i t}+\delta\right)$ & $\begin{array}{c}\beta_{4} \\
\text { (std error) }\end{array}$ & & $\begin{array}{c}0.134 * * * \\
(0.047)\end{array}$ & $\begin{array}{c}0.049 \\
(0.036)\end{array}$ & $\begin{array}{c}0.108 * * * \\
(0.027)\end{array}$ & $\begin{array}{c}0.040^{* *} \\
(0.016)\end{array}$ \\
\hline NetFlow ${ }_{i, t-5} / G D P_{i, t-5}$ & $\begin{array}{c}\beta_{5} \\
\text { (std error) }\end{array}$ & & & $\begin{array}{c}-0.084^{* * * *} \\
(0.021)\end{array}$ & & $\begin{array}{c}-0.073^{* * *} \\
(0.007)\end{array}$ \\
\hline$K C T_{i, t-5} / K_{i, t-5}$ & $\begin{array}{c}\beta_{6} \\
\text { (std error) }\end{array}$ & & & & $\begin{array}{c}-0.111^{* *} \\
(0.047)\end{array}$ & $\begin{array}{c}-0.121^{* * *} \\
(0.016)\end{array}$ \\
\hline \multicolumn{7}{|c|}{ Specification tests (p-values are in parenthesis) } \\
\hline Obs. & & 312 & 312 & 304 & 312 & 304 \\
\hline Countries & & 52 & 52 & 51 & 52 & 51 \\
\hline \# of Instruments & & 27 & 33 & 42 & 42 & 51 \\
\hline Hansen J & & $\begin{array}{c}27.29 \\
(0.127)\end{array}$ & $\begin{array}{c}26.75 \\
(0.316)\end{array}$ & $\begin{array}{c}33.93 \\
(0.375)\end{array}$ & $\begin{array}{c}32.61 \\
(0.437)\end{array}$ & $\begin{array}{c}42.77 \\
(0.353)\end{array}$ \\
\hline Diff. Hansen & & $\begin{array}{l}15.150 \\
(0.368)\end{array}$ & $\begin{array}{c}9.060 \\
(0.170)\end{array}$ & $\begin{array}{c}6.660 \\
(0.353)\end{array}$ & $\begin{array}{c}2.870 \\
(0.825)\end{array}$ & $\begin{array}{c}5.840 \\
(0.441)\end{array}$ \\
\hline $\operatorname{AR}(1)$ & & $\begin{array}{c}-3.89 \\
(0.0001)\end{array}$ & $\begin{array}{c}-3.902 \\
(0.0001)\end{array}$ & $\begin{array}{l}-3.543 \\
(0.0004)\end{array}$ & $\begin{array}{l}-3.685 \\
(0.0002)\end{array}$ & $\begin{array}{l}-3.401 \\
(0.0007)\end{array}$ \\
\hline $\mathrm{AR}(2)$ & & $\begin{array}{c}0.516 \\
(0.606)\end{array}$ & $\begin{array}{c}0.728 \\
(0.466)\end{array}$ & $\begin{array}{c}0.464 \\
(0.643)\end{array}$ & $\begin{array}{c}0.763 \\
(0.445)\end{array}$ & $\begin{array}{c}0.481 \\
(0.631)\end{array}$ \\
\hline
\end{tabular}


Table 2.3: Financial Openness and Components of Financial Flows

\begin{tabular}{|c|c|c|c|c|c|c|c|}
\hline $\begin{array}{l}\text { Financial variable } \\
\text { Period }\end{array}$ & & $\begin{array}{c}\text { Total (net, \%) } \\
(1970-2005)\end{array}$ & $\begin{array}{c}\text { CA openness } \\
(1970-2005)\end{array}$ & $\begin{array}{c}\text { FDI (net, \%) } \\
(1970-2005)\end{array}$ & $\begin{array}{c}\text { Debt (net, \%) } \\
(1970-2005)\end{array}$ & $\begin{array}{c}\text { Total (net, \%) } \\
(1970-1995)\end{array}$ & $\begin{array}{c}\text { Total (net, \%) } \\
(1985-2005)\end{array}$ \\
\hline Convergence rate & $\lambda$ & $8.6 \%$ & $3.4 \%$ & $11.2 \%$ & $8.3 \%$ & $15.2 \%$ & $8.6 \%$ \\
\hline $\ln \left(M P K_{i, t-5}\right)$ & $\begin{array}{c}\beta_{1} \\
\text { (std error) }\end{array}$ & $\begin{array}{c}0.652^{* * *} \\
(0.026)\end{array}$ & $\begin{array}{c}0.845^{* * *} \\
(0.103)\end{array}$ & $\begin{array}{c}0.570^{* * *} \\
(0.070)\end{array}$ & $\begin{array}{c}0.660^{* * *} \\
(0.032)\end{array}$ & $\begin{array}{l}0.468^{* *} \\
(0.204)\end{array}$ & $\begin{array}{c}0.652^{* * *} \\
(0.027)\end{array}$ \\
\hline $\ln \left(s_{i, t-5}\right)$ & $\begin{array}{c}\beta_{3} \\
\text { (std error) }\end{array}$ & $\begin{array}{c}-0.104^{* * *} \\
(0.012)\end{array}$ & $\begin{array}{c}-0.130^{* * * *} \\
(0.040)\end{array}$ & $\begin{array}{c}-0.143^{* * *} \\
(0.030)\end{array}$ & $\begin{array}{c}-0.133^{* * *} \\
(0.018)\end{array}$ & $\begin{array}{l}-0.055 \\
(0.097)\end{array}$ & $\begin{array}{c}-0.104^{* * *} \\
(0.012)\end{array}$ \\
\hline $\ln \left(n_{i t}+g_{i t}+\delta\right)$ & $\begin{array}{c}\beta_{4} \\
\text { (std error) }\end{array}$ & $\begin{array}{c}0.040^{* * *} \\
(0.015)\end{array}$ & $\begin{array}{c}0.148^{* *} \\
(0.064)\end{array}$ & $\begin{array}{l}0.078^{*} \\
(0.045)\end{array}$ & $\begin{array}{c}0.080^{* * *} \\
(0.017)\end{array}$ & $\begin{array}{l}0.108 \\
(0.086)\end{array}$ & $\begin{array}{c}0.040^{* *} \\
(0.016)\end{array}$ \\
\hline Financial var $_{\cdot i, t-5}$ & $\begin{array}{c}\beta_{5} \\
\text { (std error) }\end{array}$ & $\begin{array}{c}-0.073^{* * *} \\
(0.007)\end{array}$ & $\begin{array}{l}-0.027 \\
(0.164)\end{array}$ & $\begin{array}{c}-0.383^{* * * *} \\
(0.095)\end{array}$ & $\begin{array}{c}-0.044^{* * * *} \\
(0.004)\end{array}$ & $\begin{array}{c}-0.114^{* *} \\
(0.047)\end{array}$ & $\begin{array}{c}-0.073^{* * *} \\
(0.007)\end{array}$ \\
\hline$K C T_{i, t-5} / K_{i, t-5}$ & $\begin{array}{c}\beta_{6} \\
\text { (std error) }\end{array}$ & $\begin{array}{c}-0.121^{* * *} \\
(0.015)\end{array}$ & $\begin{array}{c}0.095^{* *} \\
(0.046)\end{array}$ & $\begin{array}{c}-0.271^{* * *} \\
(0.100)\end{array}$ & $\begin{array}{c}-0.182^{* * *} \\
(0.026)\end{array}$ & $\begin{array}{c}-0.382^{* *} \\
(0.156)\end{array}$ & $\begin{array}{c}-0.121^{* * *} \\
(0.016)\end{array}$ \\
\hline \multicolumn{8}{|c|}{ Specification tests (p-values are in parenthesis) } \\
\hline Obs. & & 304 & 295 & 303 & 304 & 202 & 255 \\
\hline Countries & & 51 & 51 & 51 & 51 & 51 & 51 \\
\hline \# of Instruments & & 51 & 31 & 31 & 51 & 19 & 50 \\
\hline Hansen J & & $\begin{array}{l}42.770 \\
(0.353)\end{array}$ & $\begin{array}{l}15.970 \\
(0.718)\end{array}$ & $\begin{array}{l}17.070 \\
(0.649)\end{array}$ & $\begin{array}{l}46.520 \\
(0.222)\end{array}$ & $\begin{array}{l}7.525 \\
(0.675)\end{array}$ & $\begin{array}{l}42.770 \\
(0.353)\end{array}$ \\
\hline Diff. Hansen & & $\begin{array}{l}5.840 \\
(0.441)\end{array}$ & $\begin{array}{l}5.020 \\
(0.542)\end{array}$ & $\begin{array}{l}5.330 \\
(0.502)\end{array}$ & $\begin{array}{l}4.000 \\
(0.677)\end{array}$ & $\begin{array}{l}3.090 \\
(0.543)\end{array}$ & $\begin{array}{l}5.840 \\
(0.322)\end{array}$ \\
\hline $\operatorname{AR}(1)$ & & $\begin{array}{l}-3.401 \\
(0.001)\end{array}$ & $\begin{array}{l}-3.610 \\
(0.000)\end{array}$ & $\begin{array}{l}-2.746 \\
(0.006)\end{array}$ & $\begin{array}{l}-3.398 \\
(0.001)\end{array}$ & $\begin{array}{l}-1.957 \\
(0.050)\end{array}$ & $\begin{array}{l}-3.260 \\
(0.001)\end{array}$ \\
\hline $\operatorname{AR}(2)$ & & $\begin{array}{c}0.481 \\
(0.631) \\
\end{array}$ & $\begin{array}{l}-0.644 \\
(0.520) \\
\end{array}$ & $\begin{array}{c}0.365 \\
(0.715) \\
\end{array}$ & $\begin{array}{l}0.568 \\
(0.570) \\
\end{array}$ & $\begin{array}{l}-0.364 \\
(0.716) \\
\end{array}$ & $\begin{array}{c}0.483 \\
(0.629) \\
\end{array}$ \\
\hline
\end{tabular}


Table 2.4: Various measures of trade and trade openness

\begin{tabular}{|c|c|c|c|c|}
\hline $\begin{array}{l}\text { Trade variable } \\
\text { Period }\end{array}$ & & $\begin{array}{c}K C T_{i, t-5} / K_{i, t-5} \\
(1970-2005)\end{array}$ & $\begin{array}{c}\ln \left(\mathrm{KCI}_{i, t-5}\right) \\
(1970-2005)\end{array}$ & $\begin{array}{l}\text { Trade Openness }{ }_{i, t-5} \\
\quad(1970-2005)\end{array}$ \\
\hline Convergence rate & $\lambda$ & $8.6 \%$ & $11.9 \%$ & $8.1 \%$ \\
\hline $\ln \left(M P K_{i, t-5}\right)$ & $\begin{array}{c}\beta_{1} \\
\text { (std error) }\end{array}$ & $\begin{array}{c}0.652^{* * *} \\
(0.026)\end{array}$ & $\begin{array}{c}0.552^{* * *} \\
(0.028)\end{array}$ & $\begin{array}{c}0.666^{* * *} \\
(0.029)\end{array}$ \\
\hline $\ln \left(s_{i, t-5}\right)$ & $\begin{array}{c}\beta_{3} \\
\text { (std error) }\end{array}$ & $\begin{array}{c}-0.104^{* * *} \\
(0.012)\end{array}$ & $\begin{array}{c}-0.066^{* * *} \\
(0.021)\end{array}$ & $\begin{array}{c}-0.065^{* * *} \\
(0.009)\end{array}$ \\
\hline $\ln \left(n_{i t}+g_{i t}+\delta\right)$ & $\begin{array}{c}\beta_{4} \\
\text { (std error) }\end{array}$ & $\begin{array}{c}0.040^{* * *} \\
(0.015)\end{array}$ & $\begin{array}{c}0.042^{* * *} \\
(0.009)\end{array}$ & $\begin{array}{c}0.112^{* * *} \\
(0.006)\end{array}$ \\
\hline NetFlow $_{i, t-5} / G D P_{i, t-5}$ & $\begin{array}{c}\beta_{5} \\
\text { (std error) }\end{array}$ & $\begin{array}{c}-0.073^{* * *} \\
(0.007)\end{array}$ & $\begin{array}{c}-0.130^{* * *} \\
(0.012)\end{array}$ & $\begin{array}{c}-0.087^{* * *} \\
(0.009)\end{array}$ \\
\hline Trade variable & $\begin{array}{c}\beta_{6} \\
\text { (std error) }\end{array}$ & $\begin{array}{c}-0.121^{* * *} \\
(0.015)\end{array}$ & $\begin{array}{c}-0.052^{* * *} \\
(0.011)\end{array}$ & $\begin{array}{c}-0.137^{* * *} \\
(0.013)\end{array}$ \\
\hline \multicolumn{5}{|c|}{ Specification tests (p-values are in parenthesis) } \\
\hline Obs. & & 304 & 304 & 304 \\
\hline Countries & & 51 & 51 & 51 \\
\hline \# of Instruments & & 51 & 51 & 51 \\
\hline Hansen J & & $\begin{array}{c}42.77 \\
(0.353)\end{array}$ & $\begin{array}{c}43.36 \\
(0.330)\end{array}$ & $\begin{array}{c}42.33 \\
(0.371)\end{array}$ \\
\hline Diff. Hansen & & $\begin{array}{c}5.84 \\
(0.441)\end{array}$ & $\begin{array}{c}4.52 \\
(0.607)\end{array}$ & $\begin{array}{c}7.08 \\
(0.314)\end{array}$ \\
\hline $\operatorname{AR}(1)$ & & $\begin{array}{l}-3.401 \\
(0.001)\end{array}$ & $\begin{array}{l}-3.305 \\
(0.001)\end{array}$ & $\begin{array}{l}-3.698 \\
(0.000)\end{array}$ \\
\hline $\operatorname{AR}(2)$ & & $\begin{array}{c}0.481 \\
(0.631)\end{array}$ & $\begin{array}{c}0.342 \\
(0.732)\end{array}$ & $\begin{array}{c}0.441 \\
(0.659)\end{array}$ \\
\hline
\end{tabular}


Figure 2.1: Sigma convergence of the marginal product of capital

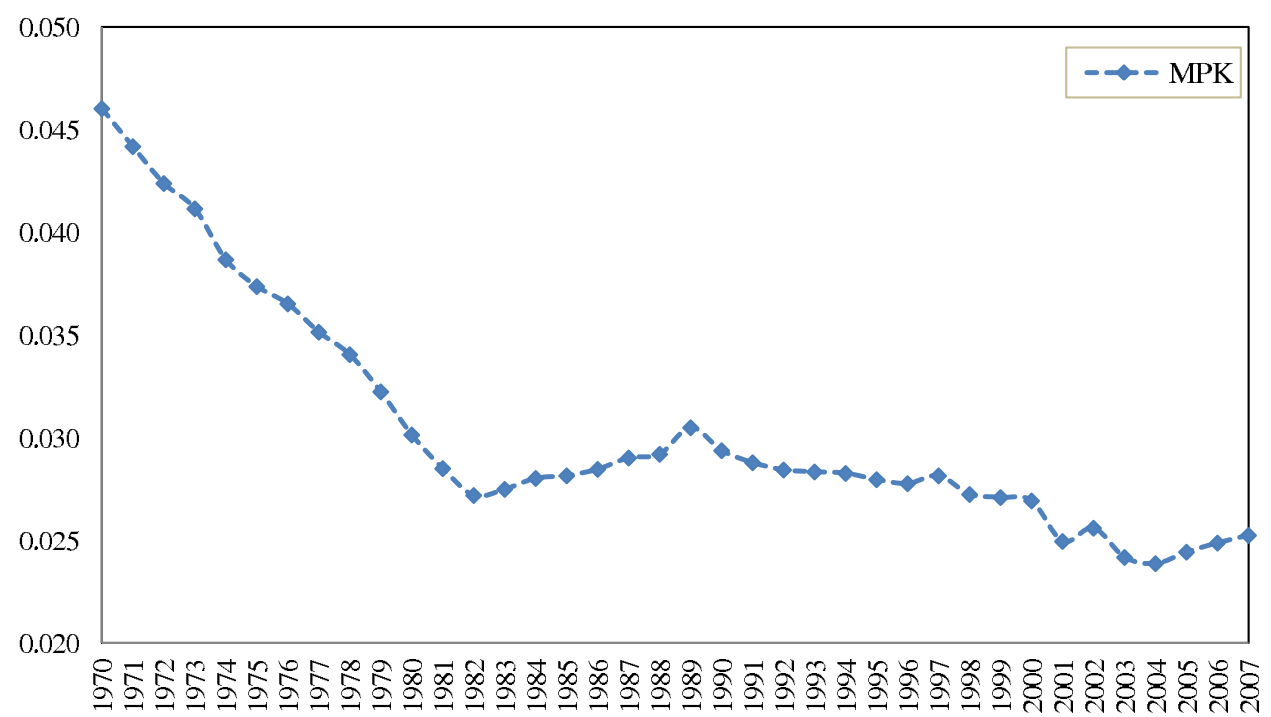

Figure 2.2: Beta convergence of the marginal product of capital

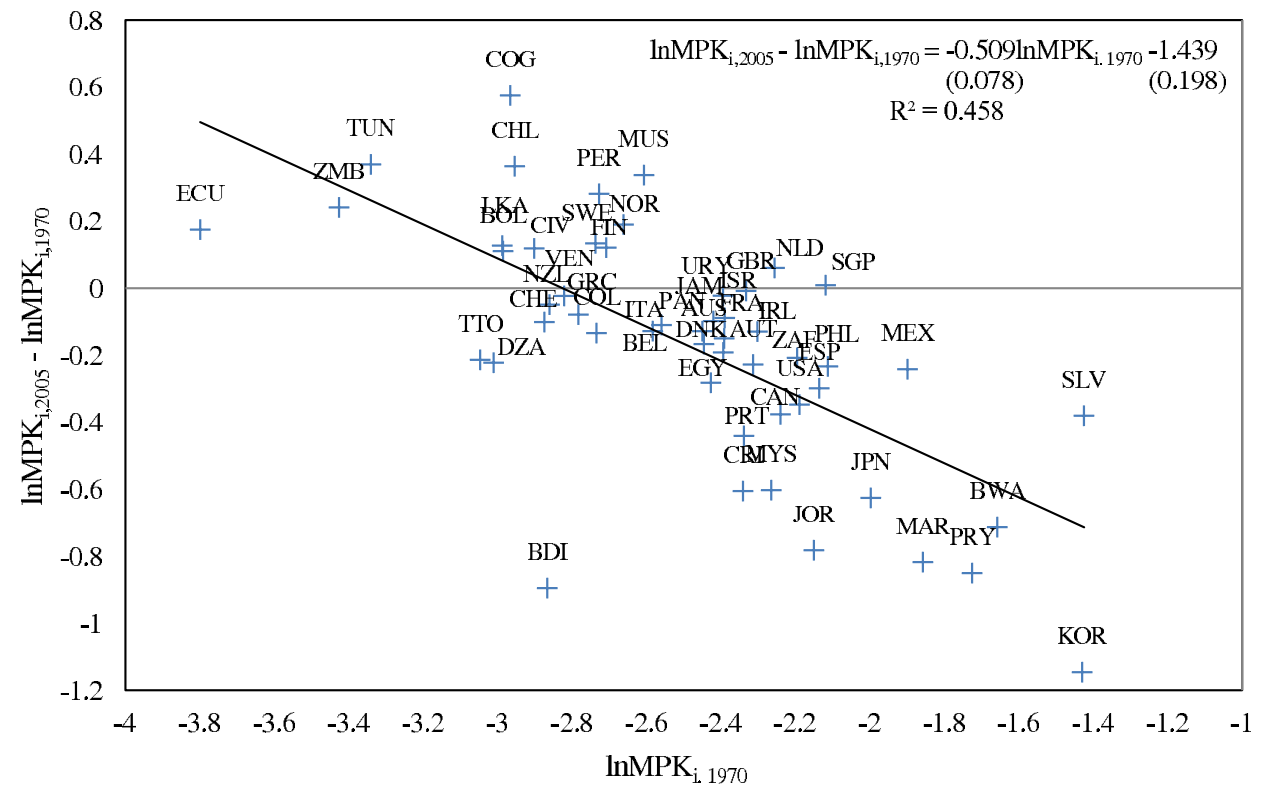


Figure 2.3: Standard deviations of the saving rates across 52 countries

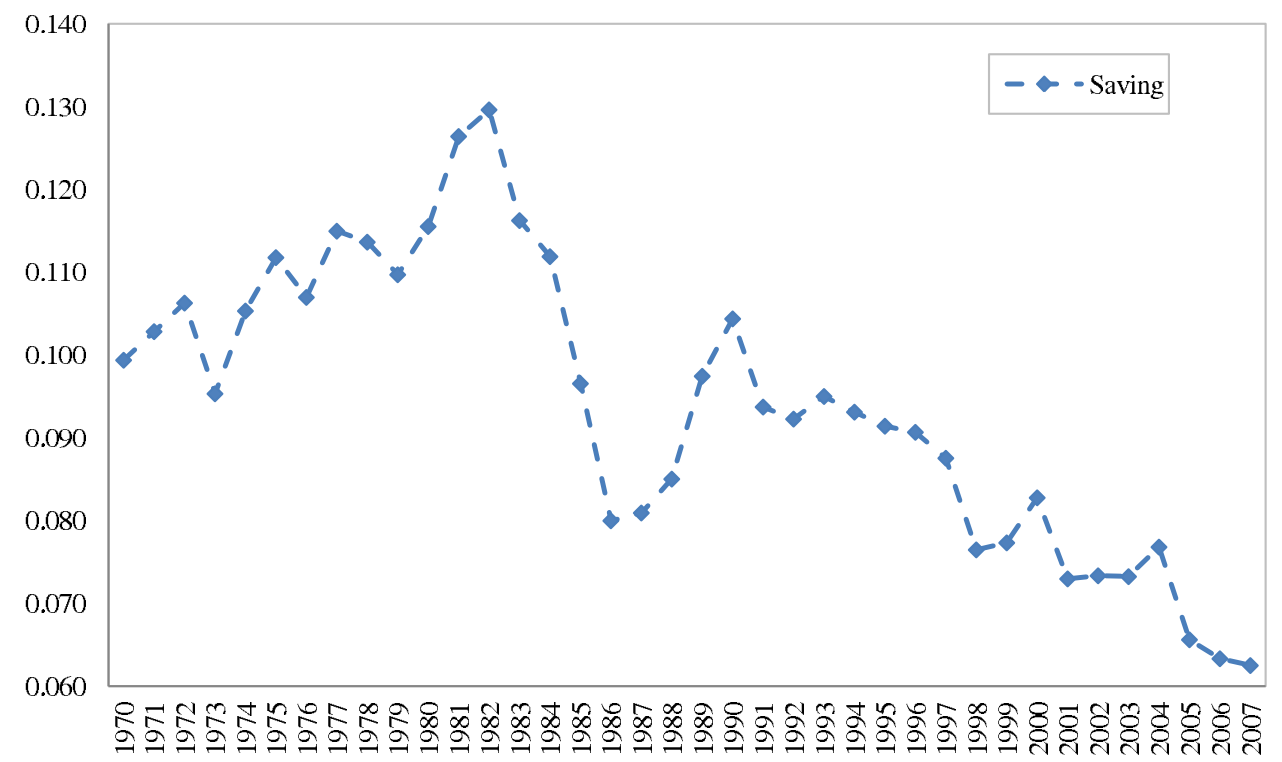

Figure 2.4: Standard deviations of the relative prices across 52 countries

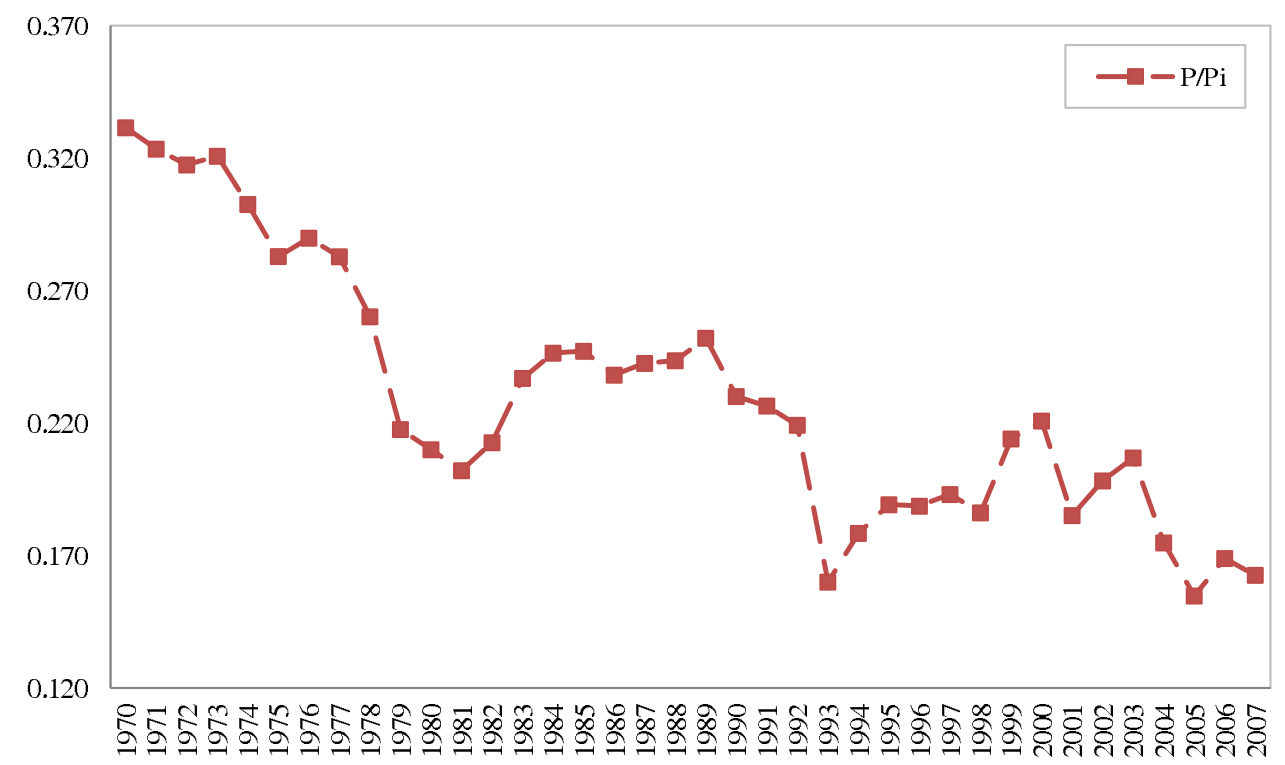


Figure 2.5: $\mathrm{MPK}_{i t}$ and $\mathrm{KCT}_{i t} / \mathrm{K}_{i t}$ in 1970

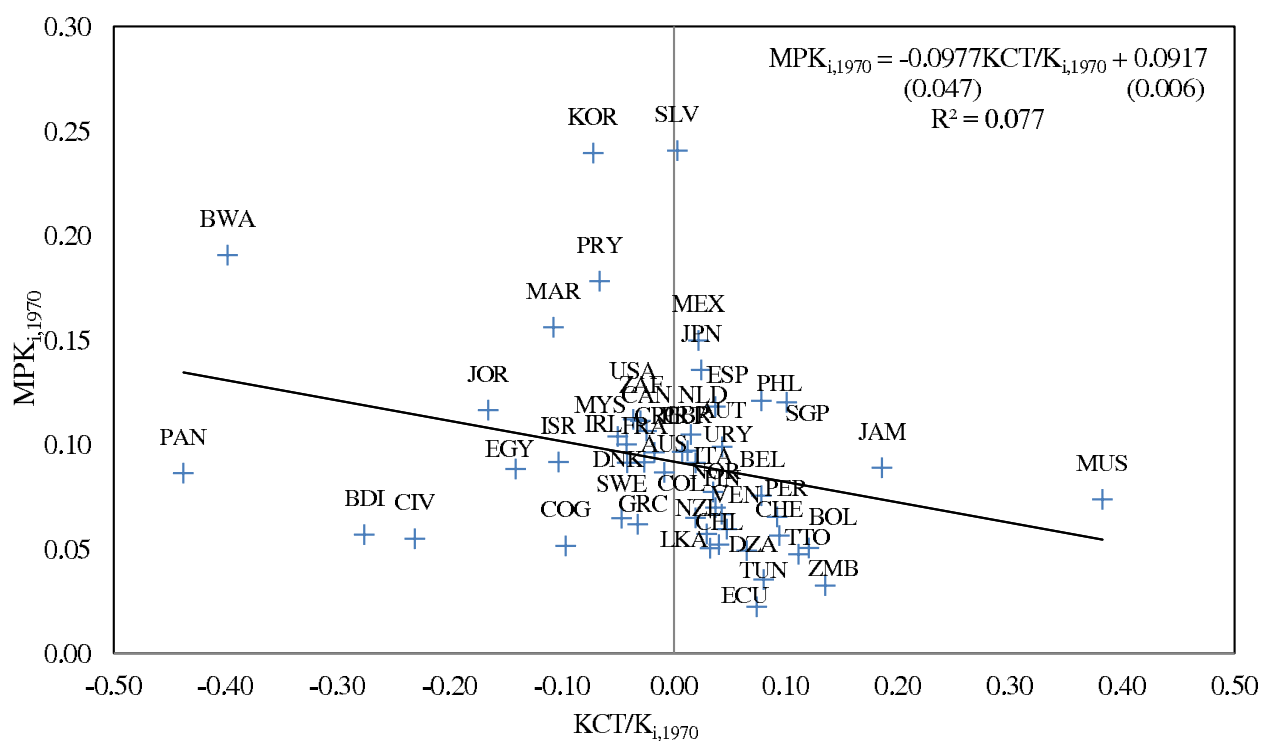

Figure 2.6: $\mathrm{MPK}_{i t}$ and $\mathrm{KCT}_{i t} / \mathrm{K}_{i t}$ in 2005

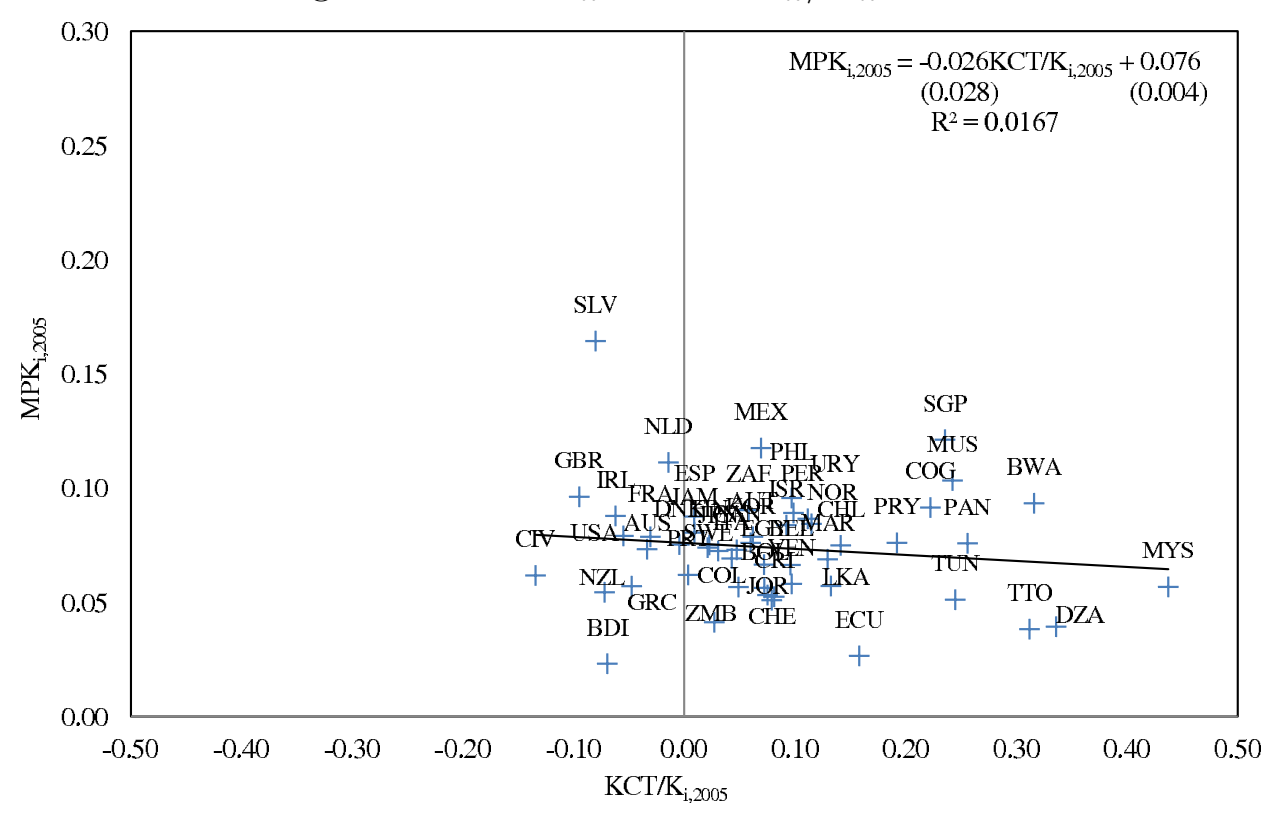




\section{Chapter 3}

\section{Capital Goods Inflows and Economic Growth}

\subsection{Introduction}

Inflows of foreign physical capital raise domestic investment and can facilitate productivity growth by means of transmission of knowledge and technological advances 1 Therefore, inflows of physical capital can increase domestic production, advance technical progress in the production of capital and speed up economic growth. Inflows can be especially beneficial for developing countries that do not raise their savings enough to increase the rate of domestic capital accumulation and have to rely on international trade to obtain physical capital. Cross-country studies on economic growth typically focus on domestic investment to explain output growth.2 However, domestic invest-

\footnotetext{
1 Coe and Helpman (1995) find that foreign R\&D capital has beneficial effects on domestic productivity. Xu and Wang (1999) show that trade in capital goods is a significant channel of R\&D spillovers. Eaton and Kortum (2001) study the effect of trade in capital goods on a country's productivity, suggesting that developing countries may benefit by importing capital-intensive goods that embody new technology.

${ }^{2}$ Empirical literature find strong empirical support for a relationship between domestic physical capital accumulation and growth in a cross-section of developing and developed countries. See for instance Mankiw et al. (1992), DeLong and Summers (1991, 1993), Barro and Sala-i-Martin (1995), Caselli et al. (1996), Jones (1997), Bernanke and Gurkavnak (2002), Bond et al. (2010) who use investment to measure capital accumulation. Baldwin (1966) is the first to point out the necessity to consider impact of capital-goods trade on the development process.
} 
ment and foreign capital inflows are tightly linked and the conventional measure of capital stock does not distinguish between domestic and foreign capital contribution. Therefore, the measure does not reflect the quality of equipment used in countries. This paper assesses a contribution of foreign capital to domestic capital investment and examines the effect of imported physical capital goods in stimulating economic growth.

I construct Solow's determinants of growth equations to study the causes of growth in real GDP per capita. The baseline equation includes domestic growth processes such as the saving rate, the population growth rate, and labor-augmenting technological progress, as discussed in Mankiw et al. (1992) and Caselli et al. (1996). Available technology and human capital determines the ability to 'absorb' new capital stock and benefit from technology transfers (Eaton and Kortum, 2001). The benefits from physical capital inflows may be small in a country with underdeveloped production infrastructure or with a poorly educated work force. Domestic factors also control for the differences in steady states of different countries. To consider an open economy version, I augment the model with inflows of foreign capital goods as a share of domestic investment. Foreign capital includes machinery, transport equipment and other production capital.

To empirically examine the contribution of foreign capital goods to economic growth, I employ ordinary least squares (OLS) and least squares dummy variable (LSDV) estimators, similar to Mankiw et al. (1992) and Islam (1995). Then, I introduce the dynamic panel model and follow the empirical methodology proposed by Caselli et al. (1996) and supported by Shioji (2001) and Bond et al. (2010). They use a difference General Methods of Moments estimator (GMM) introduced by Arellano and Bond (1991). The estimation procedure involves first-differencing of the growth regression that removes country-specific effects, and therefore, the omitted variable bias. Then, right-hand side variables are instrumented with their lagged values that solves endogeneity issue. I employ non-overlapping 5-year period data that allows the reduction 
of the impact of the business cycle fluctuations or occasional shocks, as well as measurement errors 3 I develop a data set of 52 countries for the period from 1970 to 2005, which includes 24 high-income (OECD) and 28 low to middle income (non-OECD) countries.

The empirical evidence suggests that a higher share of foreign physical capital in domestic investment predicts a higher level of output per capita growth. Non-industrial (non-OECD) countries that rely on imported foreign capital grow faster. Imports of capital goods contribute to the economic development by providing production opportunities and ground for innovations and technological advance. A challenge for policymakers in developing countries is then to prepare their economies to best absorb the potential benefits of physical capital inflows in order to generate long-term domestic benefits, and facilitate trade in capital goods. Industrial (OECD) countries can also benefit from the trade in capital goods given the cross-country differences in a price of capital.

The remainder of this paper is organized as follows. Section 3.2 presents specifications of the neoclassical growth model augmented with international capital. Section 3.3 describes the data, the properties of the main variables and discusses a trend in capital goods trade. Section 3.4 outlines the econometric approach, validity tests and presents empirical results. Section 3.5 concludes.

\subsection{Neoclassical Growth Model}

Following the discussion and notation in Mankiw et al. (1992), I consider the neoclassical Cobb-Douglas production function that exhibits constant returns to scale, positive and diminishing returns to private inputs, and satisfies the Inada conditions:

$$
Y_{i t}=K_{i t}^{\alpha}\left(A_{i t} L_{i t}\right)^{(1-\alpha)}
$$

\footnotetext{
${ }^{3}$ See Bond et al. (2001) and Bond et al. (2010) for details.
} 
where $Y_{i t}$ is output in country $i$ at the time $t, K_{i t}$ capital, $L_{i t}$ labor, $A_{i t}$ is the level of technology, and $\alpha$ is a constant with $0<\alpha<1$. Labor force and technology growth rates are assumed to be $n$ and $g$ :

$$
\begin{aligned}
& L_{i t}=L_{0} e^{n t} \\
& A_{i t}=A_{0} e^{g t}
\end{aligned}
$$

Let $s_{i t}$ be the fraction of output that is saved, that is the savings rate. Savings are invested into physical capital stock, that depreciates at the constant rate $\delta>0$. Let $\mathrm{k}$ be the stock of capital per effective unit of labor, $k_{i t}=K_{i t} / A_{i t} L_{i t}$, and y be the level of output per effective unit of labor, $y_{i t}=Y_{i t} / A_{i t} L_{i t}$. Then the net increase in the stock of physical capital at a point in time equals gross investment less depreciation given available level of technology and labor:

$$
\frac{\partial k_{i t}}{\partial t}=I_{i t}-\delta k_{i t}=s_{i t} y_{i t}-\left(n_{i t}+g_{i t}+\delta\right) k_{i t}
$$

where the term $\left(n_{i t}+g_{i t}+\delta\right)$ can be thought of as the effective depreciation rate for the capital-labor ratio, $k_{i t}=K_{i t} / A_{i t} L_{i t}$. This equation defines the evolution of the capital stock per effective unit of labor and implies that $k_{i t}$ converges to a steady-state value:

$$
k_{i t}^{*}=\left[\frac{s_{i t}}{\left(n_{i t}+g_{i t}+\delta\right)}\right]^{\frac{1}{1-\alpha}}
$$

This fundamental equation developed by Solow (1956) and Swan (1956) implies that the steady-state capital-labor ratio is related positively to the savings rate and negatively to the effective depreciation rate. To obtain the effect of the savings rate and the effective depreciation rate on the growth of per capita GDP, the steady-state condition is substituted into the production function and logs are taken. The following equation represents the steady-state output per capita condition:

$$
\ln \left[\frac{Y_{i t}}{A_{i t} L_{i t}}\right]=\ln A_{0}+g t+\frac{\alpha}{1-\alpha} \ln \left(s_{i t}\right)-\frac{\alpha}{1-\alpha} \ln \left(n_{i t}+g_{i t}+\delta\right)
$$


This model implies that the growth in output per effective labor is positively associated with the savings rate and negatively with population growth, the depreciation rate and technology attainment. The $A_{0}$ term reflects country-specific levels of technology, and consists of a constant and a country-specific shock. The model defines the determinants of economic development level in a closed version economy.

\subsubsection{Growth Regression in a Closed Economy}

I aim to empirically examine economic factors that cause the growth in real GDP per worker. First, I consider country domestic factors that play fundamental roles in the growth process. The growth of output per worker is determined by the savings rate and effective depreciation rate:

$$
\ln \left(\frac{Y_{i, t}}{A_{i t} L_{i t}}\right)=\beta_{1} \ln \left(s_{i, t}\right)+\beta_{2} \ln \left(n_{i t}+g_{i t}+\delta\right)+\xi_{t}+\eta_{i}+\epsilon_{i t}
$$

where $\xi_{t}$ represents the time trend, $\eta_{i}$ account for the country-specific intercept or unobserved factors, such as differences in aggregate production functions. It can also define climate, institutions and resources $4 \epsilon_{i t}$ is an error term. The equation predicts that real income growth is higher in countries with a higher savings rate $\left(s_{i, t}\right)$, and lower in countries with a lower effective depreciation rate $\left(n_{i t}+g_{i t}+\delta\right)$.

I relax the theoretical assumption that the included explanatory variables, the savings rate and the effective depreciation rate, are independent of country-specific factors affecting the production function. I assume instead that domestic factors correlate with country-specific factors, that is they are not independent of $\eta_{i}$. This creates omitted variable bias and estimates obtained with ordinary least squared (OLS) may be biased.

\footnotetext{
${ }^{4}$ This country-specific effect correlates with the included explanatory variables that creates omitted variable bias (Islam, 1995).
} 


\subsubsection{Augmenting the Solow Model with Inflows of Physical Capital}

International trade in capital goods is one of the significant sources for capital accumulation and productivity growth. To account for the effect of international trade in capital goods on output growth, I augment the Solow model with the shares of foreign physical capital goods in domestic investment. This way, I obtain the composition of new investment which represents foreign capital, knowledge and technology. The data on international capital goods trade is obtained from UN Comtrade database. The trade is comprised of the commodities in SITC section 7: nonelectrical machinery, electrical machinery, apparatus, appliances, and transport equipment. I used a measure of gross capital formation as a proxy for domestic investment 5 Accessing the share of foreign capital goods in domestic investment allows to distinguish the quality of production capital, that is to determine to what extent a country relies on imports of foreign capital.

The main model is augmented to include the shares of foreign production capital in gross domestic investments, $\left(K \operatorname{Imp} / I_{i, t}\right)$ :

$$
\ln \left(Y_{i, t}\right)=\beta_{1} \ln \left(s_{i, t}\right)+\beta_{2} \ln \left(n_{i t}+g_{i t}+\delta_{i t}\right)+\beta_{3}(K \operatorname{Imp} / I)_{i, t}+\xi_{t}+\eta_{i}+\epsilon_{i t}
$$

I expect a positive relationship between the share of foreign capital goods in domestic investment and economic growth. This would imply that the higher the share of imported equipment in domestic capital formation leads to the higher growth in real output per worker. Figures (3.1) and (3.2) depict a scatterplot with the share of capital imports in domestic investment and real GDP per worker in 1970 and 2005. The figures show that while some countries import most of their equipment, the majority

\footnotetext{
${ }^{5}$ I use nominal values (\$US) for manufactures exports, imports, and gross capital formation to measure capital trade as a share of domestic investment, as well as net manufactures trade as a share of domestic investment. The data on gross domestic formation is obtained from WDI.
} 
of countries rely on domestic producers. This evidence can be due to the existence of large trade costs and due to the fact that the reported price of equipment is actually higher in richer countries (Eaton and Kortum, 2001; Hsieh and Klenow, 2007). The investment share of foreign equipment varies little across countries 6 Figures display a small, but positive relationship between the share of foreign capital goods in investment and a country's development level.

Figure (3.3) plots the share of capital goods exports from the 'Big 6' over the period from 1970 to 2005. The group of countries include Canada, France, Japan, Italy, the United Kingdom and the United States. These capital-intensive countries specialize in producing and exporting capital goods. The scatterplot shows that the share of capital goods exports from the 'Big 6' is about 80\% of the total capital exports in 1970 and declines over time. It reaches $61 \%$ in 2005. This evidence implies that while big industrial countries continute to be major suppliers of capital goods, non-industrial countries may rely less and less on imported capital goods.

\subsection{Data}

The data set contains 52 countries 7 over a period from 1970 to 2005 . The data on $Y_{i t}, s_{i t}, n_{i t}$ comes from the Penn World Table 7.0 (Heston et al., 2011). $Y_{i t}$ is real GDP in constant 2005 international dollars. The saving rate, $s_{i t}$, is defined as the share of

\footnotetext{
Eaton and Kortum (2001) find that the investment share that is applied to equipment does not vary much across countries compared to the equipment production share. They argue that if equipment investment shares vary a lot, then there would not be a positive relationship between equipment production and net exports of equipment.

${ }^{7} 52$ countries are Algeria (DZA), Australia (AUS), Austria (AUT), Belgium (BEL), Bolivia (BOL), Botswana (BWA), Burundi (BDI), Canada (CAN), Chile (CHL), Colombia (COL), Congo (COG), Costa Rica (CRI), Cote d'Ivoire (CIV), Denmark (DNK), Ecuador (ECU), Egypt (EGY), El Salvador (SLV), Finland (FIN), France (FRA), Greece (GRC), Ireland (IRL), Israel (ISR), Italy (ITA), Jamaica (JAM), Japan (JPN), Jordan (JOR), Korea (KOR), Malaysia (MYS), Mauritius (MUS), Mexico (MEX), Morocco (MAR), the Netherlands (NLD), New Zealand (NZL), Norway (NOR), Panama (PAN), Paraguay (PRY), Peru (PER), Philippines (PHL), Portugal (PRT), Singapore (SGP), South Africa (ZAF), Spain (ESP), Sri Lanka (LKA), Sweden (SWE), Switzerland (CHE), Trinidad \&Tobago (TTO), Tunisia (TUN), the United Kingdom (GBR), the United States (USA), Uruguay (URY), Venezuela (VEN), and Zambia (ZMB).
} 
real domestic investment to real GDP. The depreciation rate of physical capital, $\delta$, is assumed to be $6 \%$ and constant for all countries in all years. The average population growth rate, $n_{i t}$, is calculated over $t-\tau$ and $t$. The growth rate of labor productivity, $g_{i t}$, is calculated from the fitted values of $\left(\bar{A}_{i t}\right)$ from the following equation over $t-\tau$ to $t$ :

$$
\ln \left(A_{i t}\right)=\lambda_{i}^{1} \ln \left(E d u_{i t}^{1}\right)+\lambda_{i}^{2} \ln \left(E d u_{i t}^{2}\right)+\lambda_{i}^{3} \ln \left(E d u_{i t}^{3}\right)+\lambda_{t}^{4}+\varepsilon_{i t}
$$

where $E d u_{i t}^{1}, E d u_{i t}^{2}$, and $E d u_{i t}^{3}$ are the average years of primary schooling, secondary schooling, and tertiary schooling, respectively, from Barro and Lee (2010).

The data set is an unbalanced panel consisting of about 416 observations at 5-year intervals $(\tau=5)$. Table 3.1 displays some basic statistics about output per capita and physical capita trade variables in 1970 and 2005. In 1970, the lowest and highest values of real GDP per capita and its growth were in Burundi (\$US 354.47) and in Switzerland (\$US 25940.07), correspondingly. This shows that Switzerland is about 73 times richer than Burundi and the growth of its real GDP per capita is twice as high compared to Burundi's. In 2005, the country with highest real GDP per capita is Norway (\$US 48701.210), while Burundi obtains the lowest value (\$US 368.536) again. There is a high dispersion in income levels due to the insignificant growth of some low-income countries, which increases over the studied period. Norway is approximately 132 times richer than Burundi in 2005 in terms of per capita GDP.

Capital trade variables are shares of capital imports in domestic investment, shares of capital exports in total exports and net trade in capital goods (as a share of domestic investment). Japan obtains the lowest level of manufactures imports as a share of domestic investment in 1970 and 2005. Uruguay and Singapore import the highest amount of capital goods in 1970 and 2005, correspondingly. Ecuador and Panama have the lowest shares of capital exports in total exports, while the United States maintain 
the highest share of capital exports across all countries in 1970 and 2005. Finally, net capital goods trade numbers confirm that net-importers of foreign capital are developing countries (Uruguay and Burundi), and capital producers and net-exports are developed countries (United Kingdom and Singapore).

\subsection{Empirical Evidence}

To study economic growth, I employ baseline equation (3.1) and extend it with the share of imported capital in investment. First, I estimate a closed economy version of the Solow model that includes only domestic factors, denoted as Model 1. Next, Model 2 adds the share of foreign capital in investment, as shown in the equation (3.2). Estimations are performed for three country groups: OECD, non-OECD and for all countries. I estimate the models by ordinary least squares (OLS) estimator with time dummy variables and then by least squares dummy variables (LSDV) estimator including time- and country-specific dummy variables. Although both estimators are biased 8 , the results provide the rough ranges of the true parameters. Model 1 also serve as benchmark model, and I compare its results with prominent results obtained by Mankiw et al. (1992) and Islam (1995).

Next, I modify the baseline equation to include the past values of the dependent variable, similar to Caselli et al. (1996); Shioji (2001); Bond et al. (2010). The proper estimator for the dynamic panel model specification is a two-step GMM estimator. The estimator is asymptotically efficient and robust to panel-specific autocorrelation, different patterns of heteroskedasticity, and cross-country correlation (Bond et al., 2001). It is a proper estimator for a short time-span $(T=8)$ and a large cross-section $(N=52$

\footnotetext{
ECaselli et al. (1996), Shioji (2001) and Bond et al. (2010) argue that the existing empirical literature on economic convergence produces inconsistent and unreliable estimations. Two sources of inconsistency are stressed. First is omitted variable bias due to mistreatment of country-specific fixed effects. Seconds is endogeneity of explanatory variables due to the dynamic nature of the model. Existing growth literature assumes that country effects are uncorrelated with other right-hand-side variables and do not typically solve engodeneity issues. This paper attempts to address these concerns.
} 
for full sample, $N=24$ for OECD and $N=28$ for non-OECD samples ). Further, to prevent any sort of cross-country and contemporaneous correlations, I remove universal time-related shocks from errors by including time dummy variables.

Estimation results from OLS are reported in Table 3.2(a), In Model 1, the coefficients on the savings rate $\left(s_{i, t-\tau}\right)$ are positive and statistically significant in non-OECD countries and in the full sample. The coefficients on the effective depreciation rate $\left(n_{i t}+g_{i t}+\delta\right)$ are negative and statistically significant in the OECD countries and the full sample. The coefficients have opposite effects in sign that signals that the jointhypothesis of the Solow model is confirmed and the aggregate production function is Cobb-Douglas. The estimates are similar to those obtained by Mankiw et al. (1992). In Models 2, the share of foreign capital appears positively related to output growth. A $1 \%$ increase in the share leads to a $0.201 \%$ increase in income per effective labor in OECD countries and a $0.337 \%$ increase in non-OECD countries. This result suggests that developing countries that rely on the imported capital goods benefit more compared to the developed countries in terms of output per worker growth. However, the estimates are biased due to endogeneity and omitted variable issues.

Next, I estimate the three models with LSDV, including time and country-specific dummy variables. The estimator corrects for individual effects bias, but does not solve the endogeneity issue. The results are reported in Table 3.2(b). Here, the coefficients on saving rate $\left(\beta_{2}\right)$ and effective depreciation rate $\left(\beta_{3}\right)$ are opposite in sign across all models. Compared to the corresponding OLS coefficients, domestic factors a exhibit weaker and insignificant relationship with output growth in non-OECD countries. This bias may be due to the fact that country-specific dummy variables may capture cross-country variation in the relatively stable effective depreciation rate. Overall, the estimation results for Model 1 are consistent with those obtained by Islam (1995). The coefficients on the share of foreign capital in Model 2 are positive and statistically significant at the $1 \%$ confidence level for non-OECD countries and the full sample. A $1 \%$ increase in the share of foreign capital in investment leads to an approximate a $0.2 \%$ 
increase in output per effective labor. The coefficient on the share of foreign capital in investment appears to be negative in OECD countries. While the results are prone to endogeneity bias, the estimator significantly improves the goodness of fit of the model, raising the adjusted R-squared from 0.063 to 0.965 in the Model 2 of the full sample.

Further, I consider the dynamic model specification. The difference GMM transforms equation (3.2) into a first-difference equation:

$$
\begin{aligned}
\Delta \ln \left(Y_{i, t}\right)= & \beta_{1} \Delta \ln \left(Y_{i, t-5}\right)+\beta_{2} \Delta \ln \left(s_{i, t}\right)+\beta_{3} \Delta \ln \left(n_{i t}+g_{i t}++\delta_{i t}\right)+ \\
& \left.+\beta_{4} \Delta\left(K_{i m p} / I\right)_{i, t}+\delta\right)+\xi_{t}+\Delta \epsilon_{i t}
\end{aligned}
$$

where $\xi_{t}$ is a year-specific constant, capturing factors common to all countries. Firstdifference transformation removes country-specific fixed effects. I treat domestic factors in the equation above as exogenous and lagged dependent variable along with the foreign factor as endogenous variables. Lagged levels of endogenous regressors are used as instruments. The validity of instruments is determined by the consistency of parameter estimates and specification tests: the Hansen test for overidentifying restrictions, difference-in-Hansen tests for exogeneity of instrument subsets, and the Arellano-Bond (AR) test for first- and second-order serial correlation in differences. These specification tests indicate that the moment conditions are robust. The test for second-order correlation in differences, $\operatorname{AR}(2)$ is important because it detects autocorrelation in levels.

The results of GMM estimation and its specification tests are reported in Table 3.3. The Hansen and difference-Hansen statistics signal that errors are not serially correlated. I find no evidence for serial correlation in levels, suggesting that fluctuations in economic activity do not influence the results. The coefficient on lagged output has the expected positive sign, and it strongly significant. The coefficients on $\ln (s)$ and $\ln \left(n_{i t}+g_{i t}+\delta\right)$ are opposite in sign and statistically significant in most models. A closed 
version of the neoclassical model shows that the savings rate is positively associated with the growth in output per worker, while population growth together with the depreciation rate and the level of education attainment, have negative effect on output per worker.

Model 2 indicates that physical capital inflows have positive and significant effects on output growth in developing countries. A $1 \%$ increase in the share of foreign capital goods in the domestic investment is associated with a $0.08 \%$ increase in output per worker. The relationship between the two measures appear to be positive, however insignificant and smaller in OECD countries compared to non-OECD countries. A 1 $\%$ increase in the share of foreign capital in investment leads to a $0.037 \%$ increase in income per effective labor. This is not a surprising result given that developed countries are large producers of capital goods and have less need to import.

The effect of domestic savings on output growth is slightly higher in industrial countries relative to non-industrial. A $1 \%$ increase in the savings rate leads to a $0.419 \%$ increase in output per worker in developed countries and a $0.409 \%$ increase in developing countries. Across all countries, the effect of the foreign capital share in the domestic investment is relatively similar to the effect of domestic savings on output growth. While a $1 \%$ increase in the share of capital leads to a $0.176 \%$ increase in output per worker, a $1 \%$ increase in domestic savings leads to a $0.197 \%$ increase in output per worker.

Overall, across all countries, the empirical evidence suggests that an increase in the share of foreign physical capital in domestic investment facilitates positive growth in output per effective labor. Imported capital contributes significantly to the domestic capital investment in developing countries. This conclusion, however, does not necessary imply that we would observe an increase in imports of foreign capital goods as country's domestic investment rises, meaning that a nation industry can not easily switch from producing consumption goods to capital goods 9

\footnotetext{
${ }^{9}$ To determine whether foreign capital goods and domestic investment are indeed substitutes and to
} 


\subsection{Conclusion}

Trade in physical capital is a conduit for technological advances and innovations transfers, that have a potential to stimulate economic growth. This paper examines the relationship between the share of foreign capital in domestic investment and the growth of real gross domestic products per worker across 22 OECD and 28 non-OECD countries. I use the data on bilateral trade in manufactures goods from UN Comtrade to obtain the data on trade in physical capital. The data represents non-overlapping fiveyear periods from 1970 to 2005. I augment Solow's neoclassical growth model to include international inflows of physical capital to study the determinants of growth in output per capita. I employ OLS, LSDV, and the two-step difference GMM estimators and find that the savings rate and the share of foreign capital in investment are significant factors of real GDP per worker growth.

The results predict that the role of foreign capital in explaining per capita output growth is higher in non-industrial countries (non-OECD). Investment in nonindustrial countries is constrained by the lack of domestic capital, therefore countries rely on manufacturing imports to obtain physical capital. Imports of capital goods contribute to the economic development by providing production opportunities, ground for innovations and technological advance. Policies should create the incentives for productive physical capital accumulation through international trade in capital, as well as stimulate technological advance and human capital growth in order to develop the appropriate capacity to absorb advanced technology from abroad. The results are robust to alternative specifications, controlling for country-specific effects and time trends.

calculate the elasticity of substitution, a constant elasticity of substitution (CES) production function is needed. 


\subsection{Tables and Figures}

Table 3.1: Summary Statistics

\begin{tabular}{|c|c|c|c|c|}
\hline & Mean & Std. Dev. & Min & $\operatorname{Max}$ \\
\hline \multicolumn{5}{|l|}{ Year: 1970} \\
\hline Real GDP per capita $(P P P, 2005=100)$ & 22104.13 & 15288.58 & 698.567 ( Burundi) & 51363.61 (Switzerland) \\
\hline Real GDP per capita growth (\%) & 9.655 & 0.968 & 6.549 (Burundi) & 10.846 (Switzerland) \\
\hline Capital Goods Imports (\%, Investment) & 0.296 & 0.182 & 0.026 (Japan) & 1.005 (Uruguay) \\
\hline Capital Goods Exports (\%, Total Exports) & 2.272 & 5.185 & 0.00004 (Ecuador) & 28.340 (United States) \\
\hline Net Capital Goods Trade (\%, Investment) & -0.209 & 0.200 & -0.983 (Uruguay) & 0.173 (United Kingdom) \\
\hline \multicolumn{5}{|l|}{ Year: 2005} \\
\hline Real GDP per capita $(P P P, 2005=100)$ & 38143.62 & 27757.8 & 703.813 (Burundi) & 93836.13 (Norway) \\
\hline Real GDP per capita growth (\%) & 10.156 & 1.045 & 6.556 (Burundi) & 11.449 (Norway) \\
\hline Capital Goods Imports $(\%, \mathrm{I})$ & 0.595 & 0.658 & 0.120 (Japan) & 4.464 (Singapore) \\
\hline Capital Goods Exports (\%, Total Exports) & 2 & 3.851 & 0.00003 (Panama) & 19.055 (United States) \\
\hline Net Capital Goods Trade (\%, Investment) & -0.175 & 0.285 & -1.002 (Burundi) & 0.926 (Singapore) \\
\hline
\end{tabular}

Table 3.2: Economic Growth and Physical Capital Inflows: OLS and LSDV

(a) OLS (ordinary least squares, time-fixed effects, robust standard errors)

\begin{tabular}{lccccccc}
\hline & & \multicolumn{2}{c}{ OECD } & \multicolumn{2}{c}{ Non-OECD } & \multicolumn{2}{c}{ Full Sample } \\
\cline { 2 - 7 } Dependent variable: $\ln \left(Y_{i, t}\right)$ & Model 1 & Model 2 & Model 1 & Model 2 & Model 1 & Model 2 \\
\hline $\ln \left(s_{i, t}\right)$ & $\beta_{1}$ & 0.034 & 0.211 & $0.700^{* * *}$ & $0.543^{* * *}$ & $0.741^{* * *}$ & $0.585^{* * *}$ \\
& (std error) & $(0.133)$ & $(0.139)$ & $(0.110)$ & $(0.115)$ & $(0.132)$ & $(0.153)$ \\
$\ln \left(n_{i t}+g_{i t}+\delta\right)$ & $\beta_{2}$ & $-0.721^{* * *}$ & $-0.477^{* *}$ & -0.224 & -0.029 & $-1.093^{* * *}$ & $-0.830^{* * *}$ \\
& (std error) & $(0.225)$ & $(0.216)$ & $(0.222)$ & $(0.268)$ & $(0.231)$ & $(0.273)$ \\
$(K I m p / I)_{i, t}$ & $\beta_{3}$ & & $0.201^{* *}$ & & $0.337^{* * *}$ & & 0.110 \\
& (std error) & & $(0.089)$ & & $(0.114)$ & & $(0.139)$ \\
Constant & & $8.807^{* * *}$ & $9.612^{* * *}$ & $9.586^{* * *}$ & $9.723^{* * *}$ & $8.325^{* * *}$ & $8.766^{* * *}$ \\
& & $(0.499)$ & $(0.460)$ & $(0.507)$ & $(0.665)$ & $(0.526)$ & $(0.684)$ \\
& & & & & & & \\
Obs. & 192 & 184 & 224 & 188 & 416 & 372 \\
Adjusted R-sq. & & 0.285 & 0.256 & 0.180 & 0.182 & 0.116 & 0.063 \\
\hline
\end{tabular}

(b) LSDV (least squares dummy variables, time- and country-fixed effects, robust standard errors)

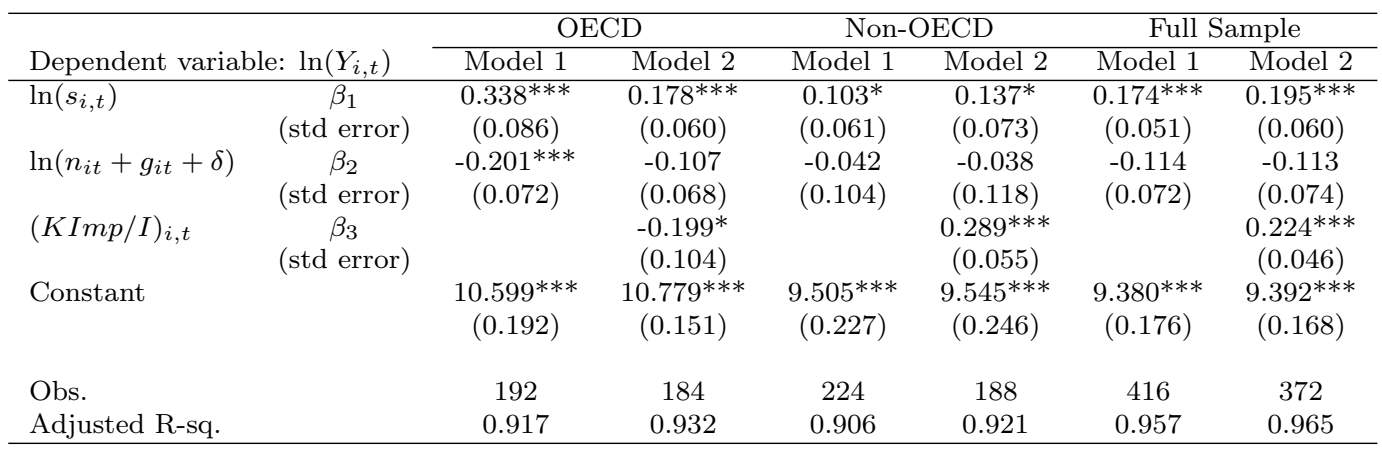


Table 3.3: Economic Growth and Physical Capital Inflows: GMM Panel GMM (Arellano and Bond, 1991, time-fixed effects, robust standard errors)

\begin{tabular}{|c|c|c|c|c|c|c|}
\hline \multirow[b]{2}{*}{ Dependent variable: $\ln \left(Y_{i, t}\right)$} & \multicolumn{2}{|c|}{ OECD } & \multicolumn{2}{|c|}{ Non-OECD } & \multicolumn{2}{|c|}{ Full Sample } \\
\hline & Model 1 & Model 2 & Model 1 & Model 2 & Model 1 & Model 2 \\
\hline $\begin{array}{c}\beta_{1} \\
\text { (std error) }\end{array}$ & $\begin{array}{c}0.732^{* * *} \\
(0.033)\end{array}$ & $\begin{array}{c}0.627^{* * *} \\
(0.075)\end{array}$ & $\begin{array}{c}0.775^{* * *} \\
(0.059)\end{array}$ & $\begin{array}{c}0.615^{* * *} \\
(0.049)\end{array}$ & $\begin{array}{c}0.495^{* * *} \\
(0.091)\end{array}$ & $\begin{array}{c}0.320^{* * *} \\
(0.018)\end{array}$ \\
\hline $\begin{array}{c}\beta_{2} \\
\text { (std error) }\end{array}$ & $\begin{array}{c}0.419^{* * *} \\
(0.033)\end{array}$ & $\begin{array}{c}0.409^{* * *} \\
(0.023)\end{array}$ & $\begin{array}{c}0.226^{* * *} \\
(0.071)\end{array}$ & $\begin{array}{c}0.134^{* * *} \\
(0.038)\end{array}$ & $\begin{array}{c}0.142^{* * *} \\
(0.040)\end{array}$ & $\begin{array}{c}0.197 * * * \\
(0.013)\end{array}$ \\
\hline $\begin{array}{cc}\ln \left(n_{i, t} t+g_{i, t}+?_{i, t}\right) & \beta_{3} \\
(\text { Kimp } / I)_{i, t} & (\text { std error) } \\
\beta_{4} & (\text { std error })\end{array}$ & $\begin{array}{c}-0.184^{* * *} \\
(0.026)\end{array}$ & $\begin{array}{c}-0.140 * * * \\
(0.026) \\
0.037 \\
(0.066)\end{array}$ & $\begin{array}{c}0.020 \\
(0.028)\end{array}$ & $\begin{array}{c}-0.035 \\
(0.034) \\
0.088^{* * *} \\
(0.017)\end{array}$ & $\begin{array}{c}-0.069 * * * \\
(0.026)\end{array}$ & $\begin{array}{c}-0.057^{* * *} \\
(0.011) \\
0.176^{* * *} \\
(0.012)\end{array}$ \\
\hline \multicolumn{7}{|c|}{ Specification tests (p-values are in parenthesis) } \\
\hline Obs. & 144 & 138 & 168 & 132 & 312 & 270 \\
\hline Countries & 24 & 23 & 28 & 28 & 52 & 51 \\
\hline \# of Instruments & 19 & 20 & 19 & 28 & 23 & 44 \\
\hline Hansen $\mathrm{J}$ & $\begin{array}{c}13.68 \\
(0.188)\end{array}$ & $\begin{array}{c}10.65 \\
(0.386)\end{array}$ & $\begin{array}{c}11.78 \\
(0.300)\end{array}$ & $\begin{array}{c}20.02 \\
(0.332)\end{array}$ & $\begin{array}{c}16.07 \\
(0.309)\end{array}$ & $\begin{array}{c}30.93 \\
(0.619)\end{array}$ \\
\hline Diff. Hansen & $\begin{array}{c}11.57 \\
(0.172)\end{array}$ & $\begin{array}{c}7.87 \\
(0.446)\end{array}$ & $\begin{array}{c}10.03 \\
(0.263)\end{array}$ & $\begin{array}{c}5.96 \\
(0.652)\end{array}$ & $\begin{array}{c}8.47 \\
(0.389)\end{array}$ & $\begin{array}{c}7.95 \\
(0.439)\end{array}$ \\
\hline $\operatorname{AR}(1)$ & $\begin{array}{c}-2.53 \\
(0.011)\end{array}$ & $\begin{array}{c}-1.58 \\
(0.115)\end{array}$ & $\begin{array}{c}-2.44 \\
(0.015)\end{array}$ & $\begin{array}{c}-1.56 \\
(0.119)\end{array}$ & $\begin{array}{c}-2.05 \\
(0.040)\end{array}$ & $\begin{array}{c}-0.60 \\
(0.546)\end{array}$ \\
\hline $\operatorname{AR}(2)$ & $\begin{array}{c}0.56 \\
(0.574)\end{array}$ & $\begin{array}{c}0.00 \\
(0.996)\end{array}$ & $\begin{array}{c}-0.16 \\
(0.873)\end{array}$ & $\begin{array}{c}0.11 \\
(0.910)\end{array}$ & $\begin{array}{c}0.15 \\
(0.878)\end{array}$ & $\begin{array}{c}0.71 \\
(0.476)\end{array}$ \\
\hline
\end{tabular}


Figure 3.1: Real GDP per worker and Capital Goods Imports in 1975

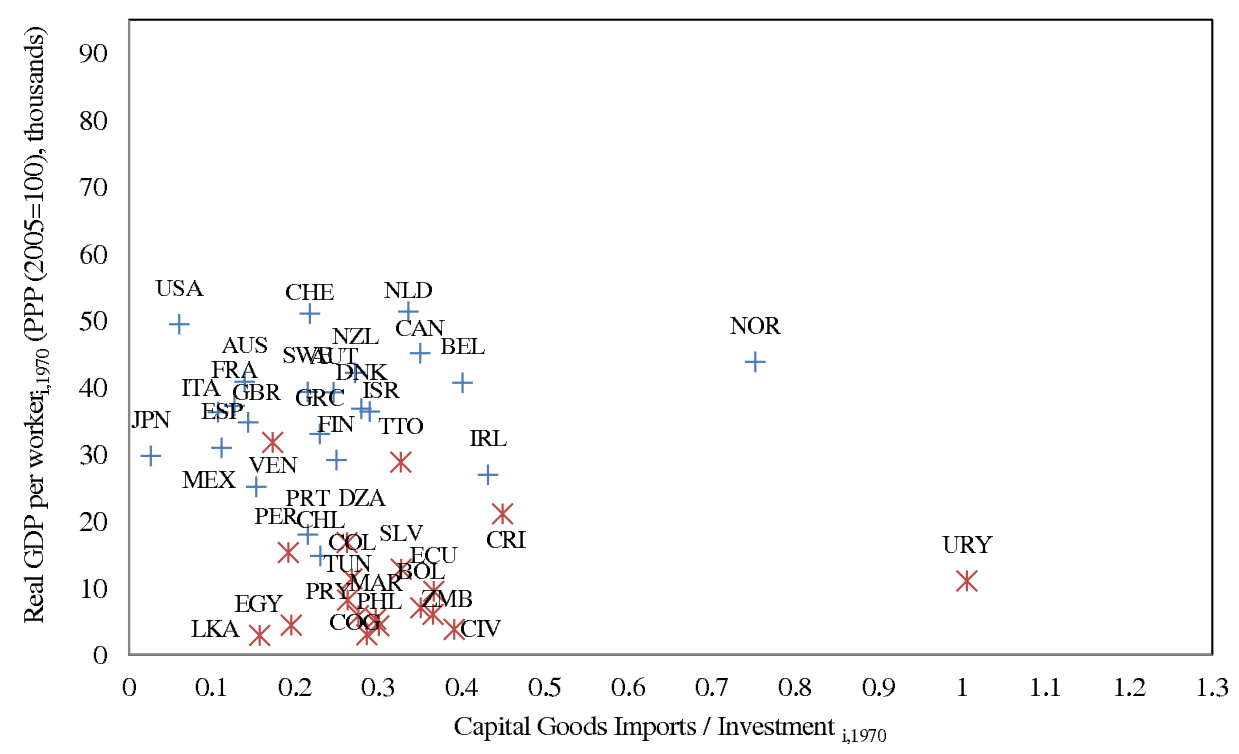

Figure 3.2: Real GDP per worker and Capital Goods Imports in 2005

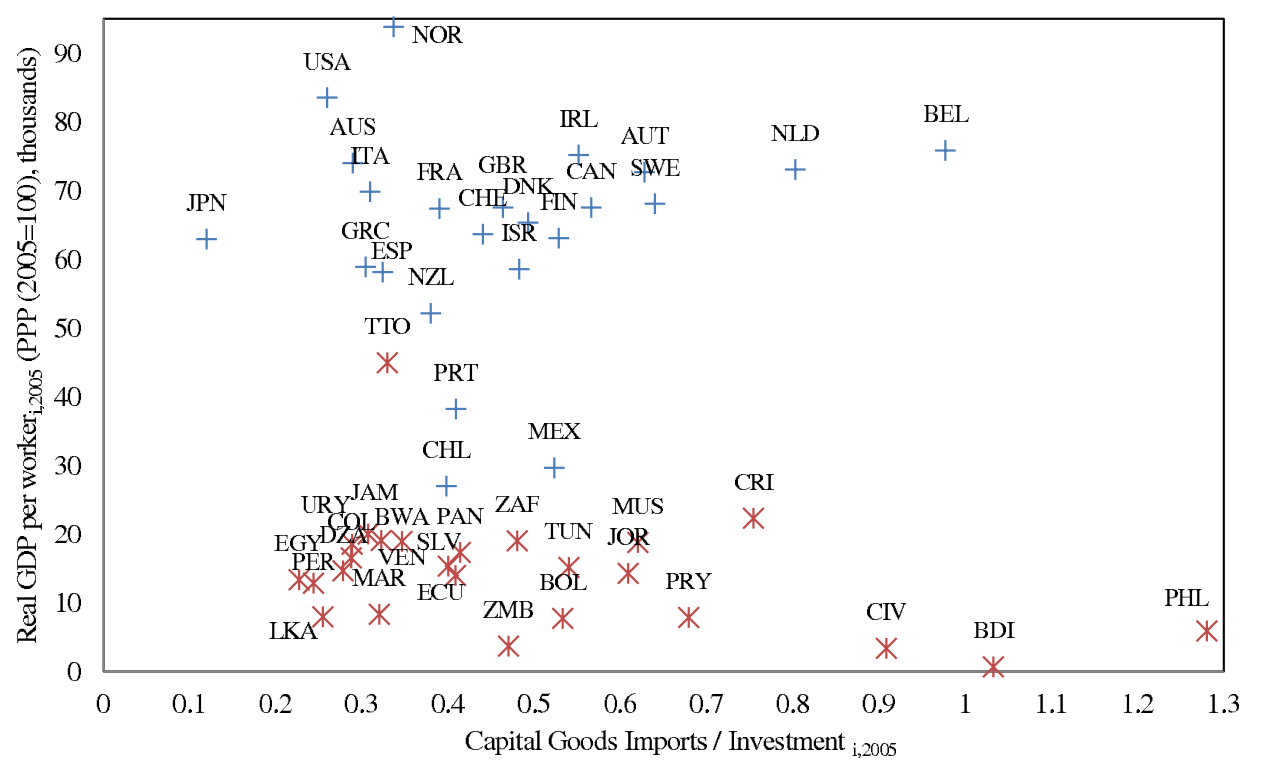


Figure 3.3: Share of Capital Goods Export in Total Capital Exports, G6 countries

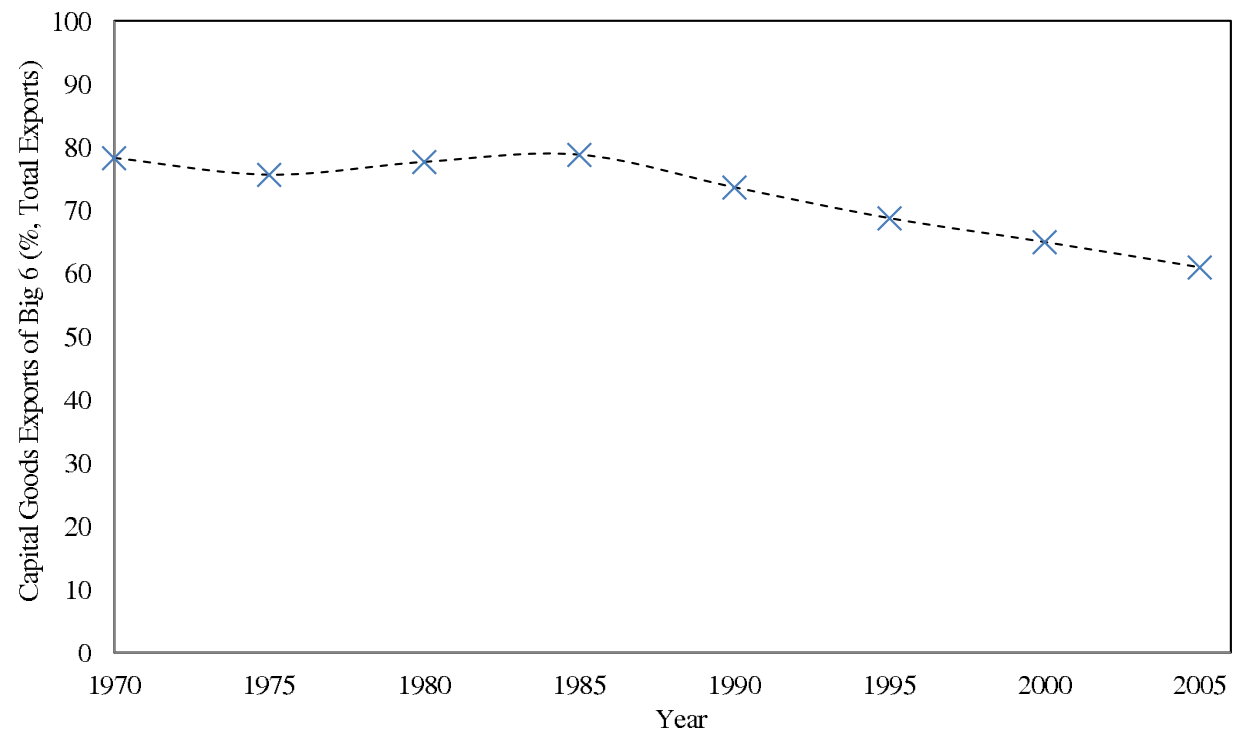




\section{References}

P. Aghion, P. Bacchetta, R. Ranciere, and K. Rogoff. Exchange rate volatility and productivity growth: The role of financial development. Journal of Monetary Economics, 56:494-513, 2009.

J. Aizenman. On the hidden links between financial and trade opening. Journal of International Money and Finance, 27:372-386, 2008.

J. Aizenman and I. Noy. Fdi and trade-two way linkages? The Quarterly Review of Economics and Finance, 46(3):317-337, 2006.

J. Aizenman and I. Noy. Endogenous financial and trade openness. Review of Development Economics, 13(2):175-189, 2009.

P. Antràs and R. J. Caballero. Trade and capital flows: A financial frictions perspective. Journal of Political Economy, 117(4):701-744, 2009.

M. Arellano and S. Bond. Some tests of specification for panel data: Monte carlo evidence and an application to employment equations. Review of Economic Studies, 58(2):277-97, 1991.

R. Baldwin. The role of capital-goods trade in the theory of international trade. American Economic Review, 56(4):841-848, September 1966.

R. J. Barro and J. Lee. A new data set of educational attainment in the world, 1950- 
2010. NBER Working Papers 15902, National Bureau of Economic Research, Inc, 2010 .

R. J. Barro and X. Sala-i-Martin. Convergence. The Journal of Political Economy, 100 (2):223-251, 1992.

R. J. Barro and X. Sala-i-Martin. Economic growth. New York: McGraw-Hill, 1995.

B. S. Bernanke and R. S. Gurkaynak. Is growth exogenous? taking mankiw, romer, and weil seriously. In NBER Macroeconomics Annual 2001, Volume 16, NBER Chapters, pages 11-72. National Bureau of Economic Research, Inc, 2002.

A. S. Blinder. Wage discrimination: Reduced form and structural estimates. Journal of Human Resources, 8:436-455, 1973.

B. Blonigen. In search of substitution between foreign production and exports. Journal of International Economics, 53(1):81-104, 2001.

S. Bond, A. Hoeffler, and J. Temple. Gmm estimation of empirical growth models. Economics Papers 2001-W21, Economics Group, Nuffield College, University of Oxford, 2001.

S. Bond, A. Leblebicioglu, and F. Schiantarelli. Capital accumulation and growth: a new look at the empirical evidence. Journal of Applied Econometrics, 25(7):10731099, 2010.

C. Bush and E. Hanschel. The effectiveness of capital controls - the case of slovenia. Journal of Economic Integration, 15(4):601-628, 2000.

G. Caprio, P. Honohan, and J. Stiglitz. Financial Liberalization: how far, how fast? Cambridge University Press, Cambridge, UK, 2001.

M. Cardenas and F. Barrera. On the effectiveness of capital controls: The experience of colombia during the 1990s. Journal of Development Economics, 54:27-57, 1997. 
F. Caselli and J. Feyrer. The marginal product of capital. The Quarterly Journal of Economics, 122(2):535-568, 2007.

F. Caselli, G. Esquivel, and F. Lefort. Reopening the convergence debate: A new look at cross-country growth empirics. Journal of Economic Growth, 1(3):363-89, 1996.

S. Chatterjee and K. Naknoi. The marginal product of capital, capital flows and convergence. The American Economic Review, 100(2):73-77, 2010.

M. Chinn and H. Ito. What matters for financial development? capital controls, institutions, and interactions. Journal of Development Economics, 81:163-192, 2006.

M. Chinn and H. Ito. A new measure of financial openness. Journal of Comparative Policy Analysis: Research and Practice, 10(3):309 - 322, 2008.

D. Coe and E. Helpman. International r\&d spillovers. European Economic Review, 39 (5):859-887, May 1995.

D. R. Davis and D. E. Weinstein. An account of global factor trade. American Economic Review, 91(5):1423-1453, 2001.

G. Dell'Ariccia, J. di Giovanni, A. Faria, A. Kose, J. Ostry, M. Schindler, P. Mauro, and M. Terrones. Reaping the benefits of financial globalization. International Monetary Fund Occasional Paper 264, 2007.

J. B. DeLong and L. H. Summers. Equipment investment and economic growth. The Quarterly Journal of Economics, 106(2):445-502, May 1991.

J. B. DeLong and L. H. Summers. How strongly do developing economies benefit from equipment investment? Journal of Monetary Economics, 32(3):395-415, December 1993.

J. Eaton and S. Kortum. Trade in capital goods. European Economic Review, 45(7): 1195-1235, 2001. 
S. Edwards. Sequencing of reforms, financial globalization, and macroeconomic vulnerability. Journal of The Japanese and International Economies, 23:131-148, 2009.

M. Feldstein and C. Horioka. Domestic saving and international capital flows. The Economic Journal, 67(268):pp. 591-624, 1980.

J. Frankel and A. Rose. Currency crisis in emerging markets: Empirical indicators. Journal of International Economics, 41:351-68, 1996.

A. Heston, R. Summers, and B. Aten. Penn world table version 7.0. Technical report, Center for International Comparisons of Production, Income and Prices at the University of Pennsylvania, 2011.

C-T. Hsieh and P. Klenow. Relative prices and relative prosperity. The American Economic Review, 97(3):562-585, 2007.

IMF. Globalization and inequality. In World Economic Outlook. IMF, 2007.

N. Islam. Growth empirics: A panel data approach. The Quarterly Journal of Economics, 110(4):pp. 1127-1170, 1995.

H. Ito. Financial development and financial liberalization in asia: Thresholds, institutions and the sequence of liberalization. The North American Journal of Economics and Finance, 17(3):303-327, 2006.

B. Johnston and N. Tamirisa. Why do countries use capital controls? IMF Working Paper, International Monetary Fund, 98(181):1-37, 1998.

C. I. Jones. Convergence revisited. Journal of Economic Growth, 2(2):131-53, July 1997.

A. Kose, E. Prasad, K. Rogoff, and S. Wei. Financial globalization: A reappraisal. IMF Staff Papers, International Monetary Fund, 56(1):8-62, 2009. 
P. Lane and G. Milesi-Ferretti. The external wealth of national mark II: Revisited and extended estimates of foreign assets and liabilities. Journal of International Economics, 73(69):223250, 2007.

M. Leahy. Contributions of financial systems to growth in oecd countries. OECD Economic Department Working Paper 280, 2001.

E. E. Leamer. What's the use of factor contents? Journal of International Economics, 50:17-49, 2000 .

D. Leblang. Domestic and systemic determinants of capital controls in the developed and developing world. International Studies Quarterly, pages 435-454, 1997.

F. R. Lichtenberg and B. v. Pottelsberghe de la Potterie. International r\&d spillovers: A comment. European Economic Review, 42(8):1483-1491, 1998.

N. Loayza, K. Schmidt-Hebbel, and L. Serven. Saving in developing countries: An overview. The World Bank Economic Review, 14(3):393-414, 2000.

R. Lucas. Why doesn't capital flow from rich to poor countries? The American Economic Review, 80(2):92-96, 1990.

G. Mankiw, D. Romer, and D. Weil. A contribution to the empirics of economic growth. The Quarterly Journal of Economics, 107(2):pp. 407-437, 1992.

X. Sala-i-Martin. Regional cohesion: Evidence and theories of regional growth and convergence. European Economic Review, 40(6):1325-1352, 1996.

M. Mello. Estimates of the marginal product of capital, 1970-2000. The B.E. Journal of Macroeconomics, 9(1):1-28, 2009.

R. Mundell. International trade and factor mobility. The American Economic Review, 47(3):321-335, 1957. 
S. Nishioka. International differences in production techniques: Implications for the factor content of trade. forthcoming at Journal of International Economics, 2012.

R. L. Oaxaca. Male-female wage differentials in urban labor markets. International Economic Review, 14:693-709, 1973.

OECD. Forty years' experience with the oecd code of liberalization of capital movements. summary and conclusions. OECD: Directorate For Financial, Fiscal and Enterprise Affairs, 2002.

R. Rajan. International financial liberalization in developing countries: Lessons from recent experience. Center for International Economic Studies, Discussion Paper 0220, 2002. http://www.adelaide.edu.au/cies/papers/0220.pdf.

P. Samuelson. International factor-price equalization once again. The Economic Journal, 59(234), 1949.

E. Shioji. Public capital and economic growth: A convergence approach. Journal of Economic Growth, 6, 2001.

R. Solow. A contribution to the theory of economic growth. The Quarterly Journal of Economics, 70(1), 1956.

R. Solow. Technical change and the aggregate production function. The Review of Economics and Statistics, 39(3), 1957.

J. Stiglitz. Capital market liberalization and exchange rate regimes: Risk without reward. Annals of the American Academy of Political and Social Science, 579:219$248,2002$.

W. F. Stolper and P. A. Samuelson. Protection and real wages. Review of Economic Studies, 9(1):pp. 5873, 1941. 
T. Swan. Economic growth and capital accumulation. Economic Record, 32:334-361, 1956.

J. Vanek. The factor proportions theory: The n-factor case. Kyklos, 21:749-756, 1968.

S. Vlades-Prieto and S. Soto. The effectiveness of capital controls: Theory and evidence from chile. Empirica, 25:133-164, 1998.

WDI. World development indicators, the world bank, 2007.

B. Xu and J. Wang. Capital goods trade and r\&d spillovers in the oecd. Canadian Journal of Economics, 32(5):1258-1274, 1999. 